\title{
Organic superbase $t$-Bu-P4 catalyzes amination of methoxy(hetero)arenes
}

Masanori Shigeno, ${ }^{*}$ Kazutoshi Hayashi, Kanako Nozawa-Kumada, and Yoshinori Kondo*

Department of Biophysical Chemistry, Graduate School of Pharmaceutical Science,

Tohoku University, Aoba, Sendai, 980-8578, Japan

*E-mail: mshigeno@m.tohoku.ac.jp, ykondo@m.tohoku.ac.jp, Fax: (+81) 22-795-6804.

Supporting Information

Table of Contents

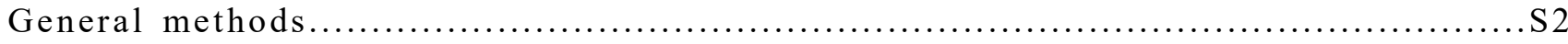

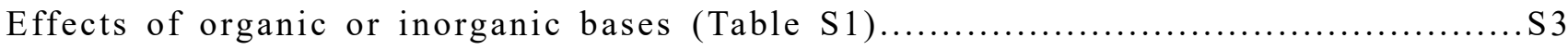

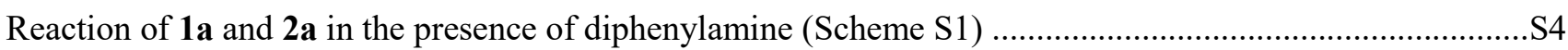

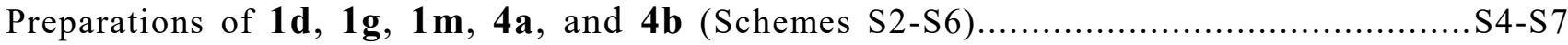

Experimental procedures and spectra data for obtained products.................................................S7-S19

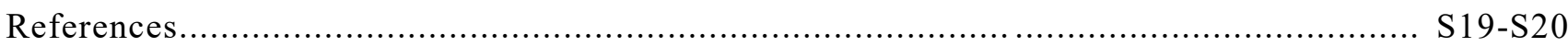

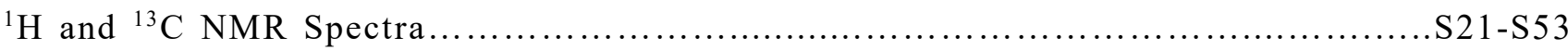


General methods. All reactions were carried out under $\mathrm{N}_{2}$ or Ar atmosphere. Flash column chromatography was performed with Kanto silica gel $60 \mathrm{~N}$ (spherical, neutral, 70-230 $\mu \mathrm{m}$ ). Preparative thin-layer chromatography was performed with silica gel (Wakogel ${ }^{\circledR}$ B-5F). Melting points (Mp) were determined with a Yazawa micro melting point apparatus without correction. Infrared (IR) data were recorded on SensIR ATR (Attenuated Total Reflectance) FT-IR, and absorbance frequencies are reported in reciprocal centimeters $\left(\mathrm{cm}^{-1}\right)$. NMR data were recorded on a JEOL AL400 spectrometer (395.75 MHz for ${ }^{1} \mathrm{H}, 99.50 \mathrm{MHz}$ for ${ }^{13} \mathrm{C}$ ). Chemical shifts are expressed in $\delta$ (parts per million, ppm) values, and coupling constants are expressed in herts $(\mathrm{Hz}) .{ }^{1} \mathrm{H}$ NMR spectra were referenced to tetramethylsilane as an internal standard or to a solvent signal $\left(\mathrm{CDCl}_{3}: 7.26 \mathrm{ppm}\right) .{ }^{13} \mathrm{C}$ NMR spectra were referenced to a solvent signal $\left(\mathrm{CDCl}_{3}: 77.0 \mathrm{ppm}\right)$. Low and high resolution mass spectra (LRMS and HRMS) were obtained from Mass Spectrometry Resource, Graduate School of Pharmaceutical Sciences, Tohoku University, on a JEOL JMS-DX 303 and JMS-700/JMS-T 100 GC spectrometer, respectively. Gel permeation chromatography (GPC) was conducted with a Recycling Preparative HPLC LC-9210 (Japan Analytical Industry, Co. Ltd.). 
Table S1. Effects of organic or inorganic bases ${ }^{a}$

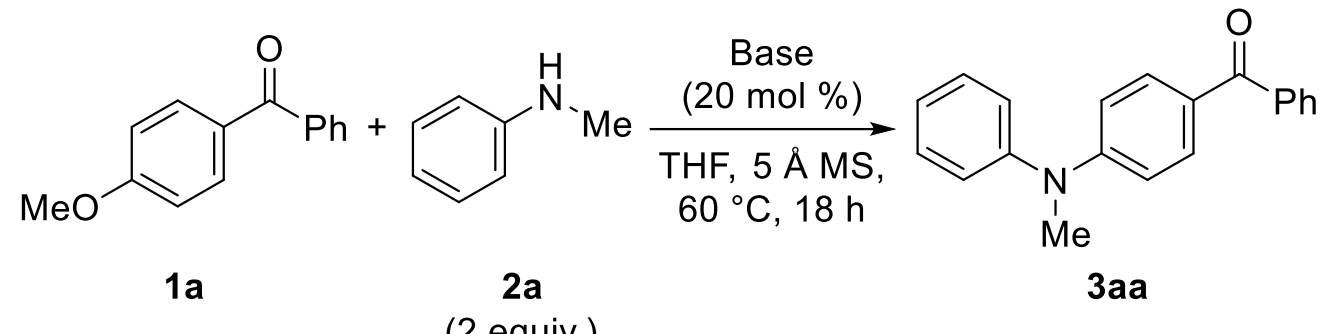

( 2 equiv.)

\begin{tabular}{|c|c|c|}
\hline Entry & Base & 3aa $(\%)^{b}$ \\
\hline 1 & $\mathrm{NEt}_{3}$ & 0 \\
\hline 2 & $i-\operatorname{Pr}_{2} \mathrm{NEt}$ & 0 \\
\hline 3 & Pyridine & 0 \\
\hline 4 & Proton sponge ${ }^{c}$ & 0 \\
\hline 5 & $\mathrm{DBU}^{d}$ & 0 \\
\hline 6 & $\mathrm{TBD}^{e}$ & 0 \\
\hline 7 & $t-\mathrm{Bu}-\mathrm{P} 2^{f}$ & 0 \\
\hline 8 & $\mathrm{NaO}-t-\mathrm{Bu}$ & 0 \\
\hline 9 & $\mathrm{KO}-t-\mathrm{Bu}$ & 2 \\
\hline 10 & $\mathrm{NaH}$ & 2 \\
\hline 11 & LiHMDS $^{g}$ & 0 \\
\hline 12 & $\mathrm{NaHMDS}^{g}$ & 5 \\
\hline 13 & KHMDS $^{g}$ & 14 \\
\hline
\end{tabular}

${ }^{a} 1 \mathrm{a}(0.20 \mathrm{mmol}), \mathbf{2 a}(0.40 \mathrm{mmol})$, base $(0.04 \mathrm{mmol}), 5 \AA \mathrm{MS}(100 \mathrm{mg}), \mathrm{THF}(0.7 \mathrm{~mL}), 60{ }^{\circ} \mathrm{C}, 18 \mathrm{~h}$. ${ }^{b}$ The yields were determined by ${ }^{1} \mathrm{H}-\mathrm{NMR}$ using 1,1,2-trichloroethane as an internal standard. ${ }^{c}$ Proton sponge: 1,8-bis-(dimethylamino)naphthalene. ${ }^{d} \mathrm{DBU}$ : 1,8-diazabicyclo[5.4.0]-7-undecene. ${ }^{e} \mathrm{TBD}$ : 1,5,7-triazabicyclo-[4.4.0]dec-5-ene. $\quad f_{t \text {-Bu-P2: }}$ 1-(tert-butylimino)-1,1,3,3,3-

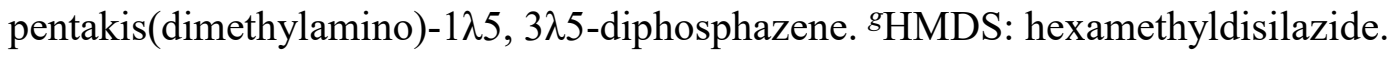


Scheme S1. Reaction of $1 \mathbf{a}$ and $\mathbf{2 a}$ in the presence of diphenylamine ${ }^{a}$

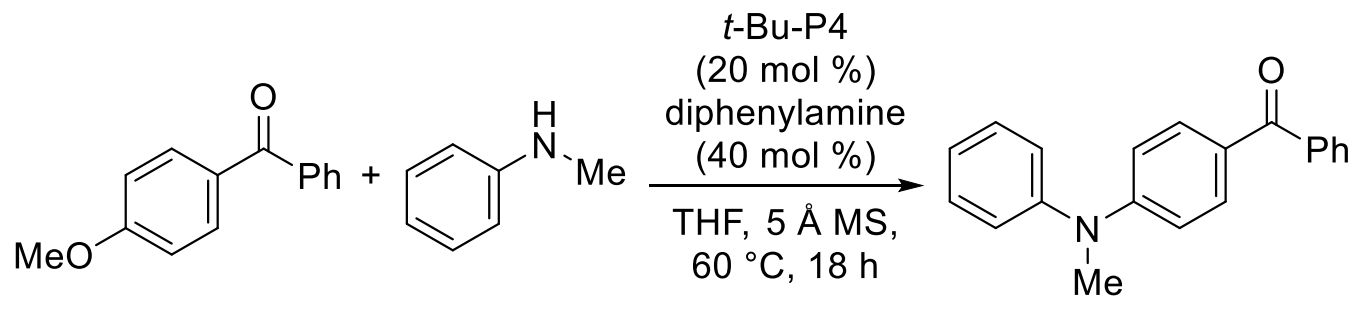

1a

2a

3 aa, $2 \%^{b}$

(2 equiv.)

${ }^{a} 1 \mathrm{a}(0.20 \mathrm{mmol}), 2 \mathrm{a}(0.40 \mathrm{mmol}), t$-Bu-P4 (0.04 mmol), diphenylamine (0.08 mmol), $5 \AA \mathrm{MS}(100 \mathrm{mg}), \mathrm{THF}$ $(0.7 \mathrm{~mL}), 60{ }^{\circ} \mathrm{C}$, and $18 \mathrm{~h} .{ }^{b}$ The yield was determined by ${ }^{1} \mathrm{H}-\mathrm{NMR}$ using 1,1,2-trichloroethane as an internal standard.

Materials. Unless otherwise noted, materials were purchased from Tokyo Kasei Co., Aldrich Inc. and other commercial suppliers and were used as received. $\mathbf{1 a}^{1}, \mathbf{1 e}^{2}, \mathbf{1 f}^{1}, \mathbf{1 h}^{3}, \mathbf{1 k}^{4}, \mathbf{1 l}^{5}, \mathbf{1 n}^{6}, \mathbf{S 5} \mathbf{7}^{7}$, and $\mathbf{S 7 ^ { 1 }}$ were prepared according to the literature procedures. $\mathbf{1 d}, \mathbf{1 g}, \mathbf{1} \mathbf{m}, \mathbf{4 a}$, and $\mathbf{4 b}$ were prepared according to the following Schemes S2-S6. Flash column chromatography was performed with Kanto silica gel $60 \mathrm{~N}$ (spherical, neutral, 70-230 mesh).

Scheme S2. Preparation of 1d<smiles>COc1ccc(C(=O)c2ccccc2)cc1Cl</smiles>

(3-Chloro-4-methoxyphenyl)(phenyl)methanone (1d). To a solution of benzoylchloride (1.15 g, $8.04 \mathrm{mmol})$ and $\mathrm{AlMe}_{3}(1.30 \mathrm{~g}, 9.78 \mathrm{mmol})$ in $\mathrm{CH}_{2} \mathrm{Cl}_{2}(10 \mathrm{~mL})$ was slowly added 2-chloroanisole (S1, $1.16 \mathrm{~g}, 8.11 \mathrm{mmol})$ at $0{ }^{\circ} \mathrm{C}$. After stirring at the temperature for $3 \mathrm{~h}$, the reaction mixture was quenched with $\mathrm{H}_{2} \mathrm{O}$. The organic layer was washed with $\mathrm{H}_{2} \mathrm{O}$ and brine, dried over $\mathrm{MgSO}_{4}$, and concentrated. The residue was purified by coliumn chromatography on silica gel (hexane:AcOEt $=10: 1$ ) to give 1d (1.63 g, $6.59 \mathrm{mmol}, 82 \%$ ) as a white solid: $\mathrm{Mp} \mathrm{80-81}{ }^{\circ} \mathrm{C}$ (hexane) (lit. $\left.77-79^{\circ} \mathrm{C}^{8}\right) .{ }^{1} \mathrm{H} \mathrm{NMR}(400 \mathrm{MHz}$, $\left.\mathrm{CDCl}_{3} / \mathrm{TMS}\right) \delta 7.90(\mathrm{~d}, 1 \mathrm{H}, J=2.4 \mathrm{~Hz}), 7.78-7.74(\mathrm{~m}, 3 \mathrm{H}), 7.59(\mathrm{t}, 1 \mathrm{H}, J=7.3 \mathrm{~Hz}), 7.49(\mathrm{t}, 2 \mathrm{H}, J=$ $7.6 \mathrm{~Hz}), 7.00(\mathrm{~d}, 1 \mathrm{H}, J=8.8 \mathrm{~Hz}), 4.00(\mathrm{~s}, 3 \mathrm{H}) .{ }^{13} \mathrm{C} \mathrm{NMR}\left(100 \mathrm{MHz}, \mathrm{CDCl}_{3}\right) \delta 56.3,111.1,122.6$, 128.3, 129.6, 130.69, 130.70, 132.2, 132.3, 137.6, 158.4, 194.3. LRMS (EI) $m / z: 246\left(\mathrm{M}^{+}\right)$. HRMS Calcd. for $\mathrm{C}_{14} \mathrm{H}_{11} \mathrm{ClO}_{2}$ : 246.0448, found: 246.0443. IR (neat): 3010, 2843, 1645, 1592, 1268, 1157, $1060,966,794,734 \mathrm{~cm}^{-1}$. The spectra data matched those reported in the literature. ${ }^{8}$ 
Scheme S3. Preparation of 19<smiles>COc1ccc(C(=O)C(C(F)(F)F)C(F)(F)F)cc1</smiles>

S2

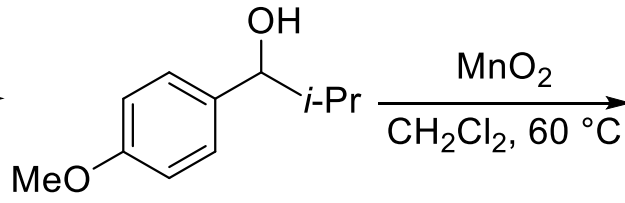

S3<smiles>CCCC(=O)c1ccc(OC)cc1</smiles>

$1 \mathrm{~g}$

1-(4-Methoxyphenyl)-2-methylpropan-1-one (1g). To a solution of $\mathbf{S 2}$ (994 $\mathrm{mg}, 7.30 \mathrm{mmol})$ in THF $(8 \mathrm{~mL})$ was added dropwise $i$-PrMgCl in THF $(2.0 \mathrm{M}, 4.4 \mathrm{~mL}, 8.8 \mathrm{mmol})$ at $0{ }^{\circ} \mathrm{C}$. After stirring at room temperature for $3 \mathrm{~h}$, the reaction mixture was quenched with saturated aqueous $\mathrm{NH}_{4} \mathrm{Cl}$ and extracted with AcOEt. The combined organic layer was washed with brine, dried over $\mathrm{Na}_{2} \mathrm{SO}_{4}$, and concentrated. The residue was purified by column chromatography on silica gel (hexane:AcOEt $=$ 10:1) to afford alcohol $\mathbf{S 3}(1.18 \mathrm{~g}, 6.55 \mathrm{mmol}, 90 \%)$.

A solution of $\mathbf{S 3}(1.18 \mathrm{~g}, 6.55 \mathrm{mmol})$ and $\mathrm{MnO}_{2}(4.06 \mathrm{~g}, 46.7 \mathrm{mmol})$ in $\mathrm{CH}_{2} \mathrm{Cl}_{2}(15 \mathrm{~mL})$ was stirred at $60{ }^{\circ} \mathrm{C}$ for $11 \mathrm{~h}$. The resulting mixture was filtered $\left(\right.$ Celite $\left.{ }^{\circledR}, \mathrm{CH}_{2} \mathrm{Cl}_{2}\right)$ and concentrated. The residue was purified by silica gel column chromatography (hexane:AcOEt = 20:1) to give 1g $(697 \mathrm{mg}, 3.91 \mathrm{mmol}$, $60 \%$ ) as a colorless oil. ${ }^{1} \mathrm{H}$ NMR (400 MHz, $\left.\mathrm{CDCl}_{3} / \mathrm{TMS}\right) \delta 7.95(\mathrm{~d}, 2 \mathrm{H}, J=9.3 \mathrm{~Hz}), 6.94(\mathrm{~d}, 2 \mathrm{H}, J=$ $9.3 \mathrm{~Hz}), 3.87$ (s, 3H), 3.52 (sept, $1 \mathrm{H}, J=7.0 \mathrm{~Hz}), 1.21$ (d, 6H, $J=6.8 \mathrm{~Hz}) .{ }^{13} \mathrm{C}$ NMR $(100 \mathrm{MHz}$, $\left.\mathrm{CDCl}_{3}\right) \delta 19.2,34.8,55.3,113.7,129.0,130.5,163.2,203.0$. LRMS (EI) $\mathrm{m} / \mathrm{z}: 178\left(\mathrm{M}^{+}\right)$. HRMS Calcd. for $\mathrm{C}_{11} \mathrm{H}_{14} \mathrm{O}_{2}$ : 178.0994, found: 178.1013. IR (neat): 2970, 2935, 1672, 1597, 1510, 1226, 1157, 1030, $979,841 \mathrm{~cm}^{-1}$. The spectra data matched those reported in the literature. ${ }^{9}$

Scheme S4. Preparation of $1 \mathrm{~m}$<smiles>Clc1nccc2ccccc12</smiles>

S4

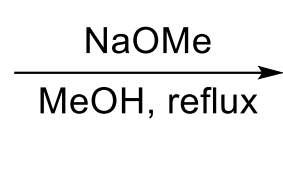<smiles>COc1nccc2ccccc12</smiles>

$1 \mathrm{~m}$

1-Methoxyisoquinoline (1 $\mathbf{m})$. To a solution of $\mathrm{NaOMe}(981 \mathrm{mg}, 18.2 \mathrm{mmol})$ in $\mathrm{MeOH}(6 \mathrm{~mL})$ was added 1-chloroisoquinoline (S4, $499 \mathrm{mg}, 3.05 \mathrm{mmol})$. The mixture was refluxed for $22.5 \mathrm{~h}$. The solvent was removed in vacuo, and $\mathrm{H}_{2} \mathrm{O}$ was added to the residue. The organic materials were extracted with AcOEt. The combined organic layer was washed with brine, dried over $\mathrm{MgSO}_{4}$, and concentrated. The residue was purified by column chromatography on silica gel (hexane:AcOEt $=10: 1)$ to give $\mathbf{1 m}(455$ mg, $2.86 \mathrm{mmol}, 94 \%$ ) as a colorless oil. ${ }^{1} \mathrm{H}$ NMR (400 MHz, $\left.\mathrm{CDCl}_{3} / \mathrm{TMS}\right) \delta 8.25$ (d, $1 \mathrm{H}, J=8.3 \mathrm{~Hz}$ ), $8.00(\mathrm{~d}, 1 \mathrm{H}, J=5.8 \mathrm{~Hz}), 7.74(\mathrm{~d}, 1 \mathrm{H}, J=7.8 \mathrm{~Hz}), 7.66(\mathrm{dt}, 1 \mathrm{H}, J=1.3,7.6 \mathrm{~Hz}), 7.54(\mathrm{dt}, 1 \mathrm{H}, J=1.2$, $7.7 \mathrm{~Hz}), 7.22(\mathrm{~d}, 1 \mathrm{H}, J=5.9 \mathrm{~Hz}), 4.14(\mathrm{~s}, 3 \mathrm{H}) .{ }^{13} \mathrm{C} \mathrm{NMR}\left(100 \mathrm{MHz}, \mathrm{CDCl}_{3}\right) \delta 53.6,114.9,119.8$, 124.1, 126.1, 126.6, 130.4, 137.8, 139.6, 160.9. LRMS (EI) $m / z: 159\left(\mathrm{M}^{+}\right)$. HRMS Calcd. for $\mathrm{C}_{10} \mathrm{H}_{9} \mathrm{NO}$ : 159.0684 , found: 159.0670. IR (neat): 3386, 2945, 1628, 1570, 1501, 1461, 1365, 1249, $1094,746 \mathrm{~cm}^{-1}$. 
Scheme S5. Preparation of 4 a<smiles>COc1ccccc1-c1ccccc1C=O</smiles>

S5<smiles>COc1ccccc1-c1ccccc1/C=[P+]\c1ccccc1</smiles>

S6<smiles>COc1ccccc1-c1ccccc1CNc1ccccc1</smiles>

4a

$N$-((2'-Methoxy-[1,1'-biphenyl]-2-yl)methyl)aniline (4a). To a solution of S5 (110 mg, $0.519 \mathrm{mmol})$ and HOAc (41.5 mg, $0.691 \mathrm{mmol})$ in $\mathrm{CH}_{2} \mathrm{Cl}_{2}(7 \mathrm{~mL})$ was added a solution of aniline $(49.5 \mathrm{mg}, 0.532$ mmol) in $\mathrm{CH}_{2} \mathrm{Cl}_{2}(3 \mathrm{~mL})$. After stirring at room temperature for $11 \mathrm{~h}$, the mixture was concentrated. The residue was dissolved in THF-MeOH (2:1, $15 \mathrm{~mL})$, to which $\mathrm{NaBH}_{4}(593 \mathrm{mg}, 15.7 \mathrm{mmol})$ was slowly added at $0{ }^{\circ} \mathrm{C}$. After stirring at room temperature for $5 \mathrm{~h}$, the reaction was quenched $\mathrm{NH}_{4} \mathrm{Cl}$ aqueous solution, and the organic materials were extracted with AcOEt. The combined organic layer was washed with brine, dried over $\mathrm{Na}_{2} \mathrm{SO}_{4}$ and concentrated. To the residue was added $1 \mathrm{M}$ aqueous $\mathrm{HCl}(10 \mathrm{~mL})$ and the organic materials were extracted with AcOEt. The combined organic layer was washed with brine, dried over $\mathrm{Na}_{2} \mathrm{SO}_{4}$ and concentrated. The residue was purified by $\mathrm{GPC}\left(\mathrm{CHCl}_{3}\right)$ to give 4a (86.2 mg, $0.298 \mathrm{mmol}, 57 \%)$ as a white solid: $\mathrm{Mp} 135{ }^{\circ} \mathrm{C}\left(\mathrm{CH}_{2} \mathrm{Cl}_{2} /\right.$ hexane). ${ }^{1} \mathrm{H}$ NMR (400 $\left.\mathrm{MHz}, \mathrm{CDCl}_{3} / \mathrm{TMS}\right) \delta 7.51-7.48(\mathrm{~m}, 1 \mathrm{H}), 7.37-7.31(\mathrm{~m}, 3 \mathrm{H}), 7.23-7.17(\mathrm{~m}, 2 \mathrm{H}), 7.11(\mathrm{dt}, 1 \mathrm{H}, J=1.0$, $8.1 \mathrm{~Hz}), 7.02(\mathrm{dt}, 1 \mathrm{H}, J=1.0,7.6 \mathrm{~Hz}), 6.95(\mathrm{~d}, 1 \mathrm{H}, J=8.3 \mathrm{~Hz}), 6.65(\mathrm{t}, 1 \mathrm{H}, J=7,6 \mathrm{~Hz}), 6.51(\mathrm{~d}, 2 \mathrm{H}$, $J=7.8 \mathrm{~Hz}), 4.16(\mathrm{~d}, 1 \mathrm{H}, J=15.1 \mathrm{~Hz}), 4.08(\mathrm{~d}, 1 \mathrm{H}, J=14.6 \mathrm{~Hz}), 4.01$ (br s, $1 \mathrm{H}), 3.78(\mathrm{~s}, 3 \mathrm{H}) .{ }^{13} \mathrm{C}$ NMR (100 MHz, $\left.\mathrm{CDCl}_{3}\right) \delta 46.1,55.4,110.6,112.7,117.1,120.7,126.9,127.5,127.7,129.0,129.1$, $129.8,130.3,130.9,137.8,137.9,148.3,156.4$. LRMS (EI) $m / z: 289\left(\mathrm{M}^{+}\right)$. HRMS Calcd. for $\mathrm{C}_{20} \mathrm{H}_{19} \mathrm{NO}: 289.1467$, found: 289.1493. IR (neat): 3425, 2956, 1598, 1507, 1478, 1429, 1333, 1275, $1027,750 \mathrm{~cm}^{-1}$.

Scheme S6. Preparation of $\mathbf{4 b}$<smiles>COc1ccccc1-c1ccccc1C(C)(C)C=O</smiles>

S7

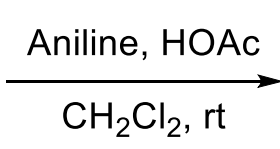<smiles>COc1ccccc1-c1ccccc1C(C)(C)/C=N/c1ccccc1</smiles>

S8

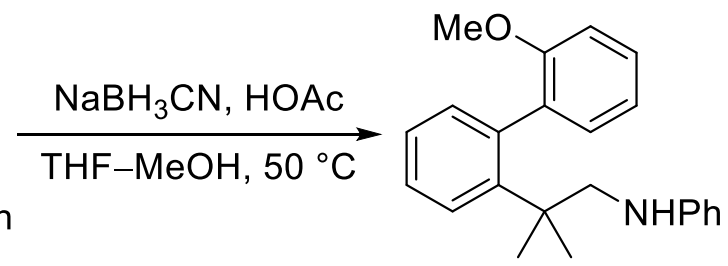

4b

$\mathbf{N}$-(2-(2'-Methoxy-[1,1'-biphenyl]-2-yl)-2-methylpropyl)aniline (4b). To a solution of $\mathbf{S 7}$ (260 mg, $1.02 \mathrm{mmol})$ and $\mathrm{HOAc}(89.1 \mathrm{mg}, 1.48 \mathrm{mmol})$ in $\mathrm{CH}_{2} \mathrm{Cl}_{2}(13 \mathrm{~mL})$ was added a solution of aniline (101 $\mathrm{mg}, 1.09 \mathrm{mmol})$ in $\mathrm{CH}_{2} \mathrm{Cl}_{2}(7 \mathrm{~mL})$. After stirring at room temperature for $10 \mathrm{~h}$, the mixture was concentrated. The residue was dissolved in THF-MeOH (1:1, $6 \mathrm{~mL})$, to which HOAc (101 mg, 1.68 $\mathrm{mmol})$ and $\mathrm{NaBH}_{3} \mathrm{CN}(109 \mathrm{mg}, 1.74 \mathrm{mmol})$ were slowly added in sequence at $0{ }^{\circ} \mathrm{C}$. The resulting mixture was stirred at $50{ }^{\circ} \mathrm{C}$ for $3 \mathrm{~h}$. Then, saturated $\mathrm{Na}_{2} \mathrm{CO}_{3}$ solution $(3 \mathrm{~mL})$ was added, the organic 
phase was separated and the aquous layer was extracted with AcOEt. The collected organic layer washed with brine, dried over $\mathrm{Na}_{2} \mathrm{SO}_{4}$ and concentrated. The residue was purified by column chromatography on silica gel (hexane:AcOEt $=40: 1)$ to give $\mathbf{4 b}(259 \mathrm{mg}, 0.781 \mathrm{mmol}, 76 \%)$ as a white solid: $\mathrm{Mp} 78-79{ }^{\circ} \mathrm{C}\left(\mathrm{CH}_{2} \mathrm{Cl}_{2} /\right.$ hexane). ${ }^{1} \mathrm{H}$ NMR (400 MHz, $\left.\mathrm{CDCl}_{3} / \mathrm{TMS}\right) \delta 7.52(\mathrm{~d}, 1 \mathrm{H}, J=8.3 \mathrm{~Hz})$, 7.37-7.22 (m, 3H), 7.15-7.07 (m, 3H), $7.01(\mathrm{dd}, 1 \mathrm{H}, J=1.4,7.3 \mathrm{~Hz}), 6.91$ (t, 1H, $J=7.6 \mathrm{~Hz}), 6.85$ (d, $1 \mathrm{H}, J=8.3 \mathrm{~Hz}), 6.11(\mathrm{t}, 1 \mathrm{H}, J=7.3 \mathrm{~Hz}), 6.43(\mathrm{~d}, 2 \mathrm{H}, J=7.8 \mathrm{~Hz}), 3.63(\mathrm{~s}, 3 \mathrm{H}), 3.50($ br s, $1 \mathrm{H}), 3.10-$ $3.04(\mathrm{~m}, 1 \mathrm{H}), 3.01-2.96(\mathrm{~m}, 1 \mathrm{H}), 1.31(\mathrm{~s}, 3 \mathrm{H}), 1.25(\mathrm{~s}, 3 \mathrm{H}) .{ }^{13} \mathrm{C} \mathrm{NMR}\left(100 \mathrm{MHz}, \mathrm{CDCl}_{3}\right) \delta 28.8,28.9$, 40.7, 55.0, 55.2, 110.3, 112.5, 116.5, 119.5, 125.8, 127.4, 128.1, 128.5, 128.9, 131.5, 132.9, 133.0, 138.4, 143.7, 148.9, 156.6. LRMS (EI) $m / z: 331\left(\mathrm{M}^{+}\right)$. HRMS Calcd. for $\mathrm{C}_{23} \mathrm{H}_{25} \mathrm{NO}$ : 331.1936, found: 331.1913. IR (neat): 3369, 2964, 2869, 1600, 1503, 1476, 1248, 1119, 1024, $746 \mathrm{~cm}^{-1}$.

General procedure of $t$-Bu-P4 catalyzes amination of methoxy(hetero)arenes (Table 1 and Figures 2 and 3).

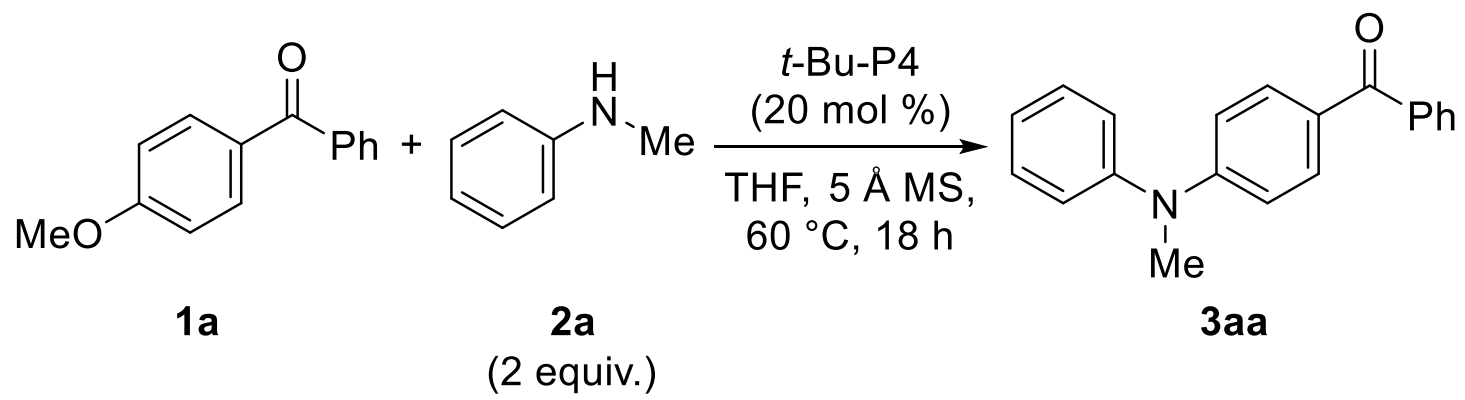

4-(Methyl(phenyl)amino)phenyl)(phenyl)methanone (3aa). In a glove box under an Ar atmosphere, to a mixture of $1 \mathbf{a}(41.4 \mathrm{mg}, 0.195 \mathrm{mmol})$ and $\mathbf{2 a}(42.9 \mathrm{mg}, 0.400 \mathrm{mmol})$ in THF $(0.7 \mathrm{~mL})$ were added $t$-Bu-P4 solution $(50 \mu \mathrm{L}, 0.8 \mathrm{M}$ in hexane, $0.04 \mathrm{mmol})$ and $5 \AA$ molecular sieves $(100 \mathrm{mg})$ in an ovendried vial equipped with a stirrer bar. The vial was sealed with a cap containing an inner Teflon film, and taken outside the glove box. After $60{ }^{\circ} \mathrm{C}$ for $18 \mathrm{~h}$, saturated $\mathrm{NH}_{4} \mathrm{Cl}$ aqueous solution $(1 \mathrm{~mL})$ was added to the reaction mixture at $0{ }^{\circ} \mathrm{C}$. The mixture was extracted with AcOEt $(10 \mathrm{~mL} \times 3)$. The combined organic layer was washed with brine $(10 \mathrm{~mL})$, dried over $\mathrm{Na}_{2} \mathrm{SO}_{4}$, and concentrated. The crude material was purified by column chromatography on silica gel (toluene) to afford 3aa (54.6 mg, $0.190 \mathrm{mmol}, 97 \%$ ) as a yellow solid: $\mathrm{Mp} 79{ }^{\circ} \mathrm{C}$ (hexane) (lit. 81-81 ${ }^{\circ} \mathrm{C}^{10 \mathrm{c}}$ ). ${ }^{1} \mathrm{H}$ NMR (400 MHz, $\left.\mathrm{CDCl}_{3} / \mathrm{TMS}\right) \delta 7.75-7.72(\mathrm{~m}, 4 \mathrm{H}), 7.53(\mathrm{t}, 1 \mathrm{H}, J=7.3 \mathrm{~Hz}), 7.47-7.40(\mathrm{~m}, 4 \mathrm{H}), 7.25-7.21(\mathrm{~m}, 3 \mathrm{H}), 6.79$ $(\mathrm{d}, 2 \mathrm{H}, J=9.2 \mathrm{~Hz}), 3.40(\mathrm{~s}, 3 \mathrm{H}) .{ }^{13} \mathrm{C} \mathrm{NMR}\left(100 \mathrm{MHz}, \mathrm{CDCl}_{3}\right) \delta 40.2,113.3,125.6,126.1,126.6$, 128.0, 129.5, 129.8, 131.3, 132.3, 138.9, 147.2, 152.5, 195.1. LRMS (EI) $m / z: 287\left(\mathrm{M}^{+}\right)$. HRMS Calcd. for $\mathrm{C}_{20} \mathrm{H}_{17} \mathrm{NO}$ : 287.1310, found: 287.1305. IR (neat): 3052, 2909, 1628, 1587, 1575, 1486, 1261, 1112, $835,753 \mathrm{~cm}^{-1}$. The spectra data matched those reported in the literature. ${ }^{10}$ 
<smiles>Cc1ccc(N(C)c2ccc(C(=O)c3ccccc3)cc2)cc1</smiles>

(4-(Methyl(p-tolyl)amino)phenyl)(phenyl)methanone (3ab). According to the general procedure analogous to that described for 3aa, 3ab $(53.2 \mathrm{mg}, 0.177 \mathrm{mmol}, 90 \%)$ was obtained from $1 \mathbf{a}(41.8 \mathrm{mg}$, $0.197 \mathrm{mmol})$ as a yellow oil. ${ }^{1} \mathrm{H}$ NMR (400 MHz, $\left.\mathrm{CDCl}_{3} / \mathrm{TMS}\right) \delta 7.74-7.71(\mathrm{~m}, 4 \mathrm{H}), 7.52(\mathrm{t}, 1 \mathrm{H}, J=$ $7.3 \mathrm{~Hz}), 7.44$ (t, 2H, $J=7.3 \mathrm{~Hz}), 7.23(\mathrm{~d}, 2 \mathrm{H}, J=8.3 \mathrm{~Hz}), 7.13(\mathrm{~d}, 2 \mathrm{H}, J=8.3 \mathrm{~Hz}), 6.73(\mathrm{~d}, 2 \mathrm{H}, J=8.8$ $\mathrm{Hz}), 3.36(\mathrm{~s}, 3 \mathrm{H}), 2.38(\mathrm{~s}, 3 \mathrm{H}) .{ }^{13} \mathrm{C} \mathrm{NMR}\left(100 \mathrm{MHz}, \mathrm{CDCl}_{3}\right) \delta 20.9,40.2,112.7,126.2,126.3,127.9$, 129.4, 130.5, 131.2, 132.3, 135.7, 139.0, 144.6, 152.7, 195.0. LRMS (EI) m/z: $301\left(\mathrm{M}^{+}\right)$. HRMS Calcd. for $\mathrm{C}_{21} \mathrm{H}_{19} \mathrm{NO}: 301.1467$, found: 301.1462. IR (neat): 3025, 2920, 1642, 1587, 1576, 1507, 1316, 1279, $1192,824 \mathrm{~cm}^{-1}$.<smiles>COc1ccc(N(C)c2ccc(C(=O)c3ccccc3)cc2)cc1</smiles>

(4-((4-Methoxyphenyl)(methyl)amino)phenyl)(phenyl)methanone (3ac). According to the general procedure analogous to that described for 3aa, except that the crude material was purified by GPC $\left(\mathrm{CHCl}_{3}\right), 3 \mathbf{a c}(48.5 \mathrm{mg}, 0.153 \mathrm{mmol}, 76 \%)$ was obtained from $1 \mathbf{a}(42.7 \mathrm{mg}, 0.201 \mathrm{mmol})$ as a yellow solid: Mp $82{ }^{\circ} \mathrm{C}\left(\mathrm{CH}_{2} \mathrm{Cl}_{2} /\right.$ hexane). ${ }^{1} \mathrm{H}$ NMR (400 MHz, $\left.\mathrm{CDCl}_{3} / \mathrm{TMS}\right) \delta$ 7.74-7.70 (m, 4H), 7.54-7.49 (m, 1H), 7.44 (d, 2H, $J=7.3 \mathrm{~Hz}), 7.16(\mathrm{~d}, 2 \mathrm{H}, J=8.8 \mathrm{~Hz}), 6.96(\mathrm{~d}, 2 \mathrm{H}, J=9.2 \mathrm{~Hz}), 6.67(\mathrm{~d}, 2 \mathrm{H}, J=$ $8.8 \mathrm{~Hz}), 3.84$ (s, 3H), 3.34 (s, 3H). ${ }^{13} \mathrm{C} \mathrm{NMR}\left(100 \mathrm{MHz}, \mathrm{CDCl}_{3}\right) \delta 40.3,55.4,112.2,115.1,125.8$, 128.0, 128.1, 129.4, 131.1, 132.4, 139.1, 140.0, 153.0, 157.8, 195.0. LRMS (EI) $\mathrm{m} / z: 317\left(\mathrm{M}^{+}\right)$. HRMS Calcd. for $\mathrm{C}_{21} \mathrm{H}_{19} \mathrm{NO}_{2}: 317.1416$, found: 317.1436. IR (neat): 2999, 2959, 2835, 1629, 1594, 1506, $1242,1032,831,702 \mathrm{~cm}^{-1}$.<smiles>CN(c1ccc(Cl)cc1)c1ccc(C(=O)c2ccccc2)cc1</smiles>

(4-((4-Chlorophenyl)(methyl)amino)phenyl)(phenyl)methanone (3ad). According to the general procedure analogous to that described for 3aa, 3ad $(60.3 \mathrm{mg}, 0.187 \mathrm{mmol}, 96 \%)$ was obtained from 1a (41.6 mg, $0.196 \mathrm{mmol})$ as a yellow solid: $\mathrm{Mp} 87-88{ }^{\circ} \mathrm{C}\left(\mathrm{CH}_{2} \mathrm{Cl}_{2} /\right.$ hexane). ${ }^{1} \mathrm{H} \mathrm{NMR}(400 \mathrm{MHz}$, $\left.\mathrm{CDCl}_{3} / \mathrm{TMS}\right) \delta 7.78-7.72(\mathrm{~m}, 4 \mathrm{H}), 7.54(\mathrm{t}, 1 \mathrm{H}, J=7.3 \mathrm{~Hz}), 7.45(\mathrm{t}, 2 \mathrm{H}, J=7.3 \mathrm{~Hz}), 7.37(\mathrm{~d}, 2 \mathrm{H}, J=$ $8.8 \mathrm{~Hz}), 7.17(\mathrm{~d}, 2 \mathrm{H}, J=8.8 \mathrm{~Hz}), 6.81(\mathrm{~d}, 2 \mathrm{H}, J=9.3 \mathrm{~Hz}), 3.37(\mathrm{~s}, 3 \mathrm{H}) .{ }^{13} \mathrm{C} \mathrm{NMR}\left(100 \mathrm{MHz}, \mathrm{CDCl}_{3}\right)$ 
$\delta 40.2,114.0,126.9,127.4,128.0,129.5,129.9,130.5,131.4,132.3,138.7,145.8,152.1,195.1$. LRMS (EI) $m / z: 321\left(\mathrm{M}^{+}\right)$. HRMS Calcd. for $\mathrm{C}_{20} \mathrm{H}_{16} \mathrm{ClNO}$ : 321.0920, found: 321.0918. IR (neat): 2908, 1629, $1598,1490,1286,1260,1104,923,831,740 \mathrm{~cm}^{-1}$.<smiles>CN(c1ccc(Br)cc1)c1ccc(C(=O)c2ccccc2)cc1</smiles>

(4-((4-Bromophenyl)(methyl)amino)phenyl)(phenyl)methanone (3ae). According to the general procedure analogous to that described for 3aa, except that the crude material was purified by GPC $\left(\mathrm{CHCl}_{3}\right)$, 3ae $(61.8 \mathrm{mg}, 0.169 \mathrm{mmol}, 84 \%)$ was obtained from 1a $(42.8 \mathrm{mg}, 0.202 \mathrm{mmol})$ as a beige solid: $\mathrm{Mp} 78{ }^{\circ} \mathrm{C}\left(\mathrm{CH}_{2} \mathrm{Cl}_{2} /\right.$ hexane). ${ }^{1} \mathrm{H} \mathrm{NMR}\left(400 \mathrm{MHz}, \mathrm{CDCl}_{3} / \mathrm{TMS}\right) \delta$ 7.78-7.72 (m, 4H), 7.57-7.49 $(\mathrm{m}, 3 \mathrm{H}), 7.46(\mathrm{t}, 2 \mathrm{H}, J=7.3 \mathrm{~Hz}), 7.11(\mathrm{~d}, 2 \mathrm{H}, J=8.8 \mathrm{~Hz}), 6.83(\mathrm{~d}, 2 \mathrm{H}, J=8.8 \mathrm{~Hz}), 3.37(\mathrm{~s}, 3 \mathrm{H}) .{ }^{13} \mathrm{C}$ NMR $\left(100 \mathrm{MHz}, \mathrm{CDCl}_{3}\right) \delta 40.1,114.2,118.1,127.1,127.5,128.0,129.5,131.4,132.2,132.8,138.7$, 146.3, 152.0, 195.0. LRMS (EI) $m / z$ : $366\left(\mathrm{M}^{+}\right)$. HRMS Calcd. for $\mathrm{C}_{20} \mathrm{H}_{16} \mathrm{BrNO}$ : 365.0415 , found: 365.0431. IR (neat): 2911, 2832, 1643, 1599, 1484, 1318, 1285, 1138, 1001, $824 \mathrm{~cm}^{-1}$.<smiles>CCN(c1ccccc1)c1ccc(C(=O)c2ccccc2)cc1</smiles>

(4-(Ethyl(phenyl)amino)phenyl)(phenyl)methanone (3af). According to the general procedure analogous to that described for 3aa, except that the reaction was conducted at $90{ }^{\circ} \mathrm{C}, \mathbf{3 a f}(35.9 \mathrm{mg}$, $0.119 \mathrm{mmol}, 60 \%)$ was obtained from $1 \mathrm{a}(42.0 \mathrm{mg}, 0.198 \mathrm{mmol})$ as a yellow oil. ${ }^{1} \mathrm{H} \mathrm{NMR}(400 \mathrm{MHz}$, $\left.\mathrm{CDCl}_{3} / \mathrm{TMS}\right) \delta 7.76-7.69(\mathrm{~m}, 4 \mathrm{H}), 7.52(\mathrm{t}, 1 \mathrm{H}, J=7.6 \mathrm{~Hz}), 7.47-7.40(\mathrm{~m}, 4 \mathrm{H}), 7.28-7.21(\mathrm{~m}, 3 \mathrm{H})$, $6.72(\mathrm{~d}, 2 \mathrm{H}, J=8.8 \mathrm{~Hz}), 3.83(\mathrm{q}, 2 \mathrm{H}, J=7.2 \mathrm{~Hz}), 1.27(\mathrm{t}, 3 \mathrm{H}, J=7.1 \mathrm{~Hz}) .{ }^{13} \mathrm{C} \mathrm{NMR}\left(100 \mathrm{MHz}, \mathrm{CDCl}_{3}\right)$ $\delta 12.5,46.7,113.1,126.0,126.2,127.2$, 128.0, 129.4, 129.9, 131.2, 132.5, 139.0, 145.7, 151.7, 195.0. LRMS (EI) $m / z$ : $301\left(\mathrm{M}^{+}\right)$. HRMS Calcd. for $\mathrm{C}_{21} \mathrm{H}_{19} \mathrm{NO}$ : 301.1467, found: 301.1461. IR (neat): 3062, $2974,1641,1601,1584,1492,1316,1269,1151,921 \mathrm{~cm}^{-1}$. The spectra data matched those reported in the literature. ${ }^{11}$<smiles>O=C(c1ccccc1)c1ccc(N2CCc3ccccc32)cc1</smiles> 
(4-(Indolin-1-yl)phenyl)(phenyl)methanone (3ag). According to the general procedure analogous to that described for 3aa, except that the crude material was purified by column chromatography on silica gel (hexane:AcOEt = 10:1), $\mathbf{3 a g}(51.8 \mathrm{mg}, 0.173 \mathrm{mmol}, 87 \%)$ was obtained from 1a (42.1 $\mathrm{mg}, 0.198$ mmol) as a yellow solid: Mp $102{ }^{\circ} \mathrm{C}\left(\mathrm{CH}_{2} \mathrm{Cl}_{2} /\right.$ hexane). ${ }^{1} \mathrm{H}$ NMR (400 MHz, $\left.\mathrm{CDCl}_{3} / \mathrm{TMS}\right) \delta 7.87(\mathrm{~d}$, $2 \mathrm{H}, J=8.8 \mathrm{~Hz}), 7.80-7.76(\mathrm{~m}, 2 \mathrm{H}), 7.59-7.53(\mathrm{~m}, 1 \mathrm{H}), 7.48(\mathrm{t}, 2 \mathrm{H}, J=7.6 \mathrm{~Hz}), 7.34(\mathrm{~d}, 1 \mathrm{H}, J=8.3$ $\mathrm{Hz}), 7.28-7.21(\mathrm{~m}, 3 \mathrm{H}), 7.15(\mathrm{t}, 1 \mathrm{H}, J=8.1 \mathrm{~Hz}), 6.86(\mathrm{t}, 1 \mathrm{H}, J=7.6 \mathrm{~Hz}), 4.06(\mathrm{t}, 2 \mathrm{H}, J=8.3 \mathrm{~Hz}), 3.19$ $(\mathrm{t}, 2 \mathrm{H}, J=8.5 \mathrm{~Hz}) .{ }^{13} \mathrm{C} \mathrm{NMR}\left(100 \mathrm{MHz}, \mathrm{CDCl}_{3}\right) \delta 28.0,51.8,109.8,115.1,120.5,125.3,127.1,128.1$, 128.5, 129.6, 131.5, 132.0, 132.2, 138.6, 145.1, 147.7, 195.1. LRMS (EI) m/z: $299\left(\mathrm{M}^{+}\right)$. HRMS Calcd. for $\mathrm{C}_{21} \mathrm{H}_{17} \mathrm{NO}$ : 299.1310, found: 299.1303. IR (neat): 3409, 3035, 2923, 1641, 1587, 1481, 1270, 1146, $833,745 \mathrm{~cm}^{-1}$. The spectra data matched those reported in the literature. ${ }^{12}$<smiles>Cc1ccc2c(c1)CCN2c1ccc(C(=O)c2ccccc2)cc1</smiles>

(4-(5-Methylindolin-1-yl)phenyl)(phenyl)methanone (3ah). According to the general procedure analogous to that described for 3aa, 3ah $(58.0 \mathrm{mg}, 0.185 \mathrm{mmol}, 94 \%)$ was obtained from 1a (41.8 $\mathrm{mg}$, $0.197 \mathrm{mmol})$ as a orange solid: $\mathrm{Mp} 145^{\circ} \mathrm{C}\left(\mathrm{CH}_{2} \mathrm{Cl}_{2} /\right.$ hexane $) .{ }^{1} \mathrm{H}$ NMR (400 MHz, $\left.\mathrm{CDCl}_{3} / \mathrm{TMS}\right) \delta 7.86$ $(\mathrm{d}, 2 \mathrm{H}, J=8.8 \mathrm{~Hz}), 7.77$ (d, 2H, $J=7.3 \mathrm{~Hz}), 7.58-7.51(\mathrm{~m}, 1 \mathrm{H}), 7.47$ (t, 2H, $J=7.3 \mathrm{~Hz}), 7.26-7.19$ (m, $3 \mathrm{H}), 7.05(\mathrm{~s}, 1 \mathrm{H}), 6.95(\mathrm{~d}, 1 \mathrm{H}, J=8.3 \mathrm{~Hz}), 4.03(\mathrm{t}, 2 \mathrm{H}, J=8.5 \mathrm{~Hz}), 3.15(\mathrm{t}, 2 \mathrm{H}, J=8.5 \mathrm{~Hz}), 2.30$ (s, $3 \mathrm{H}) .{ }^{13} \mathrm{C} \mathrm{NMR}\left(100 \mathrm{MHz}, \mathrm{CDCl}_{3}\right) \delta 20.7,27.9,51.8,109.7,114.6,126.1,127.3,128.0,128.1,129.5$, 130.1, 131.5, 132.30, 132.32, 138.7, 142.6, 147.8, 195.0. LRMS (EI) m/z: $313\left(\mathrm{M}^{+}\right)$. HRMS Calcd. for $\mathrm{C}_{22} \mathrm{H}_{19} \mathrm{NO}: 313.1467$, found: 313.1489. IR (neat): 3022, 2916, 2854, 1643, 1585, 1514, 1487, 1276, $1149,821 \mathrm{~cm}^{-1}$. The spectra data matched those reported in the literature. ${ }^{12}$<smiles>O=C(c1ccccc1)c1ccc(N2CCc3cc(Br)ccc32)cc1</smiles>

(4-(5-Bromoindolin-1-yl)phenyl)(phenyl)methanone (3ai). According to the general procedure analogous to that described for 3aa, except that the crude material was purified by $\mathrm{GPC}\left(\mathrm{CHCl}_{3}\right)$, 3ai (66.1 mg, $0.175 \mathrm{mmol}, 87 \%)$ was obtained from $1 \mathrm{a}(42.5 \mathrm{mg}, 0.200 \mathrm{mmol})$ as a yellow solid: Mp 145 $147{ }^{\circ} \mathrm{C}$ (hexane). ${ }^{1} \mathrm{H}$ NMR (400 MHz, $\left.\mathrm{CDCl}_{3} / \mathrm{TMS}\right) \delta 7.87(\mathrm{~d}, 2 \mathrm{H}, J=8.8 \mathrm{~Hz}), 7.78(\mathrm{~d}, 2 \mathrm{H}, J=8.0$ $\mathrm{Hz}), 7.60-7.55(\mathrm{~m}, 1 \mathrm{H}), 7.48(\mathrm{t}, 2 \mathrm{H}, J=7.3 \mathrm{~Hz}), 7.31(\mathrm{~s}, 1 \mathrm{H}), 7.24-7.16(\mathrm{~m}, 4 \mathrm{H}), 4.07$ (t, $2 \mathrm{H}, J=8.3$ $\mathrm{Hz}), 3.17(\mathrm{t}, 2 \mathrm{H}, J=8.5 \mathrm{~Hz}) .{ }^{13} \mathrm{C} \mathrm{NMR}\left(100 \mathrm{MHz}, \mathrm{CDCl}_{3}\right) \delta 27.7,51.9,110.8,112.0,115.2,128.1$, 128.2, 129.0, 129.6, 129.8, 131.7, 132.2, 134.4, 138.4, 144.3, 147.1, 195.0. LRMS (EI) $m / z: 378\left(\mathrm{M}^{+}\right)$. 
HRMS Calcd. for $\mathrm{C}_{21} \mathrm{H}_{16} \mathrm{BrNO}$ : 377.0415, found: 377.0402. IR (neat): 3054, 2867, 1643, 1584, 1516, $1481,1282,1153,789,736 \mathrm{~cm}^{-1}$.<smiles>CC1Cc2ccccc2N1c1ccc(C(=O)c2ccccc2)cc1</smiles>

(4-(2-Methylindolin-1-yl)phenyl)(phenyl)methanone (3aj). According to the general procedure analogous to that described for 3aa, except that the crude material was purified by $\mathrm{GPC}\left(\mathrm{CHCl}_{3}\right), \mathbf{3 a j}$ (58.2 mg, $0.186 \mathrm{mmol}, 95 \%)$ was obtained from $1 \mathrm{a}(41.6 \mathrm{mg}, 0.196 \mathrm{mmol})$ as a yellow oil. ${ }^{1} \mathrm{H} \mathrm{NMR}$ (400 MHz, $\left.\mathrm{CDCl}_{3} / \mathrm{TMS}\right) \delta 7.86(\mathrm{~d}, 2 \mathrm{H}, J=8.8 \mathrm{~Hz}), 7.78(\mathrm{~d}, 2 \mathrm{H}, J=7.5 \mathrm{~Hz}), 7.56(\mathrm{t}, 1 \mathrm{H}, J=7.3 \mathrm{~Hz})$, $7.48(\mathrm{t}, 2 \mathrm{H}, J=7.3 \mathrm{~Hz}), 7.30(\mathrm{~d}, 2 \mathrm{H}, J=8.8 \mathrm{~Hz}), 7.25-7.20(\mathrm{~m}, 2 \mathrm{H}), 7.14(\mathrm{t}, 1 \mathrm{H}, J=7.6 \mathrm{~Hz}), 6.87$ (t, $1 \mathrm{H}, J=7.6 \mathrm{~Hz}), 4.56-4.47(\mathrm{~m}, 1 \mathrm{H}), 3.43(\mathrm{dd}, 1 \mathrm{H}, J=8.6,15.4 \mathrm{~Hz}), 2.71(\mathrm{dd}, 1 \mathrm{H}, J=3.9,15.1 \mathrm{~Hz})$, $1.39(\mathrm{~d}, 3 \mathrm{H}, J=6.4 \mathrm{~Hz}) .{ }^{13} \mathrm{C} \mathrm{NMR}\left(100 \mathrm{MHz}, \mathrm{CDCl}_{3}\right) \delta 20.0,36.8,59.1,110.9,115.9,120.7,125.5$, 127.1, 128.1, 128.9, 129.6, 130.8, 131.5, 132.2, 138.6, 144.6, 147.3, 195.0. LRMS (EI) m/z: $313\left(\mathrm{M}^{+}\right)$. HRMS Calcd. for $\mathrm{C}_{22} \mathrm{H}_{19} \mathrm{NO}: 313.1467$, found: 313.1437. IR (neat): 3047, 2966, 1645, 1586, 1512, $1479,1374,1275,1149,746 \mathrm{~cm}^{-1}$.<smiles>CN(c1ccc(C(=O)c2ccccc2)cc1)c1ccccn1</smiles>

(4-(Methyl(pyridin-2-yl)amino)phenyl)(phenyl)methanone (3ak). According to the general procedure analogous to that described for 3aa, except that the reaction was conducted with $t$-Bu-P4 solution $(75 \mu \mathrm{L}, 0.8 \mathrm{M}$ in hexane, $0.06 \mathrm{mmol})$ and crude material was purified by column chromatography on silica gel (hexane:AcOEt = 3:1), 3ak (30.0 mg, $0.104 \mathrm{mmol}, 52 \%)$ was obtained from $1 \mathrm{a}(42.1 \mathrm{mg}, 0.198 \mathrm{mmol})$ as a yellow solid: $\mathrm{Mp} 80{ }^{\circ} \mathrm{C}\left(\mathrm{CH}_{2} \mathrm{Cl}_{2} /\right.$ hexane). ${ }^{1} \mathrm{H} \mathrm{NMR}(400 \mathrm{MHz}$, $\left.\mathrm{CDCl}_{3} / \mathrm{TMS}\right) \delta 8.33(\mathrm{~d}, 1 \mathrm{H}, J=3.4 \mathrm{~Hz}), 7.85-7.79(\mathrm{~m}, 4 \mathrm{H}), 7.58(\mathrm{t}, 1 \mathrm{H}, J=7.3 \mathrm{~Hz}), 7.52-7.45(\mathrm{~m}$, $3 \mathrm{H}), 7.30(\mathrm{~d}, 2 \mathrm{H}, J=8.8 \mathrm{~Hz}), 6.96(\mathrm{~d}, 1 \mathrm{H}, J=8.8 \mathrm{~Hz}), 6.81(\mathrm{t}, 1 \mathrm{H}, J=6.1 \mathrm{~Hz}), 3.57(\mathrm{~s}, 3 \mathrm{H}) .{ }^{13} \mathrm{C}$ NMR $\left(150 \mathrm{MHz}, \mathrm{THF}-d_{8}\right) \delta 37.0,111.3,115.3,122.0,128.0,129.5,131.3,131.6,132.2,136.6,138.4,148.1$, 150.8, 158.0, 193.6. LRMS (EI) $m / z: 288\left(\mathrm{M}^{+}\right)$. HRMS Calcd. for $\mathrm{C}_{19} \mathrm{H}_{16} \mathrm{~N}_{2} \mathrm{O}: 288.1263$, found: 288.1275. IR (neat): 3055, 2916, 1647, 1598, 1583, 1471, 1276, 1114, 922, $740 \mathrm{~cm}^{-1}$. 
<smiles>CN(c1ccccc1)c1ccc(C(=O)c2ccccc2)cc1</smiles>

(4-(Methyl(pyridin-3-yl)amino)phenyl)(phenyl)methanone (3al). According to the general procedure analogous to that described for 3aa, except that the crude material was purified by column chromatography on silica gel (hexane:AcOEt = 1:2), 3al (50.0 mg, $0.173 \mathrm{mmol}, 87 \%)$ was obtained from 1a(42.3 mg, $0.199 \mathrm{mmol}$ ) as a yellow solid: Mp $72{ }^{\circ} \mathrm{C}$ (hexane). ${ }^{1} \mathrm{H}$ NMR (400 MHz, $\left.\mathrm{CDCl}_{3} / \mathrm{TMS}\right) \delta 8.56(\mathrm{~d}, 1 \mathrm{H}, J=2.4 \mathrm{~Hz}), 8.44(\mathrm{~d}, 1 \mathrm{H}, J=3.9 \mathrm{~Hz}), 7.80-7.73(\mathrm{~m}, 4 \mathrm{H}), 7.58-7.52(\mathrm{~m}$, 2H), $7.46(\mathrm{t}, 2 \mathrm{H}, J=7.6 \mathrm{~Hz}), 7.33(\mathrm{dd}, 1 \mathrm{H}, J=4.6,8.0 \mathrm{~Hz}), 6.87(\mathrm{~d}, 2 \mathrm{H}, J=8.8 \mathrm{~Hz}), 3.43(\mathrm{~s}, 3 \mathrm{H}) .{ }^{13} \mathrm{C}$ NMR (100 MHz, $\left.\mathrm{CDCl}_{3}\right) \delta 40.1,114.7,124.1,128.0,128.3,129.5,131.5,132.0,132.2,138.5,143.7$, 145.7, 146.9, 151.7, 195.0. LRMS (EI) $m / z: 288\left(\mathrm{M}^{+}\right)$. HRMS Calcd. for $\mathrm{C}_{19} \mathrm{H}_{16} \mathrm{~N}_{2} \mathrm{O}: 288.1263$, found: 288.1273. IR (neat): 3062, 2922, 2825, 1629, 1597, 1480, 1426, 1265, 1191, $841 \mathrm{~cm}^{-1}$.<smiles>O=C(c1ccccc1)c1ccc(Nc2ccccc2)cc1</smiles>

Phenyl(4-(phenylamino)phenyl)methanone (3am). According to the general procedure analogous to that described for 3aa, 3am $(8.0 \mathrm{mg}, 0.029 \mathrm{mmol}, 15 \%)$ was obtained from $1 \mathrm{a}(41.5 \mathrm{mg}$, 0.196mmol) as a yellow solid: $\mathrm{Mp} 155-156{ }^{\circ} \mathrm{C}$ (hexane/ $\mathrm{CH}_{2} \mathrm{Cl}_{2}$ ) (lit. 154-156 ${ }^{\circ} \mathrm{C}^{13 \mathrm{a}}$ ). ${ }^{1} \mathrm{H}$ NMR (400 $\left.\mathrm{MHz}, \mathrm{CDCl}_{3} / \mathrm{TMS}\right) \delta 7.80-7.74(\mathrm{~m}, 4 \mathrm{H}), 7.58-7.52(\mathrm{~m}, 1 \mathrm{H}), 7.46(\mathrm{t}, 2 \mathrm{H}, J=7.8 \mathrm{~Hz}), 7.35(\mathrm{~d}, 2 \mathrm{H}, J=$ $7.8 \mathrm{~Hz}), 7.26-7.19(\mathrm{~m}, 2 \mathrm{H}), 7.09(\mathrm{t}, 1 \mathrm{H}, J=7.1 \mathrm{~Hz}), 7.02(\mathrm{~d}, 2 \mathrm{H}, J=8.8 \mathrm{~Hz}), 6.07(\mathrm{~s}, 1 \mathrm{H}) .{ }^{13} \mathrm{C} \mathrm{NMR}$ $\left(150 \mathrm{MHz}, \mathrm{CDCl}_{3}\right) \delta .114 .3,120.7,123.4,128.1,128.7,129.5,129.6,131.5,132.7,138.7,140.6,148.1$, 195.1. LRMS (EI) $m / z: 273\left(\mathrm{M}^{+}\right)$. HRMS Calcd. for $\mathrm{C}_{19} \mathrm{H}_{15} \mathrm{NO}: 273.1154$, found: 273.1165 . IR (neat): $3311,1584,1562,1495,1321,1288,1149,926,843,738 \mathrm{~cm}^{-1}$. The spectra data matched those reported in the literature. ${ }^{13}$<smiles>COc1ccc(Nc2ccc(C(=O)c3ccccc3)cc2)cc1</smiles>

(4-((4-Methoxyphenyl)amino)phenyl)(phenyl)methanone (3an). According to the general procedure analogous to that described for 3aa, except that the crude material was purified by column chromatography on silica gel (hexane:AcOEt = 3:1), 3an (7.4 mg, $0.024 \mathrm{mmol}, 12 \%)$ was obtained 
from 1a (42.4 mg, $0.200 \mathrm{mmol})$ as a yellow solid: $\mathrm{Mp} 134-135^{\circ} \mathrm{C}$ (hexane $\left./ \mathrm{CH}_{2} \mathrm{Cl}_{2}\right)\left(\right.$ lit. $\left.128-130{ }^{\circ} \mathrm{C}^{14 \mathrm{a}}\right)$. ${ }^{1} \mathrm{H}$ NMR (400 MHz, $\left.\mathrm{CDCl}_{3} / \mathrm{TMS}\right) \delta 7.76-7.73(\mathrm{~m}, 4 \mathrm{H}), 7.56-7.51(\mathrm{~m}, 1 \mathrm{H}), 7.45(\mathrm{t}, 2 \mathrm{H}, J=7.3 \mathrm{~Hz})$, $7.16(\mathrm{~d}, 2 \mathrm{H}, J=8.8 \mathrm{~Hz}), 6.91(\mathrm{~d}, 2 \mathrm{H}, J=8.3 \mathrm{~Hz}), 6.84(\mathrm{~d}, 2 \mathrm{H}, J=8.3 \mathrm{~Hz}), 5.89$ (s, 1H), $3.82(\mathrm{~s}, 3 \mathrm{H})$. ${ }^{13} \mathrm{C}$ NMR $\left(150 \mathrm{MHz}, \mathrm{CDCl}_{3}\right) \delta .55 .6,113.0,114.8,124.6,127.7,128.0,129.5,131.4,132.8,133.1$, 138.9, 149.9, 156.7, 195.1. LRMS (EI) $m / z: 303\left(\mathrm{M}^{+}\right)$. HRMS Calcd. for $\mathrm{C}_{20} \mathrm{H}_{17} \mathrm{NO}_{2}$ : 303.1259, found: 301.1255. IR (neat): 3318, 2997, 1587, 1506, 1339, 1234, 1150, 1032, 829, $737 \mathrm{~cm}^{-1}$.

The spectra data matched those reported in the literature. ${ }^{14}$<smiles>CN(c1ccccc1)c1ccc(C#N)cc1</smiles>

4-(Methyl(phenyl)amino)benzonitrile (3ba). According to the general procedure analogous to that described for 3aa, except that the reaction was conducted in cyclohexane at room temperature, $\mathbf{3 b a}$ (28.2 $\mathrm{mg}, 0.135 \mathrm{mmol}, 70 \%)$ was obtained from $\mathbf{1 b}(25.7 \mathrm{mg}, 0.193 \mathrm{mmol})$ as a yellow oil. ${ }^{1} \mathrm{H} \mathrm{NMR}$ (400 MHz, $\left.\mathrm{CDCl}_{3} / \mathrm{TMS}\right) \delta 7.45-7.39(\mathrm{~m}, 4 \mathrm{H}), 7.28-7.24(\mathrm{~m}, 1 \mathrm{H}), 7.20(\mathrm{~d}, 2 \mathrm{H}, J=7.3 \mathrm{~Hz}), 6.72(\mathrm{~d}$, $2 \mathrm{H}, J=9.3 \mathrm{~Hz}), 3.35(\mathrm{~s}, 3 \mathrm{H}) .{ }^{13} \mathrm{C} \mathrm{NMR}\left(100 \mathrm{MHz}, \mathrm{CDCl}_{3}\right) \delta 40.1,99.3,113.8,120.3,126.1,126.4$, 130.0, 133.2, 146.8, 151.9. LRMS (EI) $m / z: 208\left(\mathrm{M}^{+}\right)$. HRMS Calcd. for $\mathrm{C}_{14} \mathrm{H}_{12} \mathrm{~N}_{2}$ : 208.1001, found: 208.1002. IR (neat): 3058, 2889, 2213, 1608, 1589, 1511, 1493, 1352, 1176, $822 \mathrm{~cm}^{-1}$. The spectra data matched those reported in the literature. ${ }^{13 \mathrm{~b}, 15}$<smiles>CN(c1ccccc1)c1ccc([N+](=O)[O-])cc1</smiles>

$N$-Methyl-4-nitro- $N$-phenylaniline (3ca). According to the general procedure analogous to that described for 3aa, except that the reaction was conducted in cyclohexane at room temperature and crude material was purified by preparative thin-layer chromatography of silica gel (toluene), 3ca (39.1 $\mathrm{mg}, 0.171 \mathrm{mmol}, 84 \%)$ was obtained from $1 \mathrm{c}(31.3 \mathrm{mg}, 0.204 \mathrm{mmol})$ as a yellow oil. ${ }^{1} \mathrm{H}$ NMR (400 $\left.\mathrm{MHz}, \mathrm{CDCl}_{3} / \mathrm{TMS}\right) \delta 8.07(\mathrm{~d}, 2 \mathrm{H}, J=9.3 \mathrm{~Hz}), 7.46(\mathrm{t}, 2 \mathrm{H}, J=8.1 \mathrm{~Hz}), 7.31(\mathrm{t}, 1 \mathrm{H}, J=7.6 \mathrm{~Hz}), 7.26-$ $7.19(\mathrm{~m}, 2 \mathrm{H}), 6.68(\mathrm{~d}, 2 \mathrm{H}, J=9.3 \mathrm{~Hz}), 3.41(\mathrm{~s}, 3 \mathrm{H}) .{ }^{13} \mathrm{C} \mathrm{NMR}\left(100 \mathrm{MHz}, \mathrm{CDCl}_{3}\right) \delta 40.5,112.4,125.7$, 126.7, 126.8, 130.2, 138.1, 146.4, 153.7. LRMS (EI) $m / z: 228\left(\mathrm{M}^{+}\right)$. HRMS Calcd. for $\mathrm{C}_{13} \mathrm{H}_{12} \mathrm{~N}_{2} \mathrm{O}_{2}$ : 228.0899, found: 228.0887. IR (neat): 3066, 2906, 1603, 1582, 1502, 1485, 1298, 1192, 1106, $829 \mathrm{~cm}^{-}$ 1 . The spectra data matched those reported in the literature. ${ }^{16}$ 
<smiles>CN(c1ccccc1)c1ccc(C(=O)c2ccccc2)cc1Cl</smiles>

(3-Chloro-4-(methyl(phenyl)amino)phenyl)(phenyl)methanone (3da). According to the general procedure analogous to that described for 3aa, except that the reaction was conducted at room temperature, $3 \mathbf{d a}(53.8 \mathrm{mg}, 0.167 \mathrm{mmol}, 86 \%)$ was obtained from $1 \mathrm{n}(48.2 \mathrm{mg}, 0.195 \mathrm{mmol})$ as a yellow oil. ${ }^{1} \mathrm{H}$ NMR (400 MHz, $\left.\mathrm{CDCl}_{3} / \mathrm{TMS}\right) \delta 7.93(\mathrm{~d}, 1 \mathrm{H}, J=1.9 \mathrm{~Hz}), 7.82(\mathrm{~d}, 2 \mathrm{H}, J=7.6 \mathrm{~Hz}), 7.73$ $(\mathrm{dd}, 1 \mathrm{H}, J=2.0,8.3 \mathrm{~Hz}), 7.62(\mathrm{t}, 1 \mathrm{H}, J=7.3 \mathrm{~Hz}), 7.51(\mathrm{t}, 2 \mathrm{H}, J=7.6 \mathrm{~Hz}), 7.36(\mathrm{~d}, 1 \mathrm{H}, J=8.3 \mathrm{~Hz})$, 7.27-7.22 (m, 2H), $6.88(\mathrm{t}, 1 \mathrm{H}, J=7.6 \mathrm{~Hz}), 6.77(\mathrm{~d}, 2 \mathrm{H}, J=7.8 \mathrm{~Hz}), 3.34(\mathrm{~s}, 3 \mathrm{H}) .{ }^{13} \mathrm{C} \mathrm{NMR}(150 \mathrm{MHz}$, Acetone- $\left.d_{6}\right) \delta 40.1,116.7,120.4,129.0,129.3,129.8,130.4,130.9,132.4,133.1,133.4,136.2,138.2$, 149.2, 150.3, 194.4. LRMS (EI) $\mathrm{m} / z$ : $321\left(\mathrm{M}^{+}\right)$. HRMS Calcd. for $\mathrm{C}_{20} \mathrm{H}_{16} \mathrm{ClNO}$ : 321.0920 , found: 321.0897. IR (neat):3062, 2882, 2818, 1653, 1586, 1496, 1276, 1133, 1045, 952, $712 \mathrm{~cm}^{-1}$.<smiles>O=C(c1ccccc1)c1ccc(N2CCc3ccccc32)c(Br)c1</smiles>

(3eg).According to the general procedure analogous to that described for $\mathbf{3 a a}$, except that the reaction was conducted at room temperature and crude material was purified by column chromatography on silica gel (hexane:AcOEt = 20:1), $\mathbf{3 e g}(45.2 \mathrm{mg}, 0.120 \mathrm{mmol}, 59 \%)$ was obtained from $1 \mathrm{e}(59.3 \mathrm{mg}$, $0.204 \mathrm{mmol}$ ) as a yellow oil. ${ }^{1} \mathrm{H}$ NMR (400 MHz, $\left.\mathrm{CDCl}_{3} / \mathrm{TMS}\right) \delta 8.13(\mathrm{~d}, 1 \mathrm{H}, J=2.0 \mathrm{~Hz}), 7.80(\mathrm{~d}$, $2 \mathrm{H}, J=7.3 \mathrm{~Hz}), 7.75(\mathrm{dd}, 1 \mathrm{H}, J=1.7,8.5 \mathrm{~Hz}), 7.61(\mathrm{t}, 1 \mathrm{H}, J=7.3 \mathrm{~Hz}), 7.54-7.48(\mathrm{~m}, 3 \mathrm{H}), 7.22(\mathrm{~d}$, $1 \mathrm{H}, J=7.3 \mathrm{~Hz}), 7.07$ (t, 1H, $J=7.8 \mathrm{~Hz}), 6.83(\mathrm{t}, 1 \mathrm{H}, J=7.6 \mathrm{~Hz}), 6.64(\mathrm{~d}, 1 \mathrm{H}, J=7.8 \mathrm{~Hz}), 4.08-3.98$ $(\mathrm{m}, 2 \mathrm{H}), 3.20(\mathrm{t}, 2 \mathrm{H}, J=8.3 \mathrm{~Hz}) .{ }^{13} \mathrm{C} \mathrm{NMR}\left(100 \mathrm{MHz}, \mathrm{CDCl}_{3}\right) \delta 29.0,54.3,110.7,119.5,120.0,123.5$, $125.1,126.9,128.4,129.8,130.3,131.1,132.5,134.7,136.4,137.3,147.8,148.6,194.2$. LRMS (EI) m/z: $378\left(\mathrm{M}^{+}\right)$. HRMS Calcd. for $\mathrm{C}_{21} \mathrm{H}_{16} \mathrm{BrNO}$ : 377.0415, found: 377.0398. IR (neat): 3386, 3065, $2850,1653,1584,1482,1280,1248,949,735 \mathrm{~cm}^{-1}$.<smiles>CC(C)(C)C(=O)c1ccc(N2CCc3ccccc32)cc1</smiles>

1-(4-(Indolin-1-yl)phenyl)-2,2-dimethylpropan-1-one (3fg). According to the general procedure analogous to that described for 3aa, except that the crude material was purified by $\mathrm{GPC}\left(\mathrm{CHCl}_{3}\right), \mathbf{3 f g}$ 
(49.0 mg, $0.175 \mathrm{mmol}, 89 \%)$ was obtained from $\mathbf{1 f}\left(38.0 \mathrm{mg}, 0.198 \mathrm{mmol}\right.$ ) as a white solid: $\mathrm{Mp} 78^{\circ} \mathrm{C}$ $\left(\mathrm{CH}_{2} \mathrm{Cl}_{2} /\right.$ hexane). ${ }^{1} \mathrm{H}$ NMR (400 MHz, $\left.\mathrm{CDCl}_{3} / \mathrm{TMS}\right) \delta 7.93$ (d, 2H, $J=8.8 \mathrm{~Hz}$ ), 7.29 (d, $1 \mathrm{H}, J=7.8$ $\mathrm{Hz}), 7.22-7.17(\mathrm{~m}, 3 \mathrm{H}), 7.13(\mathrm{t}, 1 \mathrm{H}, J=7.6 \mathrm{~Hz}), 6.84(\mathrm{t}, 1 \mathrm{H}, J=7.3 \mathrm{~Hz}), 4.02(\mathrm{t}, 2 \mathrm{H}, J=8.5 \mathrm{~Hz}), 3.17$ $(\mathrm{t}, 2 \mathrm{H}, J=8.5 \mathrm{~Hz}), 1.41(\mathrm{~s}, 9 \mathrm{H}) .{ }^{13} \mathrm{C} \mathrm{NMR}\left(100 \mathrm{MHz}, \mathrm{CDCl}_{3}\right) \delta 28.0,28.5,43.7,51.7,109.5,115.0$, 120.2, 125.2, 127.1, 128.4, 130.8, 131.9, 145.4, 146.6, 205.4. LRMS (EI) m/z: $279\left(\mathrm{M}^{+}\right)$. HRMS Calcd. for $\mathrm{C}_{19} \mathrm{H}_{21} \mathrm{NO}: 279.1623$, found: 279.1627. IR (neat): 2969, 2871, 1649, 1587, 1511, 1481, 1387, 1278 , $1165,959,835 \mathrm{~cm}^{-1}$.<smiles>CCCC(=O)c1ccc(N2CCc3ccccc32)cc1</smiles>

1-(4-(Indolin-1-yl)phenyl)-2-methylpropan-1-one (3gg). According to the general procedure analogous to that described for 3aa, except that the crude material was purified by column chromatography on silica gel (hexane:AcOEt = 10:1), $3 g g(47.1 \mathrm{mg}, 0.177 \mathrm{mmol}, 89 \%)$ was obtained from $1 \mathrm{~g}$ (35.4 mg, $0.199 \mathrm{mmol})$ as a brown oil. ${ }^{1} \mathrm{H} \mathrm{NMR}\left(400 \mathrm{MHz}, \mathrm{CDCl}_{3} / \mathrm{TMS}\right) \delta 7.98(\mathrm{~d}, 2 \mathrm{H}, J=$ $8.8 \mathrm{~Hz}), 7.31(\mathrm{~d}, 1 \mathrm{H}, J=7.8 \mathrm{~Hz}), 7.25-7.20(\mathrm{~m}, 3 \mathrm{H}), 7.14(\mathrm{t}, 1 \mathrm{H}, J=7.6 \mathrm{~Hz}), 6.85(\mathrm{t}, 1 \mathrm{H}, J=7.3 \mathrm{~Hz})$, $4.04(\mathrm{t}, 2 \mathrm{H}, J=8.5 \mathrm{~Hz}), 3.54(\mathrm{sept}, 1 \mathrm{H}, J=6.7 \mathrm{~Hz}), 3.17(\mathrm{t}, 2 \mathrm{H}, J=8.3 \mathrm{~Hz}), 1.23(\mathrm{~d}, 6 \mathrm{H}, J=6.8 \mathrm{~Hz})$.

${ }^{13} \mathrm{C} \mathrm{NMR}\left(100 \mathrm{MHz}, \mathrm{CDCl}_{3}\right) \delta 19.4,28.0,34.7,51.7,109.7,115.3,120.4 .125 .3,127.1,127.6,130.1$, 132.0, 145.1, 147.7, 202.6. LRMS (EI) $m / z: 265\left(\mathrm{M}^{+}\right)$. HRMS Calcd. for $\mathrm{C}_{18} \mathrm{H}_{19} \mathrm{NO}: 265.1467$, found: 265.1443. IR (neat): 2969, 2870, 1665, 1587, 1481, 1383, 1229, 1158, 979, $742 \mathrm{~cm}^{-1}$.<smiles>CN(c1ccccc1)c1ccc2c(c1)CC(C)(C)C2=O</smiles>

2,2-Dimethyl-5-(methyl(phenyl)amino)-2,3-dihydro-1H-inden-1-one (3ha). According to the general procedure analogous to that described for 3aa, $3 \mathbf{h a ~}(46.8 \mathrm{mg}, 0.176 \mathrm{mmol}, 88 \%)$ was obtained from $1 \mathbf{h}(38.1 \mathrm{mg}, 0.200 \mathrm{mmol})$ as a yellow oil. ${ }^{1} \mathrm{H} \mathrm{NMR}\left(400 \mathrm{MHz}, \mathrm{CDCl}_{3} / \mathrm{TMS}\right) \delta 7.56(\mathrm{~d}, 1 \mathrm{H}, J=$ $8.8 \mathrm{~Hz}), 7.43(\mathrm{t}, 2 \mathrm{H}, J=7.8 \mathrm{~Hz}), 7.28-7.21(\mathrm{~m}, 3 \mathrm{H}), 6.71(\mathrm{dd}, 1 \mathrm{H}, J=1.9,8.8 \mathrm{~Hz}), 6.64(\mathrm{~s}, 1 \mathrm{H}), 3.39$ $(\mathrm{s}, 3 \mathrm{H}), 2.86(\mathrm{~s}, 2 \mathrm{H}), 1.21(\mathrm{~s}, 6 \mathrm{H}) .{ }^{13} \mathrm{C} \mathrm{NMR}\left(100 \mathrm{MHz}, \mathrm{CDCl}_{3}\right) \delta 25.5,40.5,43.0,45.4,109.3,114.4$, 125.2, 125.5, 125.9, 126.4, 129.9, 147.3, 154.57, 154.59, 209.1. LRMS (EI) $m / z: 265\left(\mathrm{M}^{+}\right)$. HRMS Calcd. for $\mathrm{C}_{18} \mathrm{H}_{19} \mathrm{NO}$ : 265.1467, found: 265.1441. IR (neat): 2957, 2921, 1689, 1603, 1584, 1494, $1355,1296,1097,769 \mathrm{~cm}^{-1}$. 
<smiles>CN(c1ccccc1)c1cnccn1</smiles>

$N$-Methyl- $N$-phenylpyrazin-2-amine (3ja). According to the general procedure analogous to that described for 3aa, except that the reaction was conducted in cyclohexane at room temperature and crude material was purified by column chromatography on silica gel (hexane:AcOEt = 10:1), 3ja (26.2 $\mathrm{mg}, 0.141 \mathrm{mmol}, 71 \%)$ was obtained from $\mathbf{1 j}(22.0 \mathrm{mg}, 0.200 \mathrm{mmol})$ as a brown oil. ${ }^{1} \mathrm{H}$ NMR (400 $\left.\mathrm{MHz}, \mathrm{CDCl}_{3} / \mathrm{TMS}\right) \delta 8.10(\mathrm{~s}, 1 \mathrm{H}), 7.94(\mathrm{~s}, 1 \mathrm{H}), 7.83(\mathrm{~d}, 1 \mathrm{H}, J=2.4 \mathrm{~Hz}), 7.45(\mathrm{t}, 2 \mathrm{H}, J=7.8 \mathrm{~Hz})$, 7.31-7.26 (m, 3H), $3.46(\mathrm{~s}, 3 \mathrm{H}) .{ }^{13} \mathrm{C} \mathrm{NMR}\left(100 \mathrm{MHz}, \mathrm{CDCl}_{3}\right) \delta 38.2,126.3,126.5,130.0,132.6,132.9$, 141.4, 145.1, 154.8. LRMS (EI) $m / z$ : $185\left(\mathrm{M}^{+}\right)$. HRMS Calcd. for $\mathrm{C}_{11} \mathrm{H}_{11} \mathrm{~N}_{3}$ : 185.0953, found: 185.0953. IR (neat): 3056, 2927, 1572, 1516, 1496, 1401, 1152, 995, 825, $768 \mathrm{~cm}^{-1}$. The spectra data matched those reported in the literature. ${ }^{17}$<smiles>CN(c1ccccc1)c1ccc2ccccc2n1</smiles>

$\boldsymbol{N}$-Methyl- $\boldsymbol{N}$-phenylquinolin-2-amine (3ka). According to the general procedure analogous to that described for 3aa, except that the reaction was conducted at $90{ }^{\circ} \mathrm{C}$, $\mathbf{3 k a}(36.7 \mathrm{mg}, 0.157 \mathrm{mmol}, 78 \%)$ was obtained from $1 \mathrm{k}(31.8 \mathrm{mg}, 0.200 \mathrm{mmol})$ as a colorless oil. ${ }^{1} \mathrm{H}$ NMR (400 $\left.\mathrm{MHz}, \mathrm{CDCl}_{3} / \mathrm{TMS}\right) \delta$ $7.79(\mathrm{~d}, 1 \mathrm{H}, J=8.3 \mathrm{~Hz}), 7.68(\mathrm{~d}, 1 \mathrm{H}, J=9.3 \mathrm{~Hz}), 7.60-7.52(\mathrm{~m}, 2 \mathrm{H}), 7.46-7.40(\mathrm{~m}, 2 \mathrm{H}), 7.32-7.27(\mathrm{~m}$, $2 \mathrm{H}), 7.27-7.21(\mathrm{~m}, 2 \mathrm{H}), 6.74(\mathrm{~d}, 1 \mathrm{H}, J=9.3 \mathrm{~Hz}), 3.63(\mathrm{~s}, 3 \mathrm{H}) .{ }^{13} \mathrm{C} \mathrm{NMR}\left(150 \mathrm{MHz}, \mathrm{C}_{6} \mathrm{D}_{6}\right) \delta 38.8$, 112.2 , 122.7, 123.9, 125.8, 126.8, 127.56, 127.63, 129.7, 129.9, 136.3, 147.1, 148.8, 157.2. LRMS (EI) $m / z: 234\left(\mathrm{M}^{+}\right)$. HRMS Calcd. for $\mathrm{C}_{16} \mathrm{H}_{14} \mathrm{~N}_{2}$ : 234.1157, found: 234.1131. IR (neat): 3058, 2921, $1617,1593,1558,1495,1483,1384,1114,817 \mathrm{~cm}^{-1}$. The spectra data matched those reported in the literature. $^{17 \mathrm{a}}$<smiles>CN(c1ccccc1)c1ccc2cc(Br)ccc2n1</smiles>

6-Bromo- $N$-methyl- $N$-phenylquinolin-2-amine (3la). According to the general procedure analogous to that described for 3aa, except that the reaction was conducted in cyclohexane at room temperature, 3la (53.1 mg, $0.170 \mathrm{mmol}, 85 \%)$ was obtained from 11 (47.6 mg, $0.200 \mathrm{mmol}$ ) as a white solid: $\mathrm{Mp}$ $120{ }^{\circ} \mathrm{C}$ (hexane). ${ }^{1} \mathrm{H}$ NMR (400 MHz, $\left.\mathrm{CDCl}_{3} / \mathrm{TMS}\right) \delta 7.71(\mathrm{~d}, 1 \mathrm{H}, J=2.4 \mathrm{~Hz}), 7.68-7.55(\mathrm{~m}, 3 \mathrm{H})$, $7.44(\mathrm{t}, 2 \mathrm{H}, J=8.0 \mathrm{~Hz}), 7.30-7.26(\mathrm{~m}, 3 \mathrm{H}), 6.73(\mathrm{~d}, 1 \mathrm{H}, J=9.3 \mathrm{~Hz}), 3.61(\mathrm{~s}, 3 \mathrm{H}) .{ }^{13} \mathrm{C} \mathrm{NMR}(100 \mathrm{MHz}$, $\left.\mathrm{CDCl}_{3}\right) \delta 38.6,112.7,115.1,124.5,126.2,126.7,128.4,129.2,129.9,132.5,135.2,146.1,146.6,157.1$. 
LRMS (EI) $m / z: 313\left(\mathrm{M}^{+}\right)$. HRMS Calcd. for $\mathrm{C}_{16} \mathrm{H}_{13} \mathrm{BrN}_{2}$ : 312.0262 , found: 312.0247. IR (neat): 2924 , 2853, 1957, 1900, 1613, 1486, 1384, 1063, 815, $707 \mathrm{~cm}^{-1}$.<smiles>c1ccc2c(c1)CCN2c1nccc2ccccc12</smiles>

1-(Indolin-1-yl)isoquinoline (3mg). According to the general procedure analogous to that described for 3aa, except that the crude material was purified by column chromatography on silica gel (hexane:AcOEt = 10:1), $\mathbf{3 m g}$ (45.3 mg, $0.184 \mathrm{mmol}, 92 \%)$ was obtained from $1 \mathbf{m}$ (30.9 mg, 0.194 mmol) as brown solid: $\mathrm{Mp} 83{ }^{\circ} \mathrm{C}\left(\mathrm{CH}_{2} \mathrm{Cl}_{2} /\right.$ hexane). ${ }^{1} \mathrm{H} \mathrm{NMR}\left(400 \mathrm{MHz}, \mathrm{CDCl}_{3} / \mathrm{TMS}\right) \delta 8.26(\mathrm{~d}, 1 \mathrm{H}$, $J=5.9 \mathrm{~Hz}), 8.09$ (d, 1H, $J=8.8 \mathrm{~Hz}), 7.82(\mathrm{~d}, 1 \mathrm{H}, J=8.3 \mathrm{~Hz}), 7.67$ (t, 1H, $J=7.6 \mathrm{~Hz}), 7.49$ (t, 1H, $J$ $=7.8 \mathrm{~Hz}), 7.39(\mathrm{~d}, 1 \mathrm{H}, J=5.8 \mathrm{~Hz}), 7.26-7.22(\mathrm{~m} \mathrm{1H}), 6.94(\mathrm{t}, 1 \mathrm{H}, J=7.8 \mathrm{~Hz}), 6.81(\mathrm{t}, 1 \mathrm{H}, J=7.6 \mathrm{~Hz})$, $6.48(\mathrm{~d}, 1 \mathrm{H}, J=7.8 \mathrm{~Hz}), 4.27(\mathrm{t}, 2 \mathrm{H}, J=8.1 \mathrm{~Hz}), 3.20(\mathrm{t}, 2 \mathrm{H}, J=8.3 \mathrm{~Hz}) .{ }^{13} \mathrm{C} \mathrm{NMR}\left(100 \mathrm{MHz}, \mathrm{CDCl}_{3}\right)$ $\delta 28.6,53.2,110.6,117.0,120.0,122.4,124.8,125.9,126.4,126.5,126.9,130.1,131.9,138.0,141.2$, 148.3, 155.3. LRMS (EI) $m / z: 246\left(\mathrm{M}^{+}\right)$. HRMS Calcd. for $\mathrm{C}_{17} \mathrm{H}_{14} \mathrm{~N}_{2}: 246.1157$, found: 246.1150. IR (neat): $3050,2978,2919,2871,1606,1555,1478,1399,1225,812 \mathrm{~cm}^{-1}$.<smiles>CN(C)C(=O)c1ccc(N(C)c2ccccc2)nc1</smiles>

$N, N$-Dimethyl-6-(methyl(phenyl)amino)nicotinamide (3na). According to the general procedure analogous to that described for 3aa, except that the crude material was purified by column chromatography on silica gel (hexane:AcOEt =1:2), 3na $(41.1 \mathrm{mg}, 0.161 \mathrm{mmol}, 83 \%)$ was obtained from $1 \mathrm{n}(35.1 \mathrm{mg}, 0.195 \mathrm{mmol})$ as a colorless oil. ${ }^{1} \mathrm{H} \mathrm{NMR}\left(400 \mathrm{MHz}, \mathrm{CDCl}_{3} / \mathrm{TMS}\right) \delta 8.36(\mathrm{~d}, 1 \mathrm{H}, J$ $=2.4 \mathrm{~Hz}), 7.48-7.40(\mathrm{~m}, 3 \mathrm{H}), 7.30-7.25(\mathrm{~m}, 3 \mathrm{H}), 6.47(\mathrm{~d}, 1 \mathrm{H}, J=8.8 \mathrm{~Hz}), 3.50(\mathrm{~s}, 3 \mathrm{H}), 3.09(\mathrm{~s}, 6 \mathrm{H})$. ${ }^{13} \mathrm{C}$ NMR $\left(100 \mathrm{MHz}, \mathrm{CDCl}_{3}\right) \delta 16.5,38.5,107.8,120.5,126.2,126.6,129.8,136.6,145.9,147.5$, 159.1, 170.0. LRMS (EI) m/z: $255\left(\mathrm{M}^{+}\right)$. HRMS Calcd. for $\mathrm{C}_{15} \mathrm{H}_{17} \mathrm{~N}_{3} \mathrm{O}: 255.1372$, found: 255.1345 . IR (neat): 3496, 2922, 1623, 1605, 1589, 1481, 1373, 1264, 1124, $773 \mathrm{~cm}^{-1}$.<smiles>c1ccc(N2Cc3ccccc3-c3ccccc32)cc1</smiles>

5-Phenyl-5,6-dihydrophenanthridine (5a). In a glove box under an Ar atomosphere, to a mixture of 4a $(57.1 \mathrm{mg}, 0.197 \mathrm{mmol})$ in 1,4 -dioxane $(0.7 \mathrm{~mL})$ were added $t$-Bu-P4 solution $(50 \mu \mathrm{L}, 0.8 \mathrm{M}$ in 
hexane, $0.04 \mathrm{mmol})$ and $5 \AA$ molecular sieves $(100 \mathrm{mg})$ in an oven-dried vial equipped with a stirrer bar. The vial was sealed with a cap containing an inner Teflon film, and taken outside the glove box. After stirring at $120^{\circ} \mathrm{C}$ for $18 \mathrm{~h}$, saturated $\mathrm{NH}_{4} \mathrm{Cl}$ aqueous solution $(1 \mathrm{~mL})$ was added to the reaction mixture at $0{ }^{\circ} \mathrm{C}$. The mixture was extracted with AcOEt $(10 \mathrm{~mL} \times 3)$. The combined organic layer was collected, washed with brine $(10 \mathrm{~mL})$, dried over $\mathrm{Na}_{2} \mathrm{SO}_{4}$, and concentrated. The crude material was purified by column chromatography on silica gel (hexane:AcOEt =10:1) to afford 5a (37.1 mg, 0.144 mmol, $73 \%$ ) as a white solid: $\mathrm{Mp} 135-137^{\circ} \mathrm{C}\left(\mathrm{CH}_{2} \mathrm{Cl}_{2} /\right.$ hexane). ${ }^{1} \mathrm{H}$ NMR (400 $\left.\mathrm{MHz}, \mathrm{CDCl}_{3} / \mathrm{TMS}\right) \delta$ $7.78(\mathrm{~d}, 2 \mathrm{H}, J=7.8 \mathrm{~Hz}), 7.39-7.33(\mathrm{~m}, 3 \mathrm{H}), 7.28-7.22(\mathrm{~m}, 3 \mathrm{H}), 7.14$ (d, 1H, J=7.3 Hz), 7.09 (t, 2H, $J=6.8 \mathrm{~Hz}), 6.95(\mathrm{t}, 2 \mathrm{H}, J=8.8 \mathrm{~Hz}), 4.72(\mathrm{~s}, 2 \mathrm{H}) .{ }^{13} \mathrm{C} \mathrm{NMR}\left(150 \mathrm{MHz}, \mathrm{CDCl}_{3}\right) \delta 52.5,116.6,120.3$, 122.6, 122.7, 123.2, 124.0, 125.0, 125.6, 127.2, 127.7, 128.4, 129.2, 131.9, 133.1, 144.5, 146.2. LRMS (EI) $m / z$ : $257\left(\mathrm{M}^{+}\right)$. HRMS Calcd. for $\mathrm{C}_{19} \mathrm{H}_{15} \mathrm{~N}$ : 257.1205, found: 257.1231. IR (neat): 3059, 1593, $1491,1439,1270,1107,884,764,746,724 \mathrm{~cm}^{-1}$. The spectra data matched those reported in the literature. $^{18}$<smiles>CC1(C)CN(c2ccccc2)c2ccccc2-c2ccccc21</smiles>

7,7-Dimethyl-5-phenyl-6,7-dihydro-5H-dibenzo[b,d]azepine (5b). According to the general procedure analogous to that described for 5a, except that the reaction was conducted in DMI at $150{ }^{\circ} \mathrm{C}$, 5b (44.5 mg, $0.149 \mathrm{mmol}, 74 \%)$ was obtained from 4b (66.5 mg, $0.201 \mathrm{mmol})$ as a white solid: Mp $88^{\circ} \mathrm{C}\left(\mathrm{CH}_{2} \mathrm{Cl}_{2} /\right.$ hexane). ${ }^{1} \mathrm{H}$ NMR (400 MHz, $\left.\mathrm{CDCl}_{3} / \mathrm{TMS}\right) \delta 7.45-7.40(\mathrm{~m}, 3 \mathrm{H}), 7.35-7.24(\mathrm{~m}, 4 \mathrm{H})$, 7.15-7.12 (m, 1H), 7.12-7.06 (m, 2H), 6.74-6.68 (m, 3H), $3.78(\mathrm{~s}, 2 \mathrm{H}), 1.21(\mathrm{~s}, 6 \mathrm{H}) .{ }^{13} \mathrm{C}$ NMR $(150$ MHz, DMSO- $\left.d_{6}\right) \delta 28.7,38.1,67.9,115.9,118.3,125.0,126.1,126.79,126.85,127.5,128.5,128.7$, 129.2, 129.3, 138.8, 140.7, 144.5, 144.6, 149.2. LRMS (EI) $\mathrm{m} / z: 299\left(\mathrm{M}^{+}\right)$. HRMS Calcd. for $\mathrm{C}_{22} \mathrm{H}_{21} \mathrm{~N}$ : 299.1674, found: 299.1690. IR (neat): 3057, 2962, 2870, 1592, 1495, 1436, 1319, 1275, 1159, $740 \mathrm{~cm}^{-}$ 1.

\section{Procedure of amination of 1a with 2a on a $1.0 \mathrm{mmol}$ scale (Table 1, entry 9).}

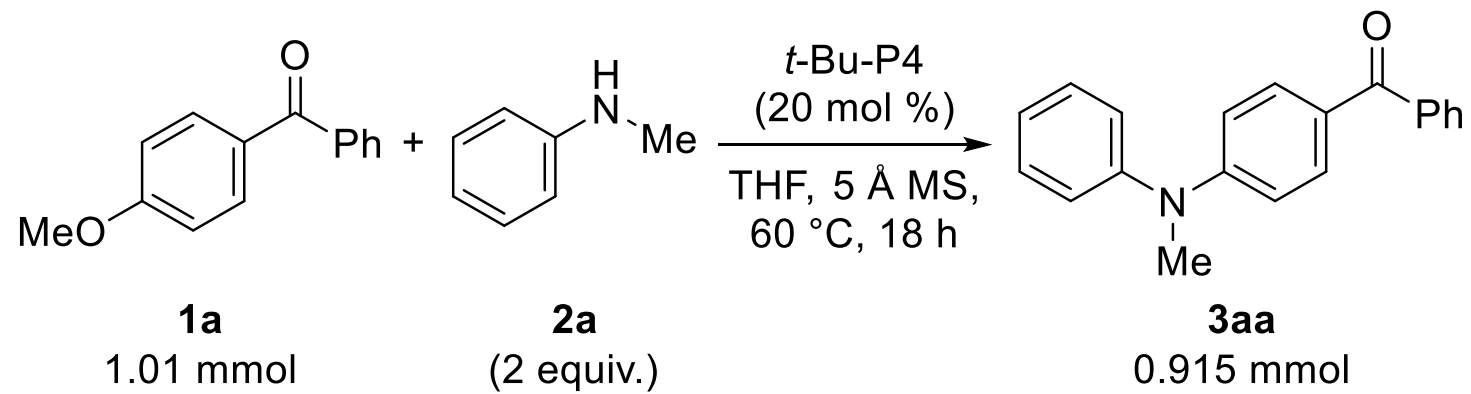

In a glove box under an Ar atmosphere, to a mixture of $\mathbf{1 a}(215.1 \mathrm{mg}, 1.01 \mathrm{mmol})$ and $\mathbf{2 a}(218.2 \mathrm{mg}$, 
$2.04 \mathrm{mmol})$ in THF (3.5 mL) were added $t$-Bu-P4 solution $(250 \mu \mathrm{L}, 0.8 \mathrm{M}$ in hexane, $0.2 \mathrm{mmol})$ and $5 \AA$ molecular sieves (500 mg) in an oven-dried vial equipped with a stirrer bar. The vial was sealed with a cap containing an inner Teflon film, and taken outside the glove box. After $60{ }^{\circ} \mathrm{C}$ for $18 \mathrm{~h}$, saturated $\mathrm{NH}_{4} \mathrm{Cl}$ aqueous solution $(5 \mathrm{~mL})$ was added to the reaction mixture at $0{ }^{\circ} \mathrm{C}$. The mixture was extracted with AcOEt (30 mL x 3). The combined organic layer was washed with brine (30 mL), dried over $\mathrm{Na}_{2} \mathrm{SO}_{4}$, and concentrated. The crude material was purified by column chromatography on silica gel (toluene) to afford 3aa (262.8 $\mathrm{mg}, 0.915 \mathrm{mmol}, 90 \%)$.

\section{References}

1. M. Shigeno, K. Hayashi, K. Nozawa-Kumada, Y. Kondo, Chem. Eur. J. 2019, 25, 6077.

2. Y. Shimizu, S. Izumi, J. Takenaka, J. Momoda, PCT Int. Appl. WO2013042800.

3. V. Zanirato, G. P. Pollini, C. De Risi, F. Valente, A. Melloni, S. Fusi, J. Barbetti, M. Olivuccci, Tetrahedron 2007, 63, 4975.

4. S. Broch, B. Aboab, F. Anizon, P. Moreau, Eur. J. Med. Chem. 2010, 45, 1657.

5. Y. Kim, J. Son, J. Kim, D.-J. Baek, Y. S. Lee, E. J. Lim, J. K. Lee, A. N. Pae, S.-J. Min, Y. S. Cho, Chem. Pharm. Bull. 2014, 62. 508.

6. Ł. Struk, J. G. Sośnicki, Synthesis 2012, 44, 735.

7. P. Pradeep, K. J. Ngwira, C. Reynolds, A. L. Rousseau, A. Lemmerer, M. A. Fernandes, M. M.

Johnson, C. B. de Koning, Tetrahedron 2016, 72, 8417.

8. S. A. Babu, M. Yasuda, A. Baba, Org. Lett. 2007, 9, 405.

9. (a) X. Quan, S. Kerdphon, P. G. Andersson, Chem. Eur. J. 2015, 21, 3576. (b) Y. Li, D. Xue, W. Lu, C. Wang, Z.-T. Liu, J. Xiao, Org. Lett, 2014, 16, 66.

10. (a) R. J. Lundgren, A. Sappong-Kumankumah, M. Stradiotto, Chem. Eur. J. 2010, 16, 1983. (b) B. L. P. Li, C. Fu, L. Xue, Z. Lin, S. Ma, Adv. Synth. Catal. 2011, 353, 100. (c) G. Manolikakes, A. Gavryushin, P. Knochel, J. Org. Chem. 2008, 73, 1429.

11. (a) H. Kim, S. Park, Y. Baek, K. Um, G. U. Han, D.-H. Jeon, S. H. Han, P. H. Lee, J. Org. Chem. 2018. 83. 3486. (b) K. Kamikawa, S. Sugimoto, M. Uemura, J. Org. Chem. 1998, 63, 8407.

12. J. de Jong, D. Heijnen, H. Helbert, B. L. Feringa, Chem. Commun. 2019, 55, 2908.

13. (a) S. Suárez-Pantiga, R. Hernández-Ruiz, C. Virumbrales, M. R. Pedrosa, R. Sanz, Angew. Chem. Int. Ed. 2019, 58, 2129. (b) P. Y. Choy, K. H. Chung, Q. Yang, C. M. So, R. W.-Y. Sun, F. Y. Kwong, Chem. Asian J. 2018, 13, 2465.

14. (a) C.-Y. Gao, L.-M. Yang, J. Org. Chem. 2008, 73, 1624. (b) K. Matsubara, K. Ueno, Y. Koga, K. Hara, J. Org. Chem. 2007, 72, 5069.

15. S. S. Bhojgude, T. Kaicharla, A. T. Biju, Org. Lett. 2013, 15, 5452.

16. (a) Q.-L. Shao, Z.-J. Jiang, W.-K. Su, Tetrahedron Lett. 2018, 59, 2277. (b) R. Kuwano, M. Utsnomiya, J. F. Hartwig, J. Org. Chem. 2002, 67, 6479. 
17. (a) X. Liu, H. Yue, J. Jia, L. Guo, M. Rueping, Chem. Eur. J. 2017, 23, 11771. (b) B. J. Tardiff, M. Stradiotto, Eur. J. Org. Chem. 2012, 3972.

18. J. J. Eisch, C. A. Kovacs, P. Chobe, J. Org. Chem. 1989, 54, 1275. 

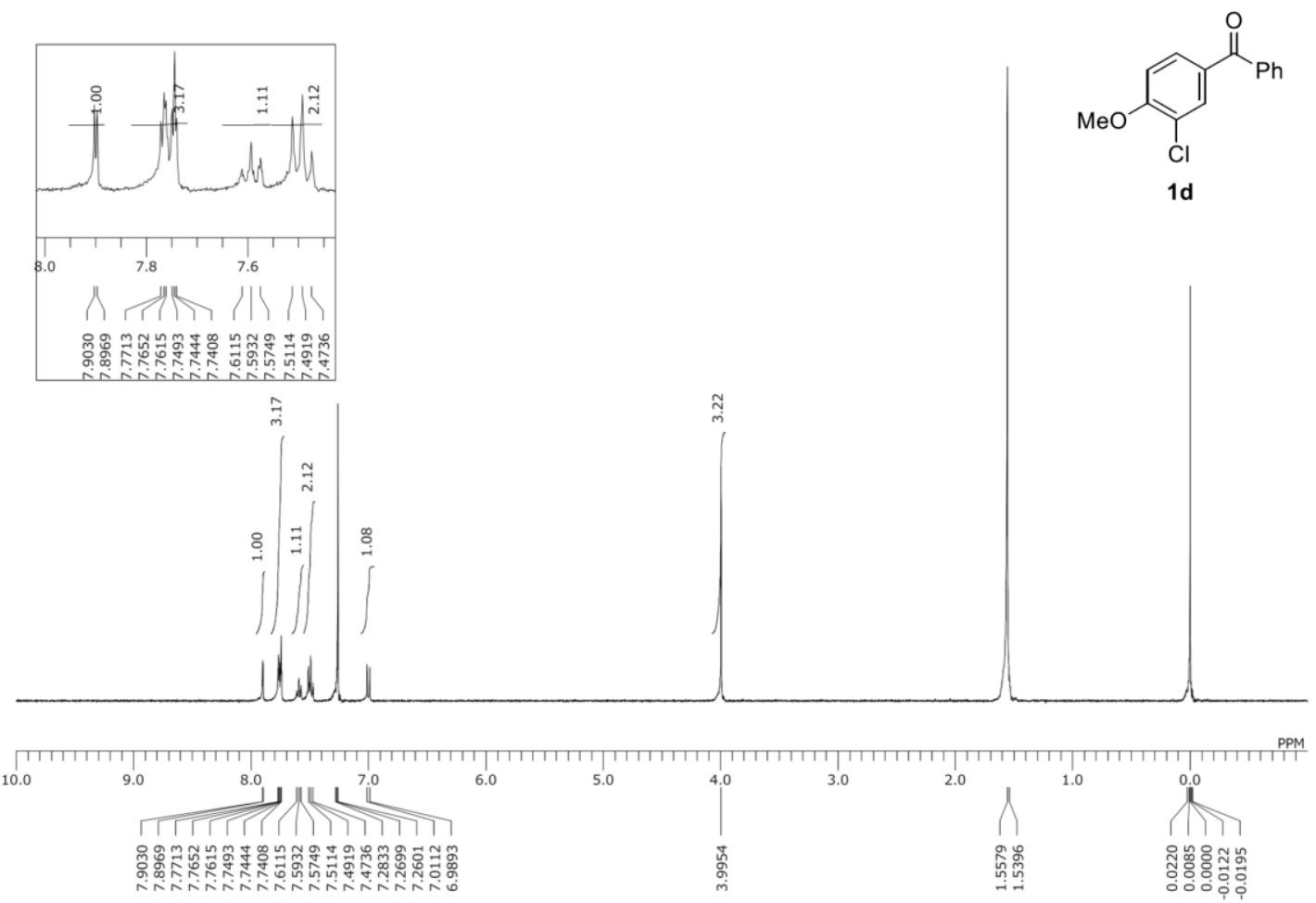

${ }^{13} \mathrm{C}$ NMR spectra of $\mathbf{1 d}$

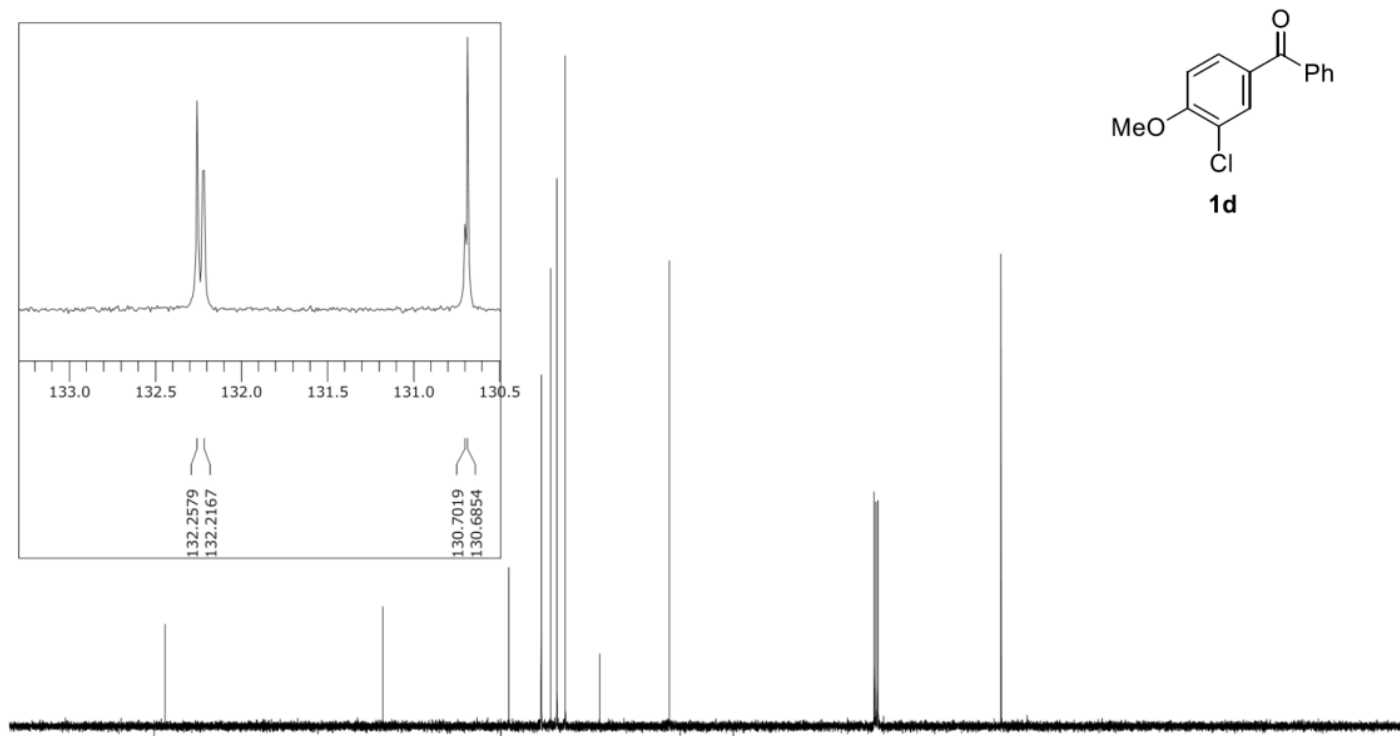

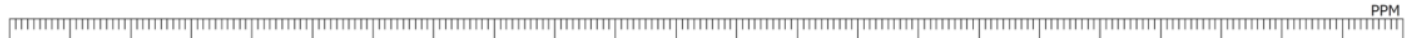

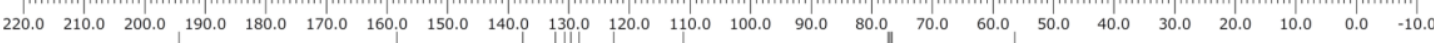

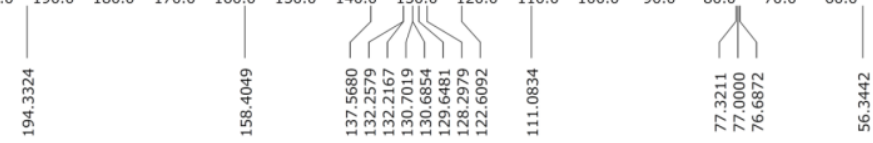


${ }^{1} \mathrm{H}$ NMR spectra of $\mathbf{1 g}$

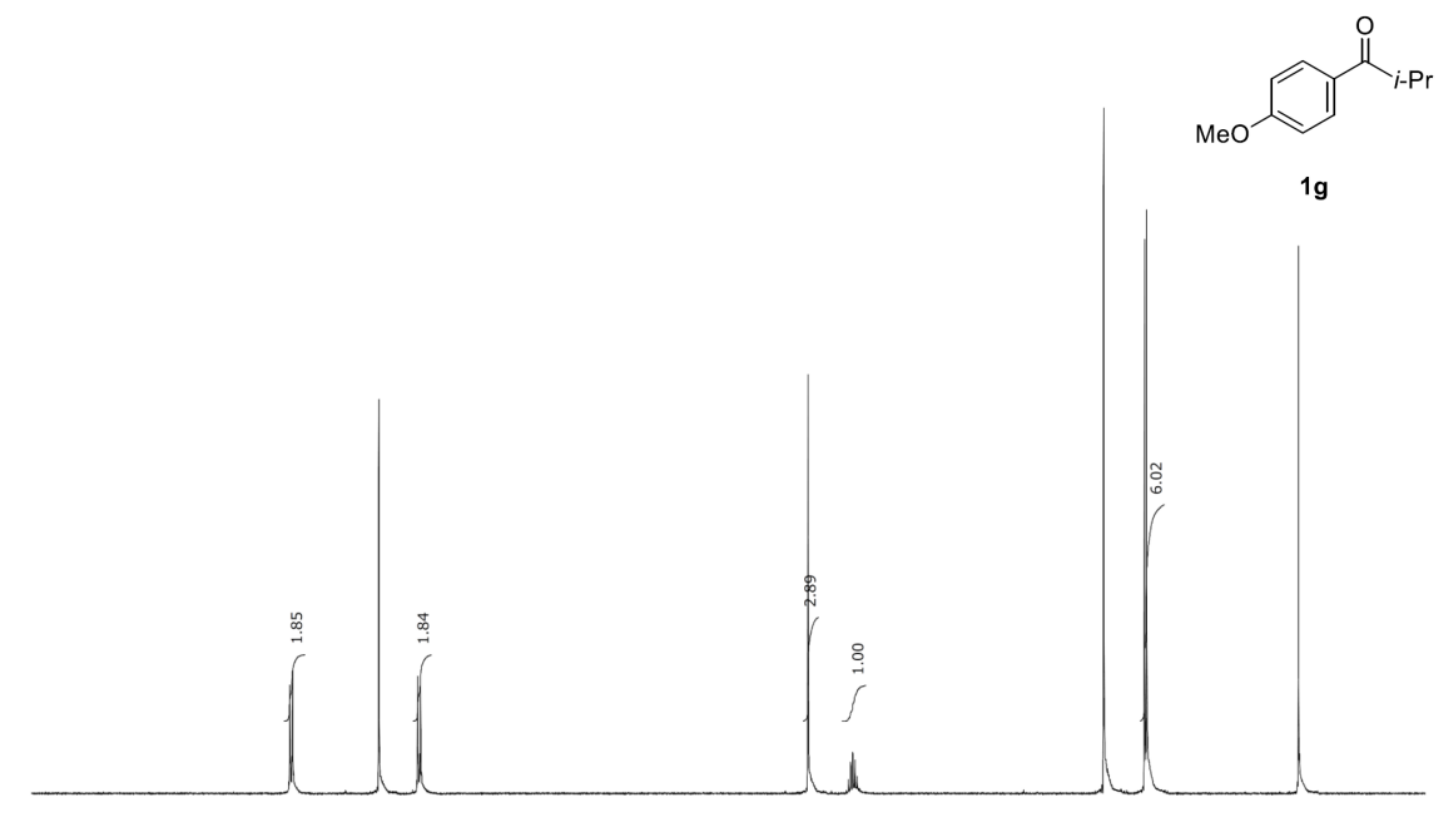

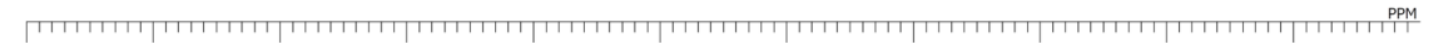
10.0 in

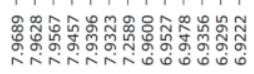

$6.0 \quad 5.0$

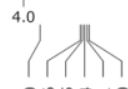

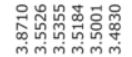

2.0

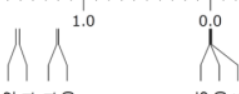

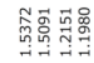

${ }^{13} \mathrm{C}$ NMR spectra of $\mathbf{1 g}$

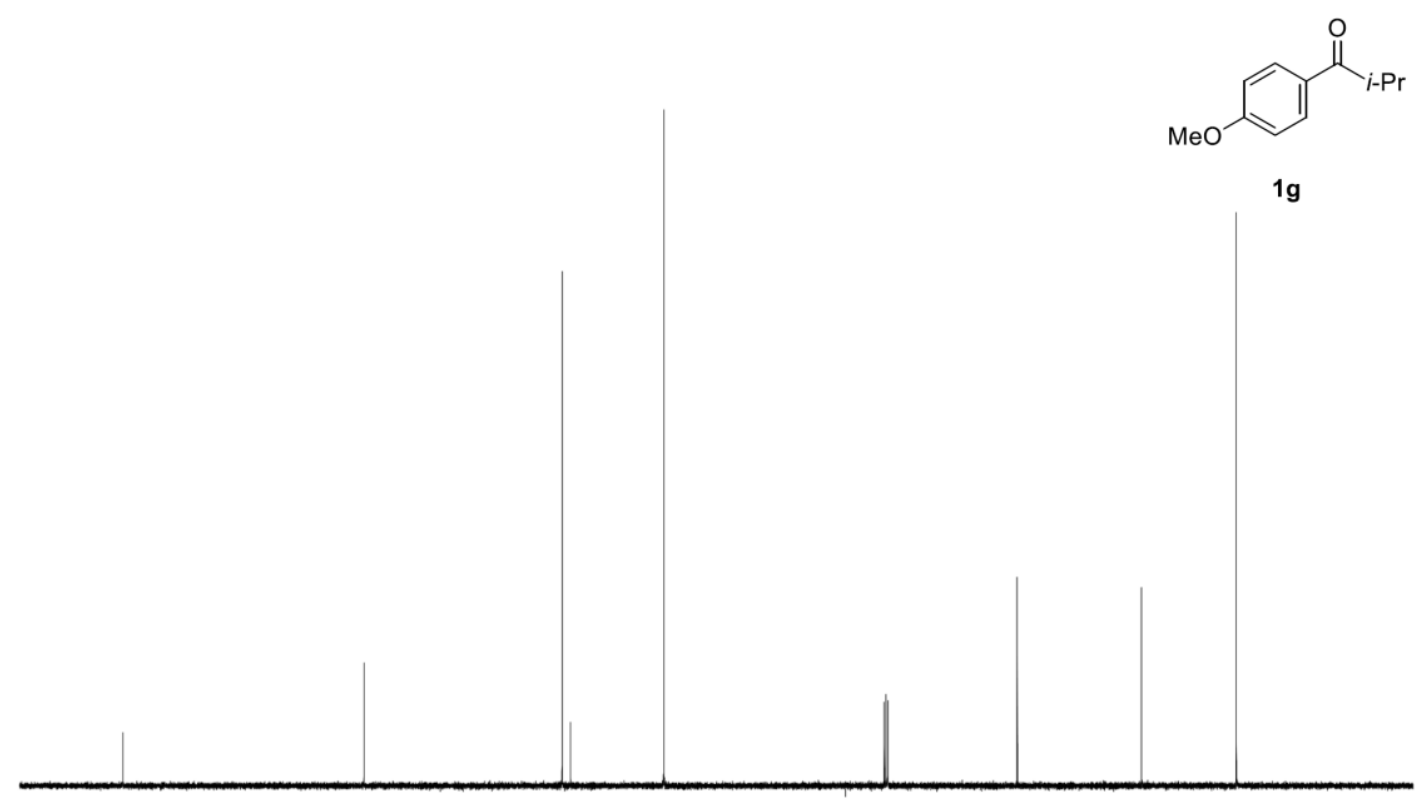

[

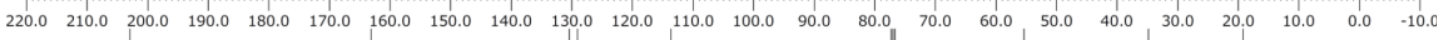
芯

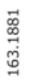

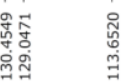

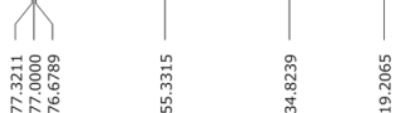


${ }^{1} \mathrm{H}$ NMR spectra of $\mathbf{1 I}$
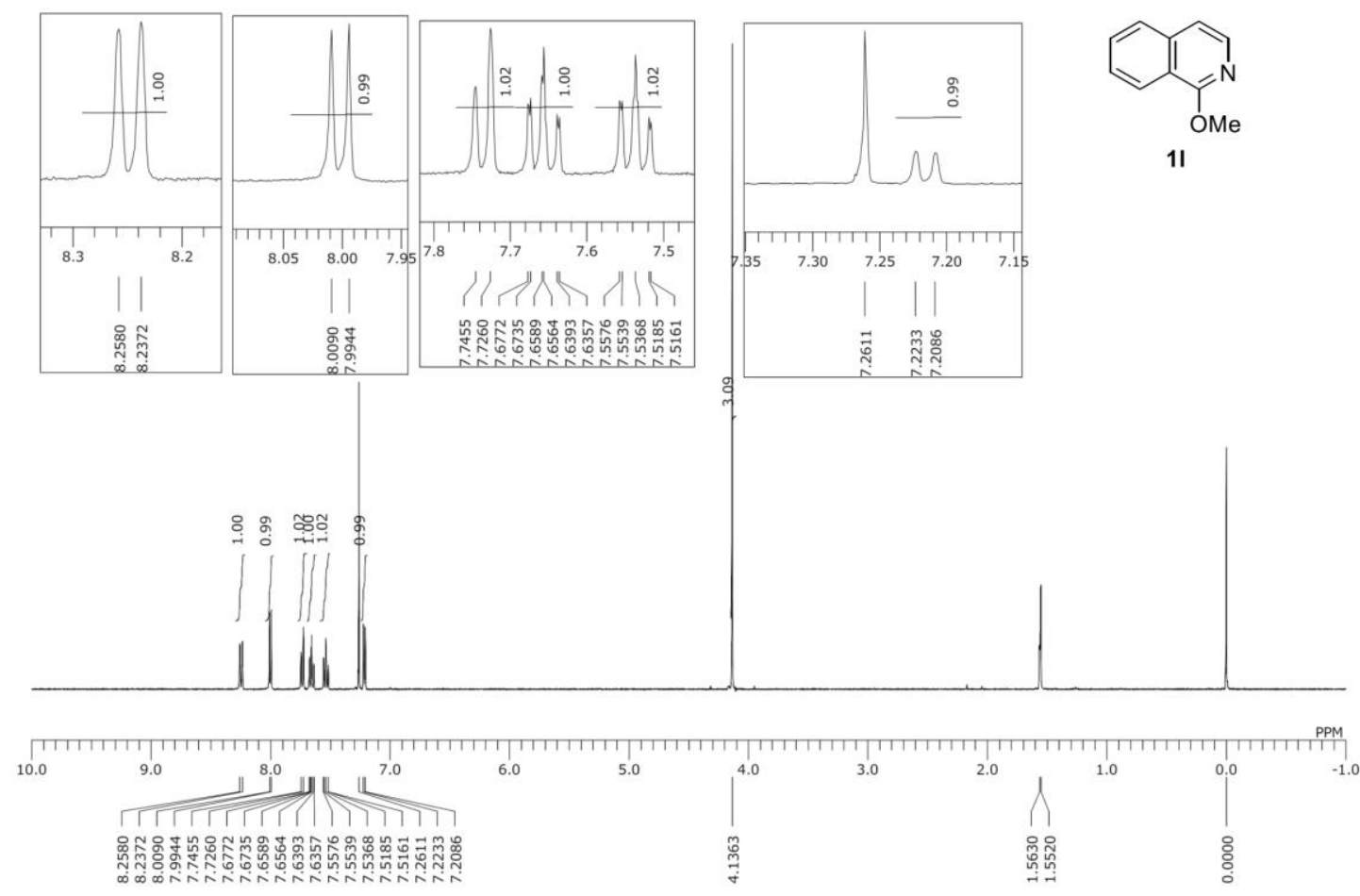

${ }^{13} \mathrm{C}$ NMR spectra of $\mathbf{1 1}$

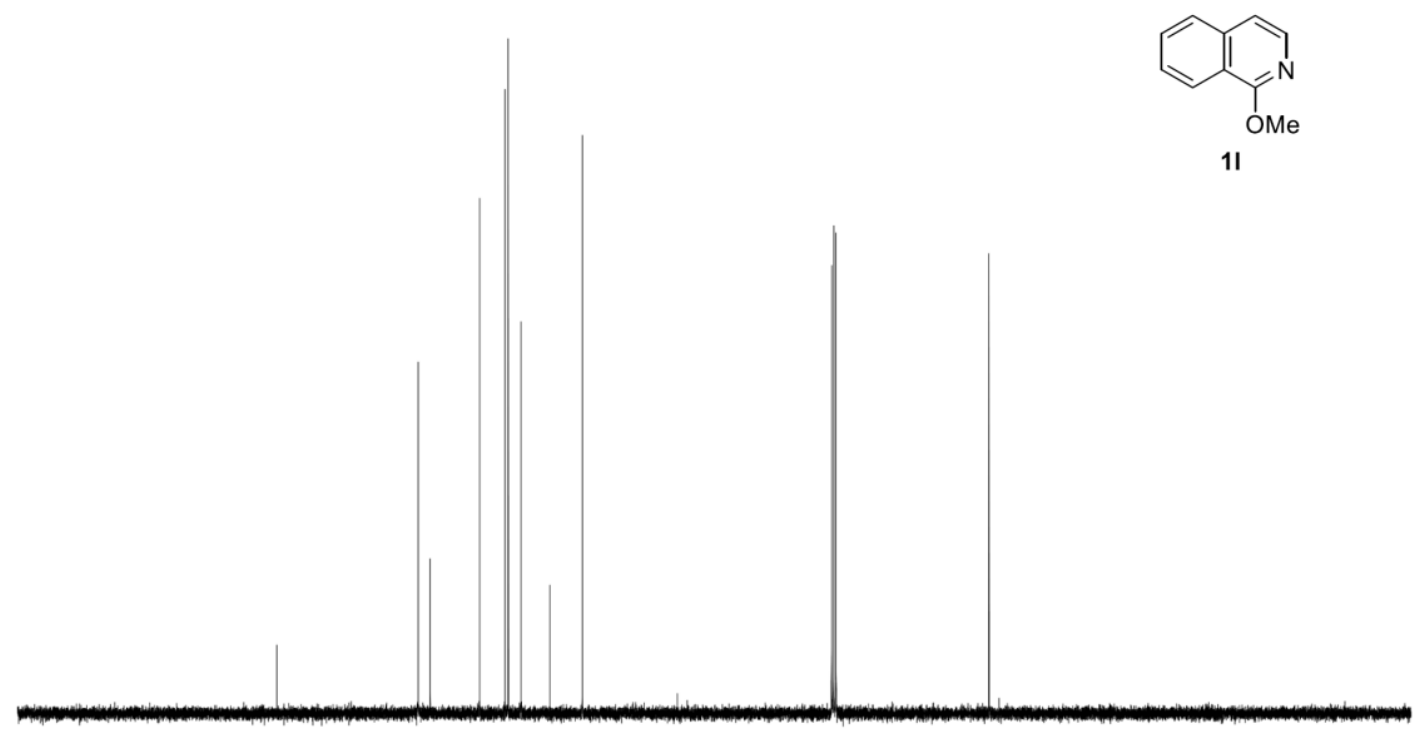

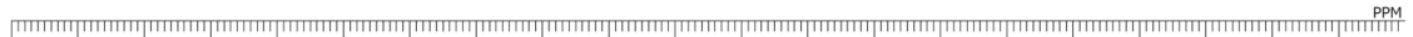

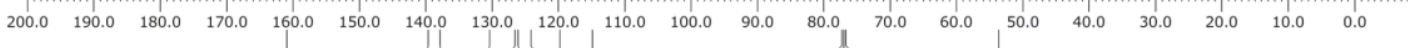

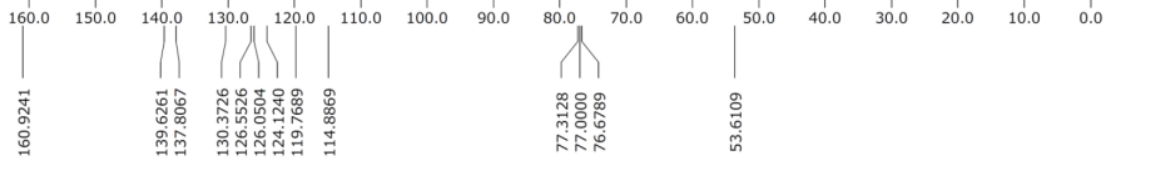



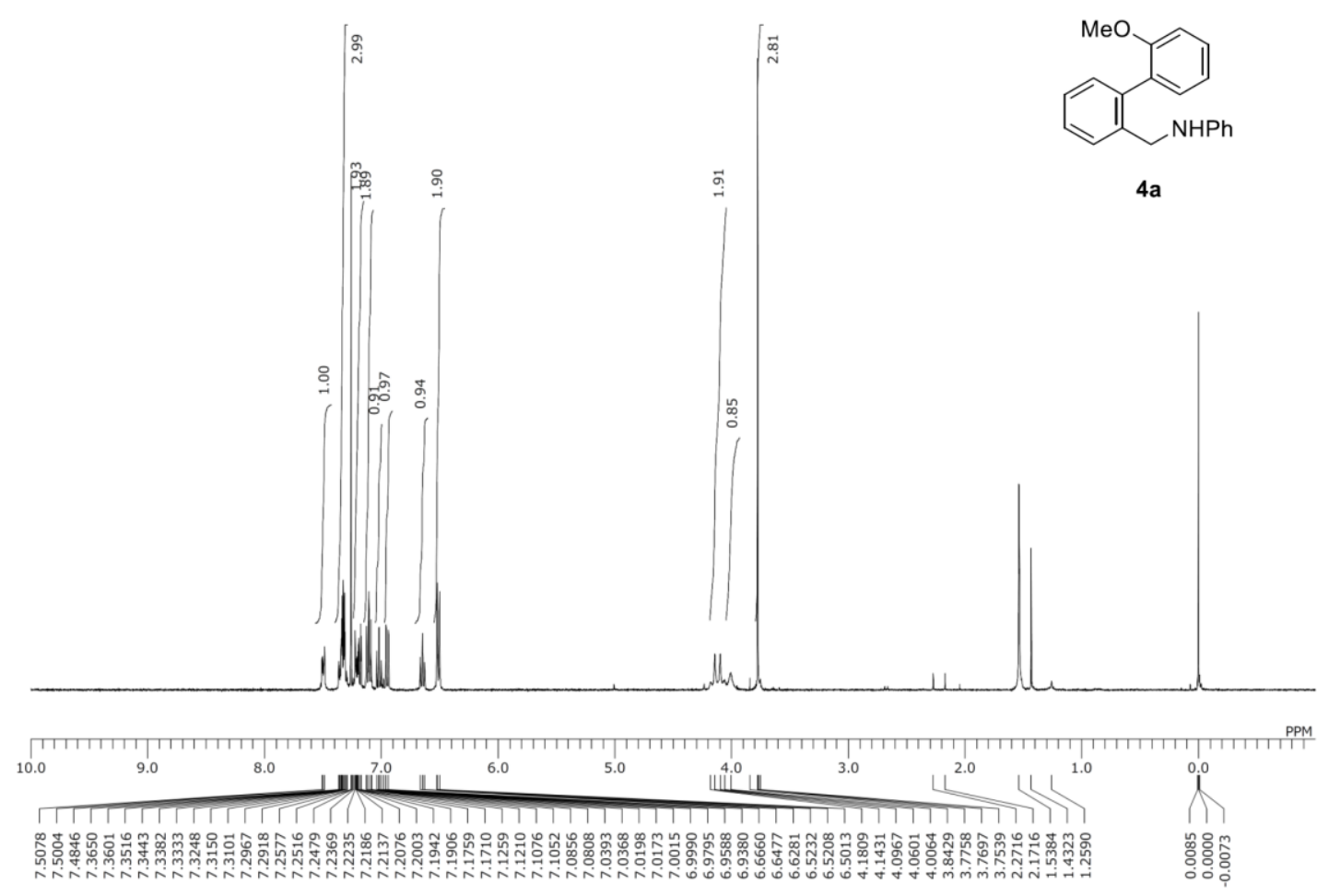

${ }^{13} \mathrm{C}$ NMR spectra of $\mathbf{4 a}$
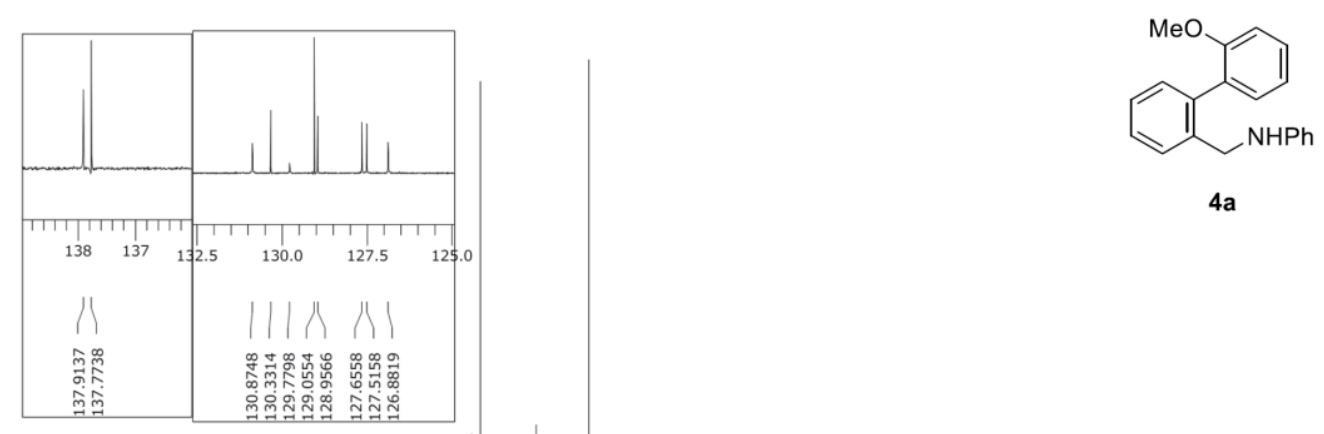

$4 a$

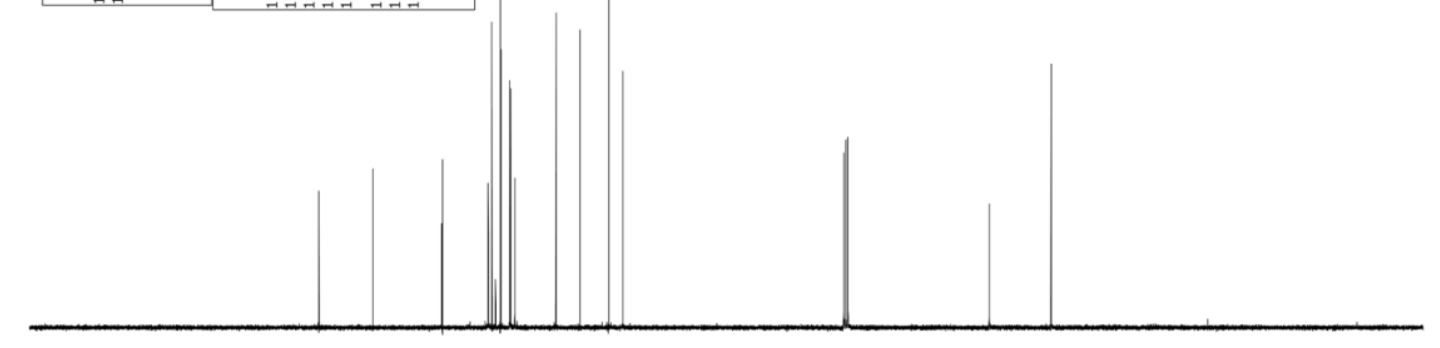

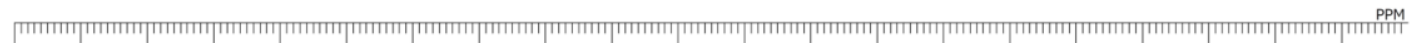

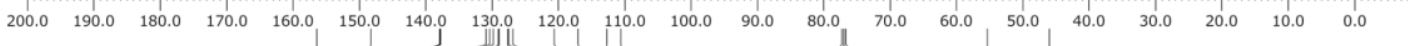

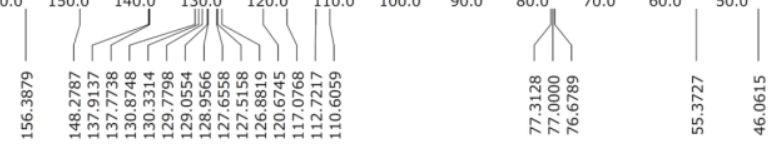




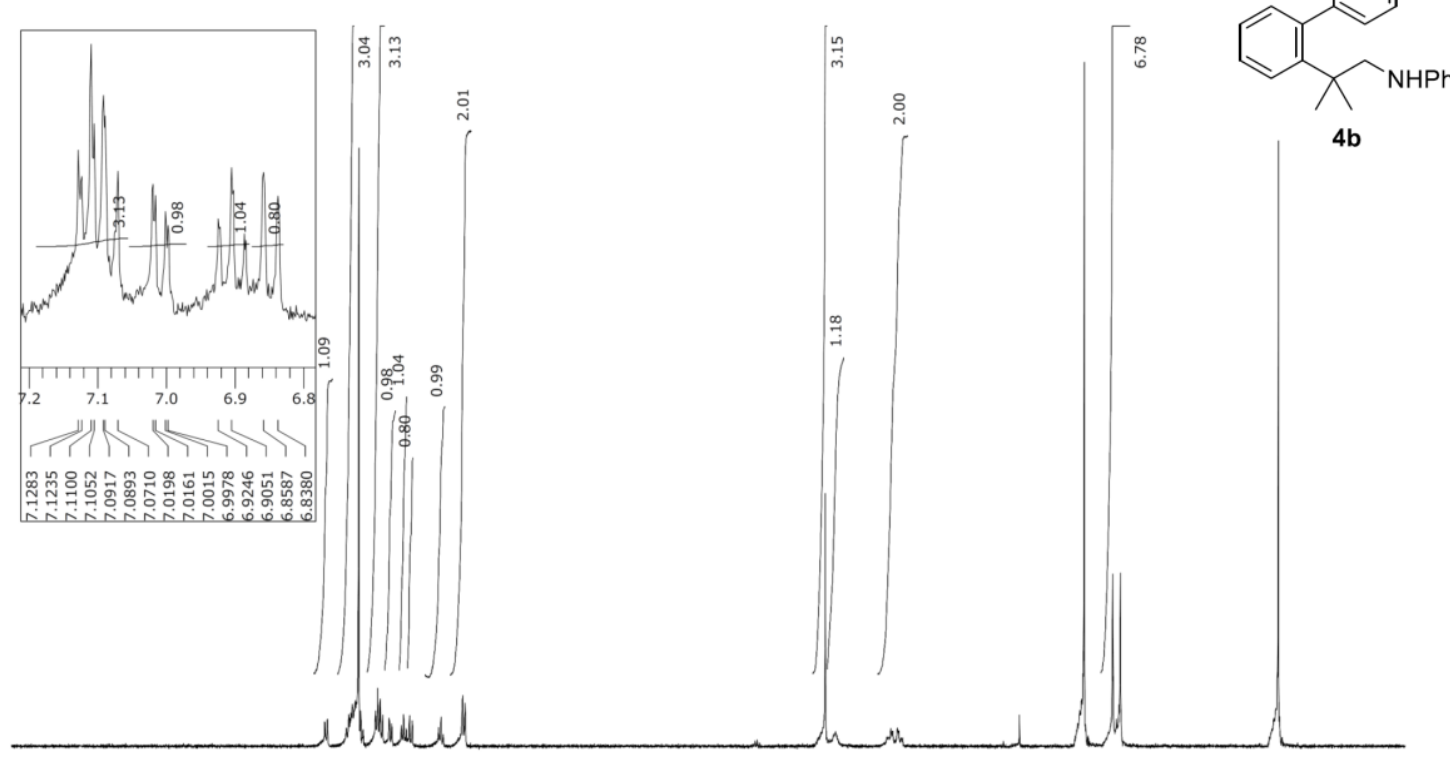

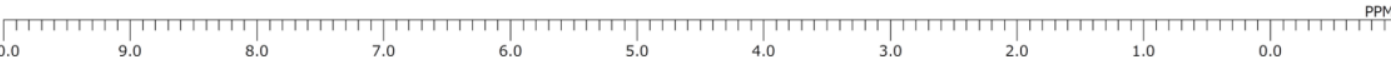

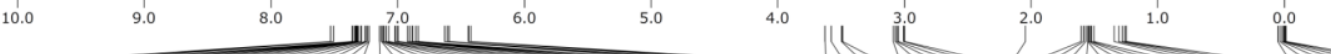

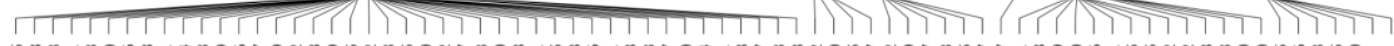

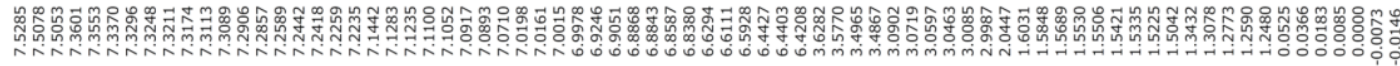

${ }^{13} \mathrm{C}$ NMR spectra of $\mathbf{4 b}$

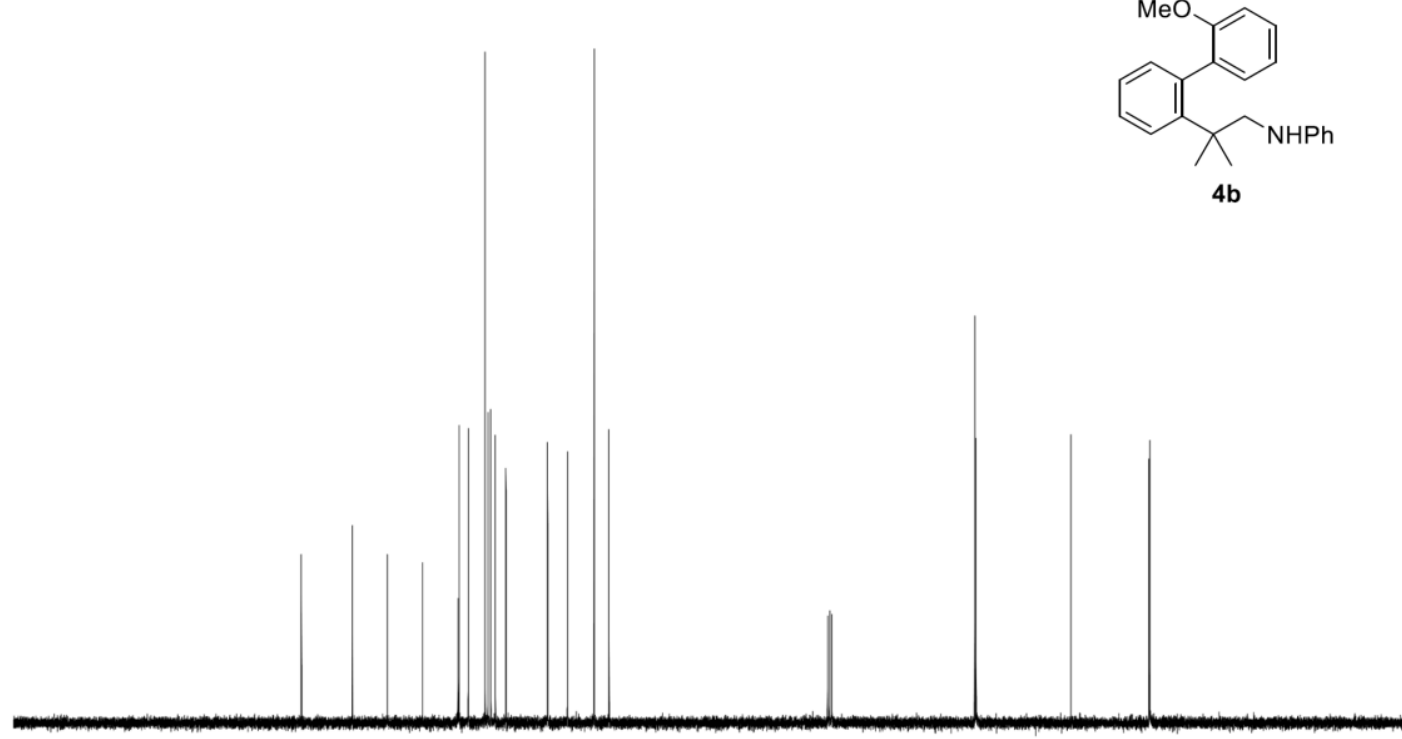

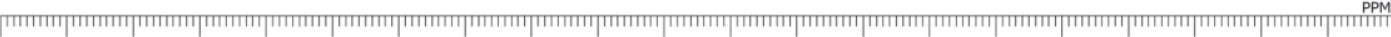
$\begin{array}{lllllllllllllllllllll}200.0 & 190.0 & 180.0 & 170.0 & 160.0 & 150.0 & 140.0 & 130.0 & 120.0 & 110.0 & 100.0 & 90.0 & 80.0 & 70.0 & 60.0 & 50.0 & 40.0 & 30.0 & 20.0 & 10.0 & 0.0\end{array}$

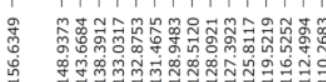

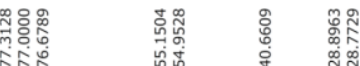



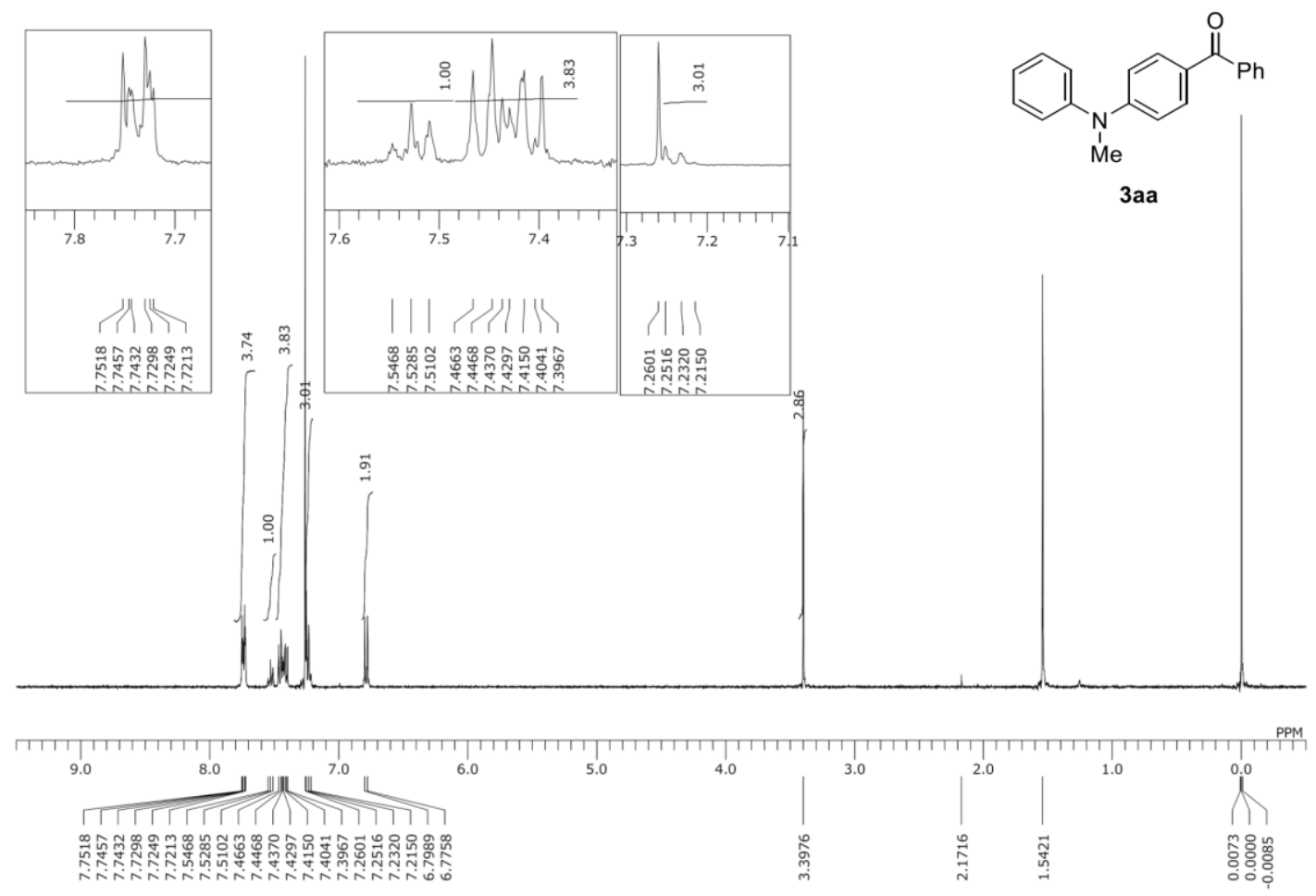

${ }^{13} \mathrm{C}$ NMR spectra of $\mathbf{3 a a}$

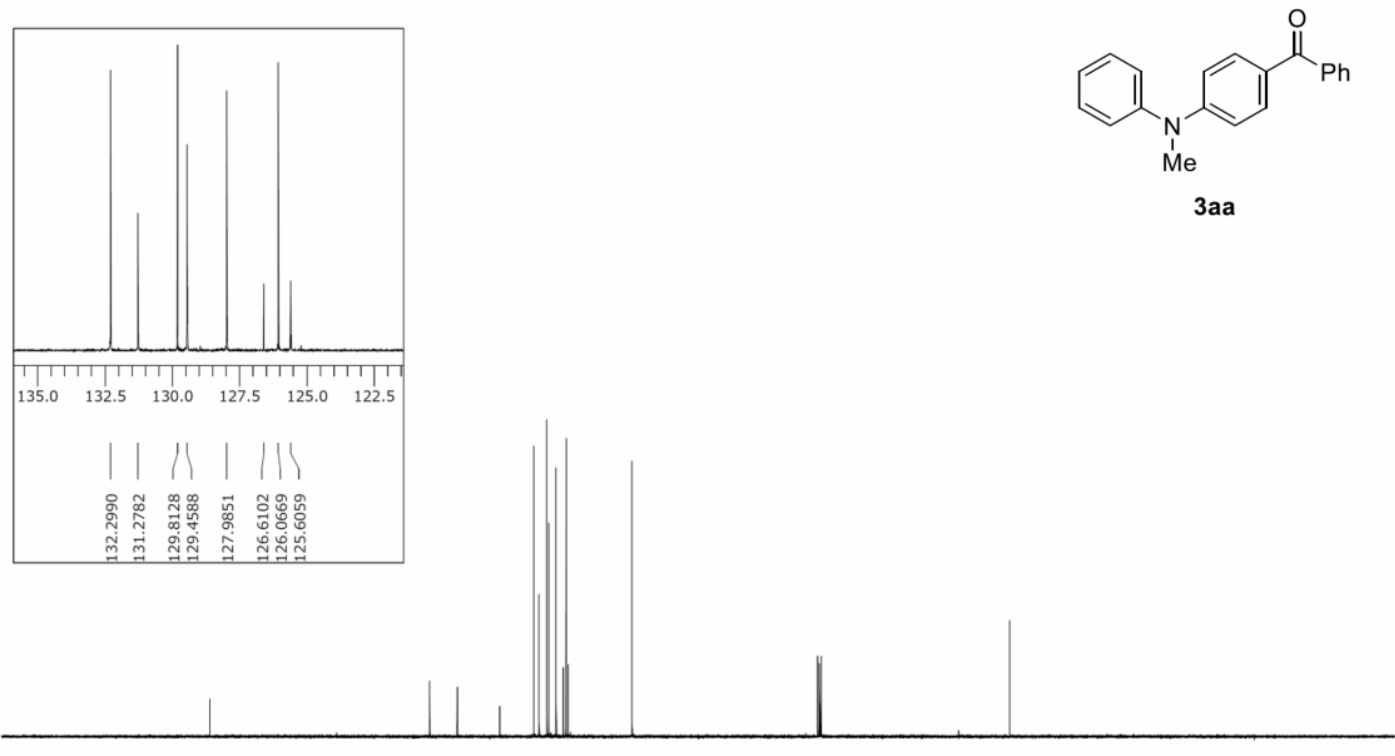

PPM

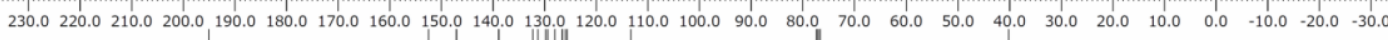

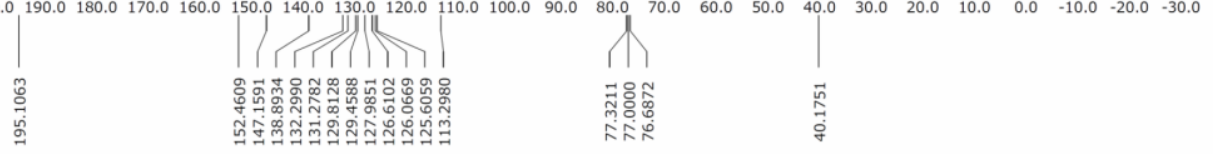


${ }^{1} \mathrm{H}$ NMR spectra of $\mathbf{3 a b}$

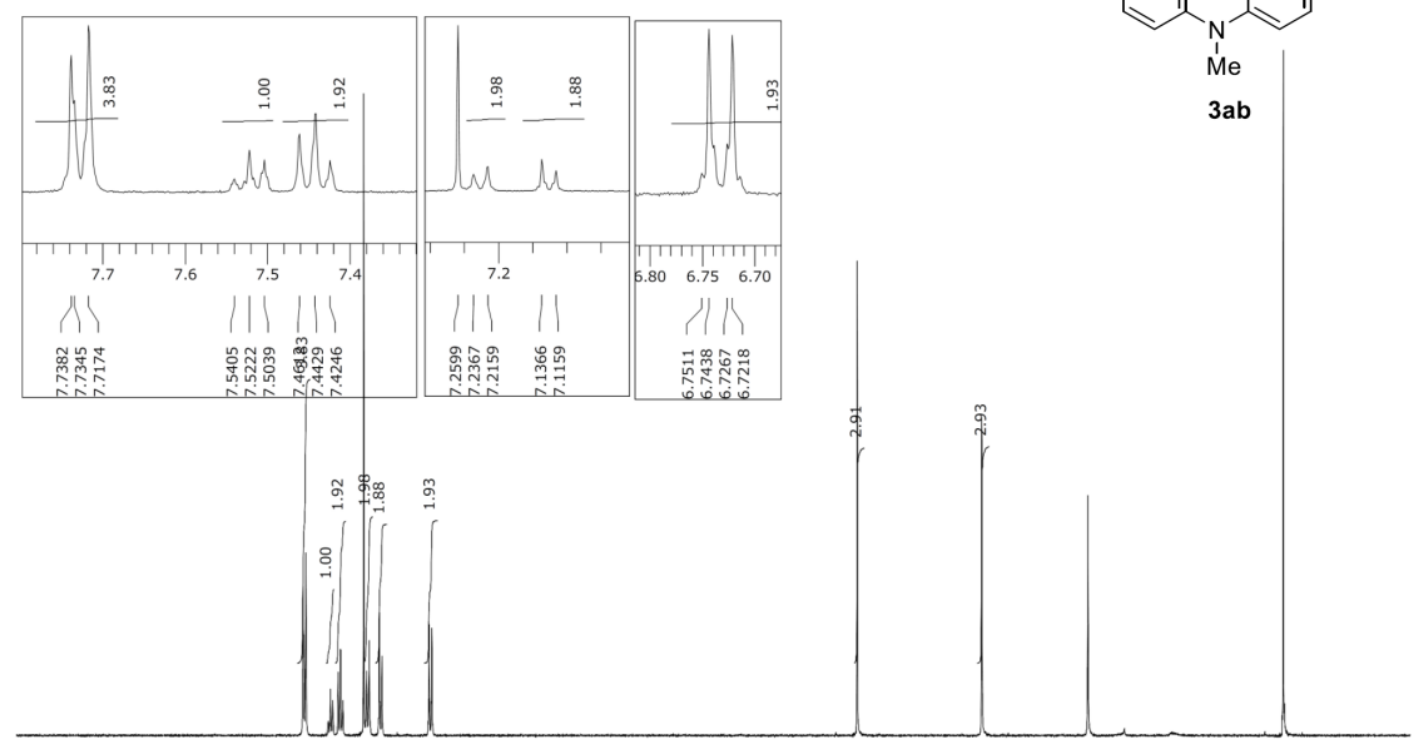

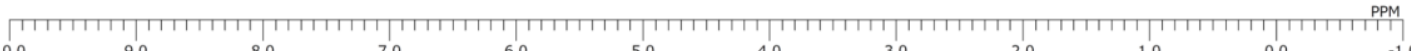

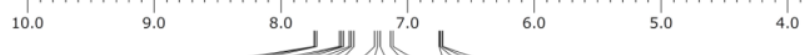
TाTा/TM

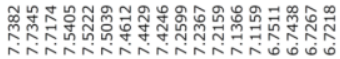

${ }^{13} \mathrm{C}$ NMR spectra of $\mathbf{3 a b}$
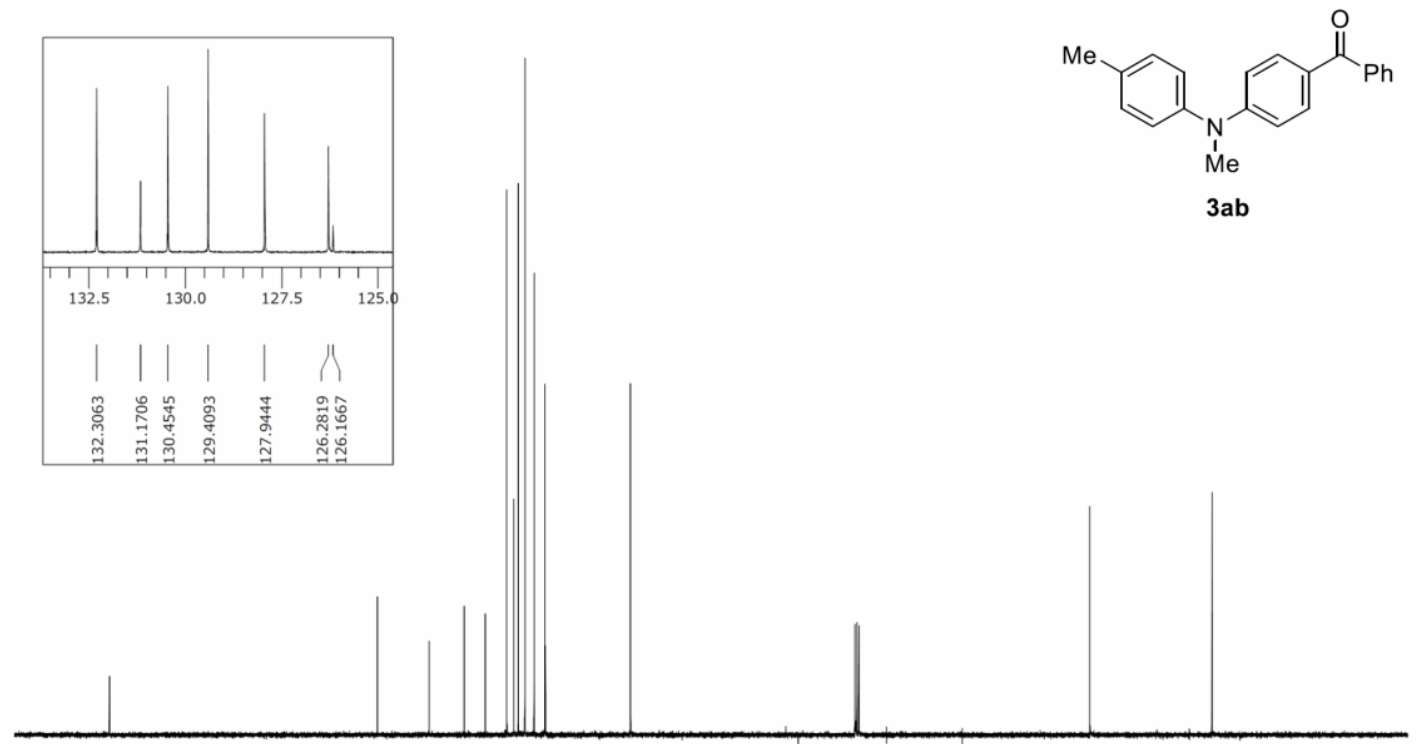

PPM

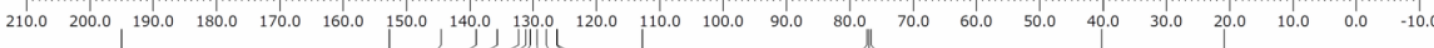

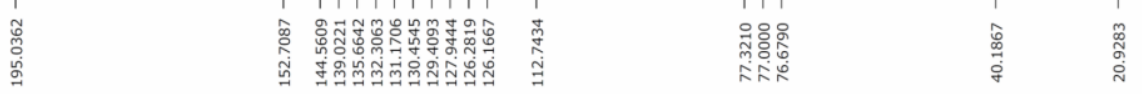




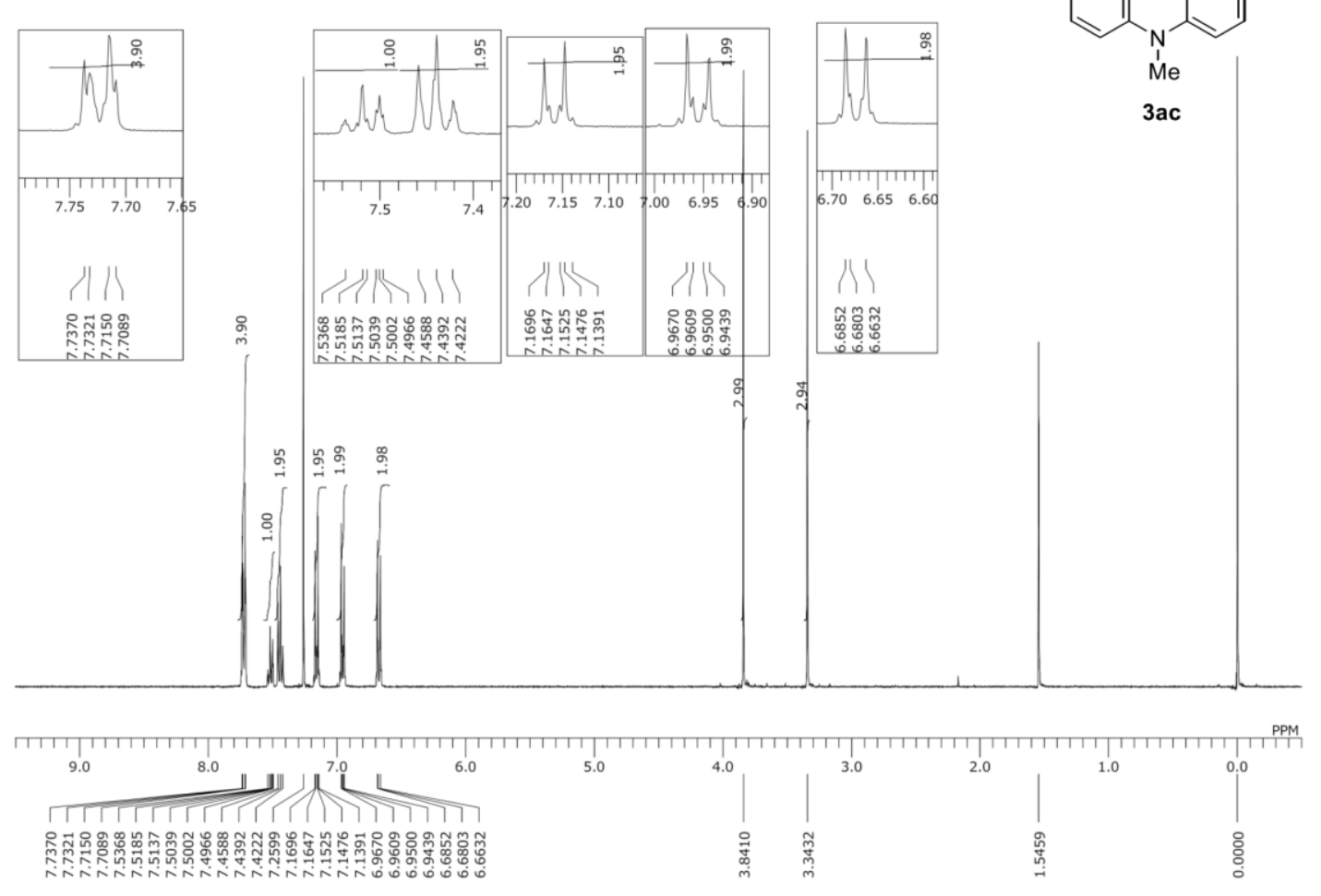

${ }^{13} \mathrm{C}$ NMR spectra of $\mathbf{3 a c}$
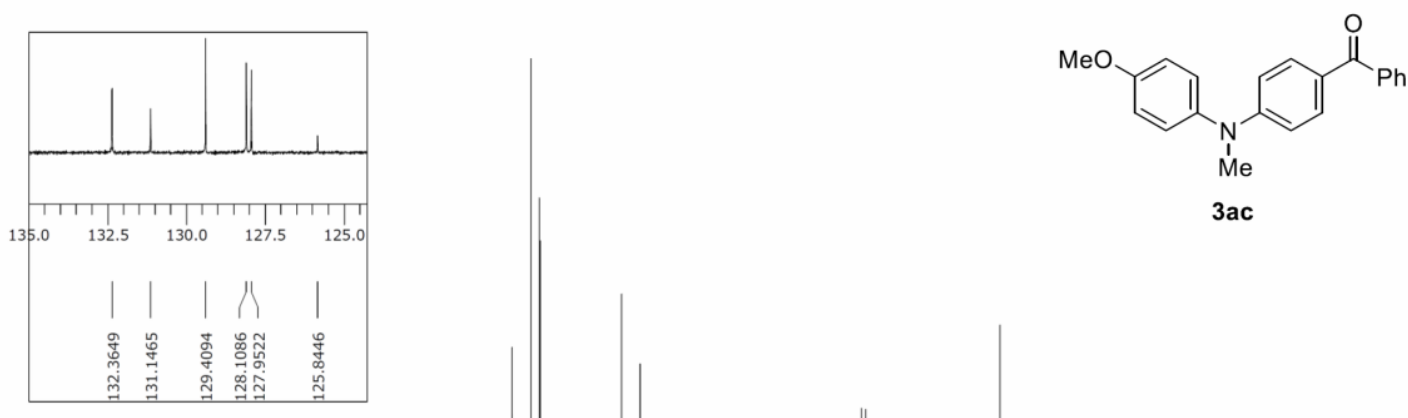

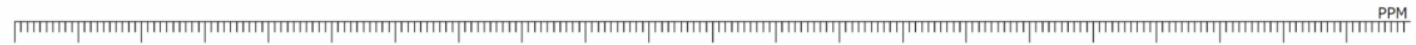

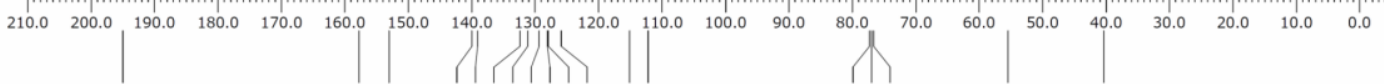

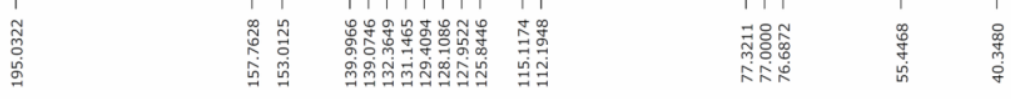



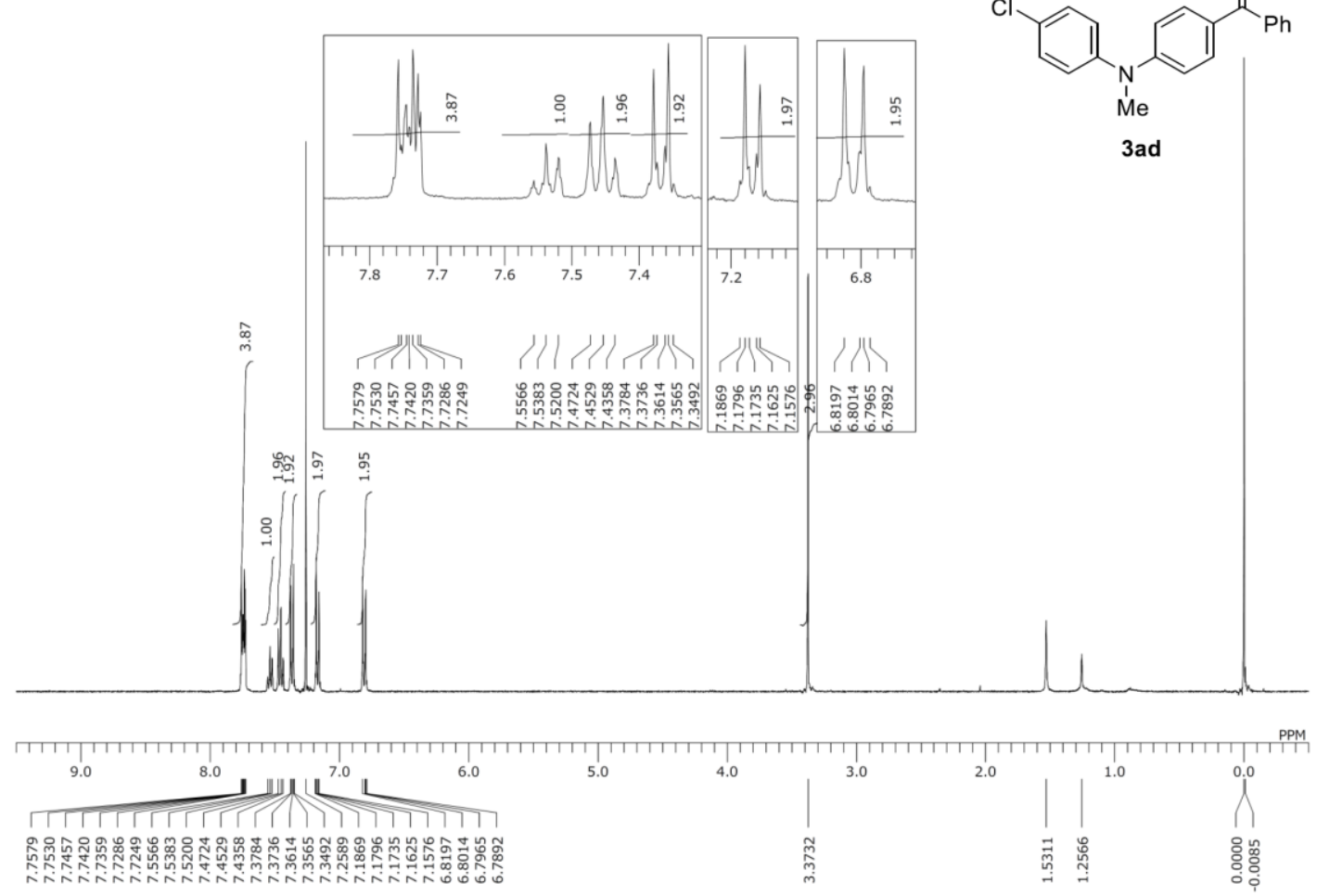

${ }^{13} \mathrm{C}$ NMR spectra of $\mathbf{3 a d}$
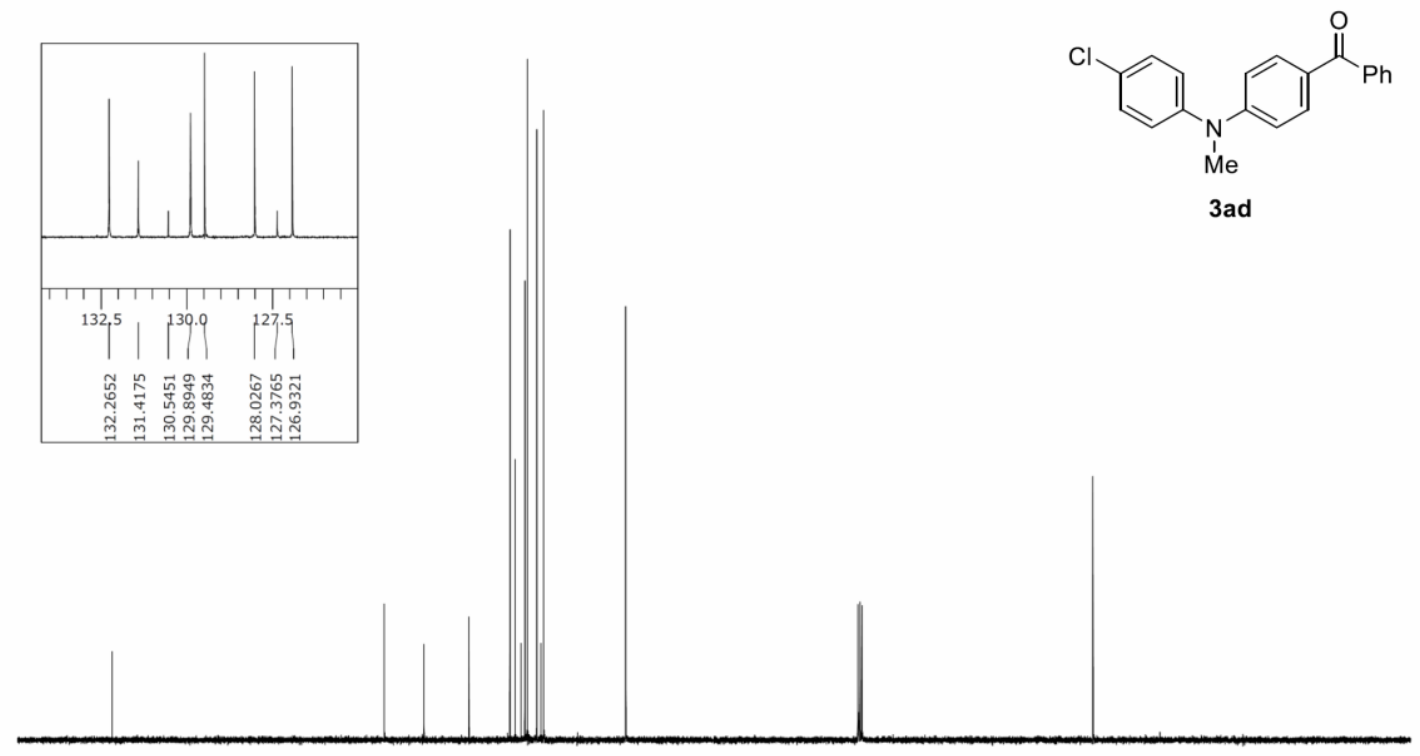

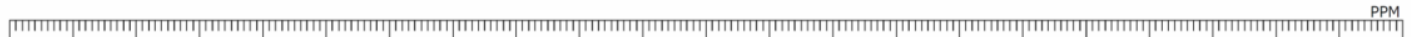

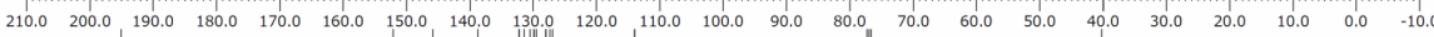

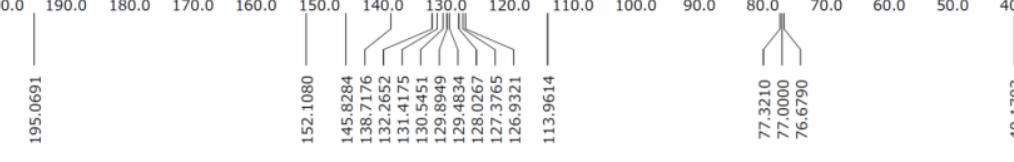



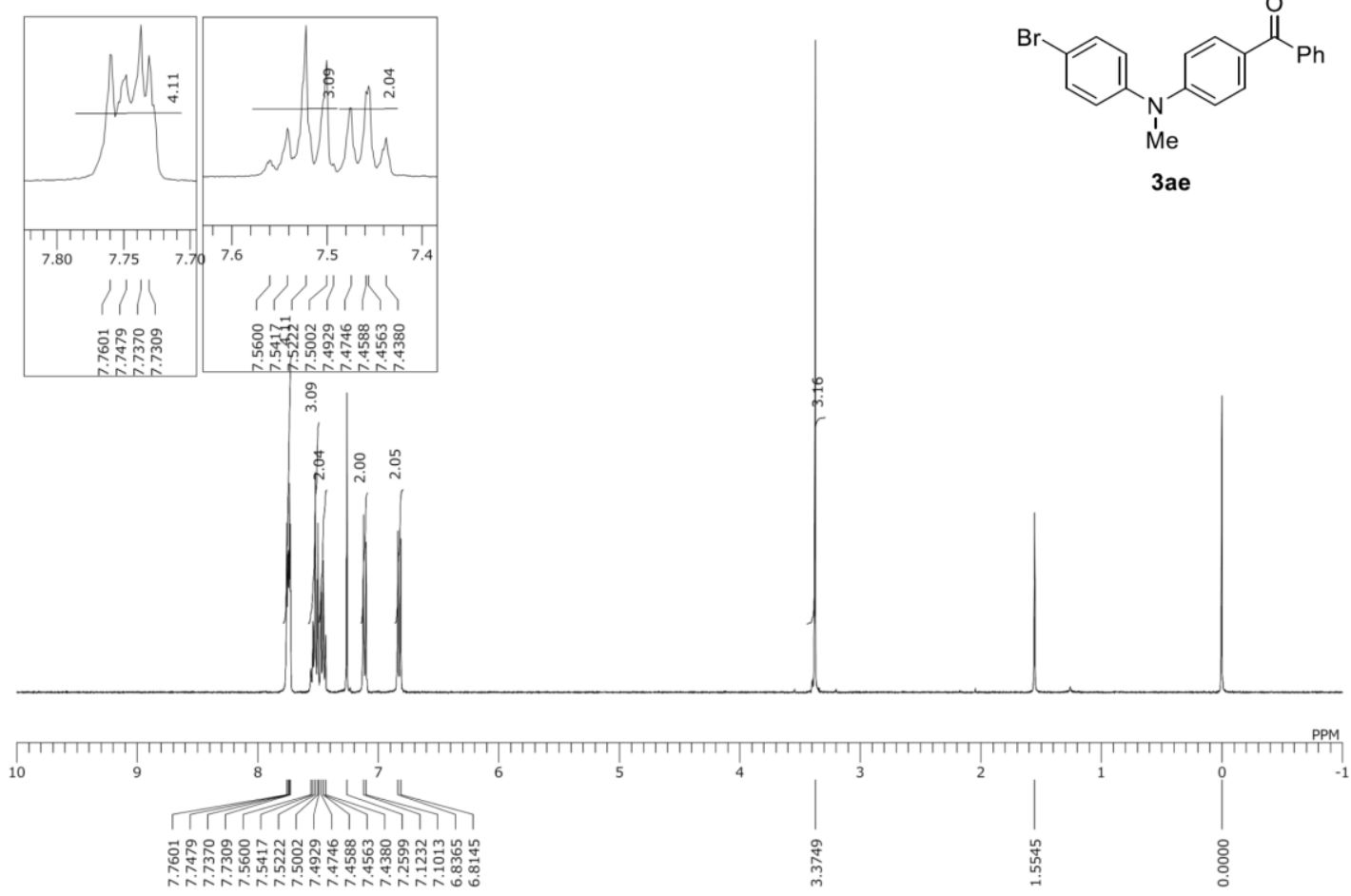

${ }^{13} \mathrm{C}$ NMR spectra of $\mathbf{3 a e}$

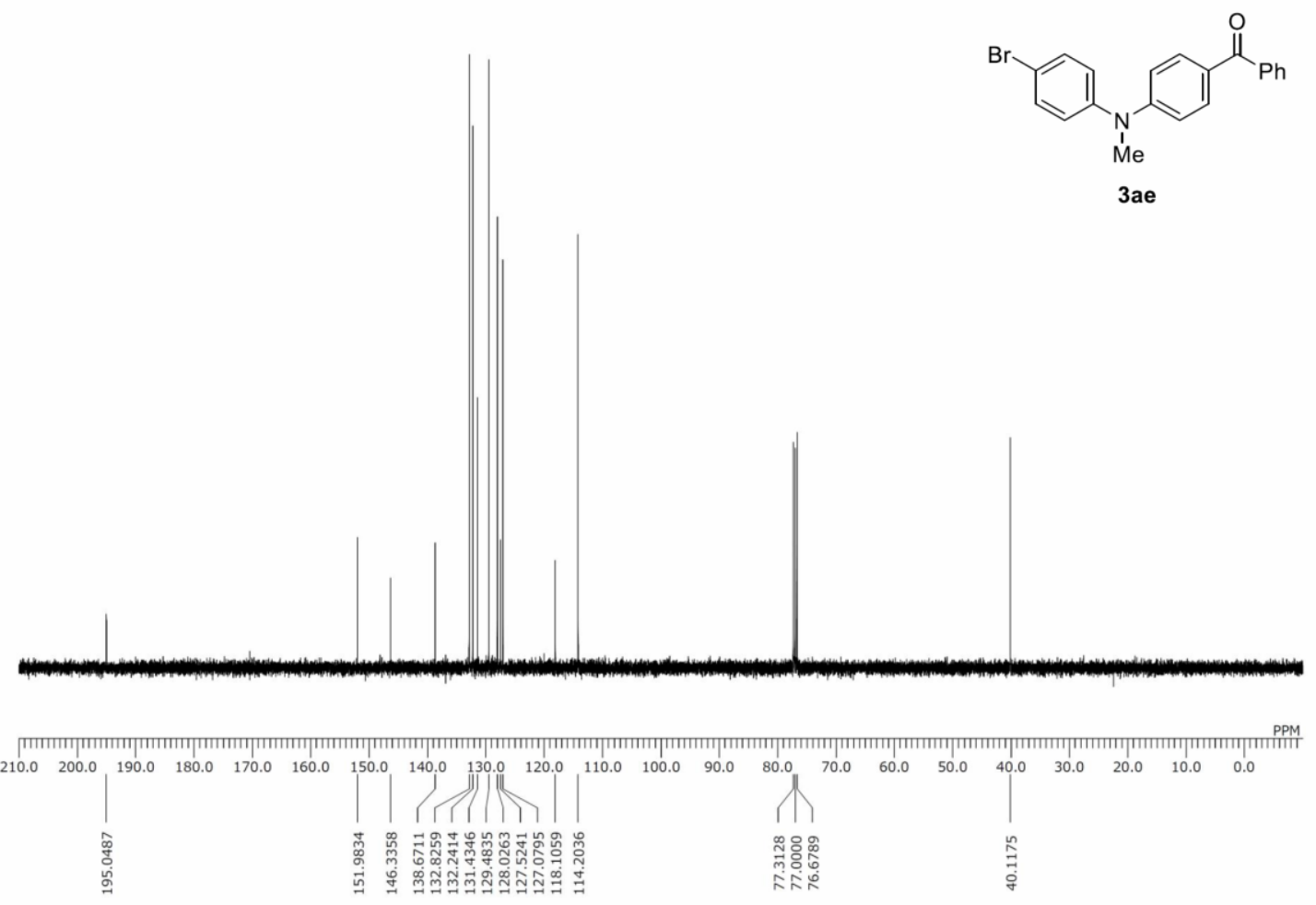



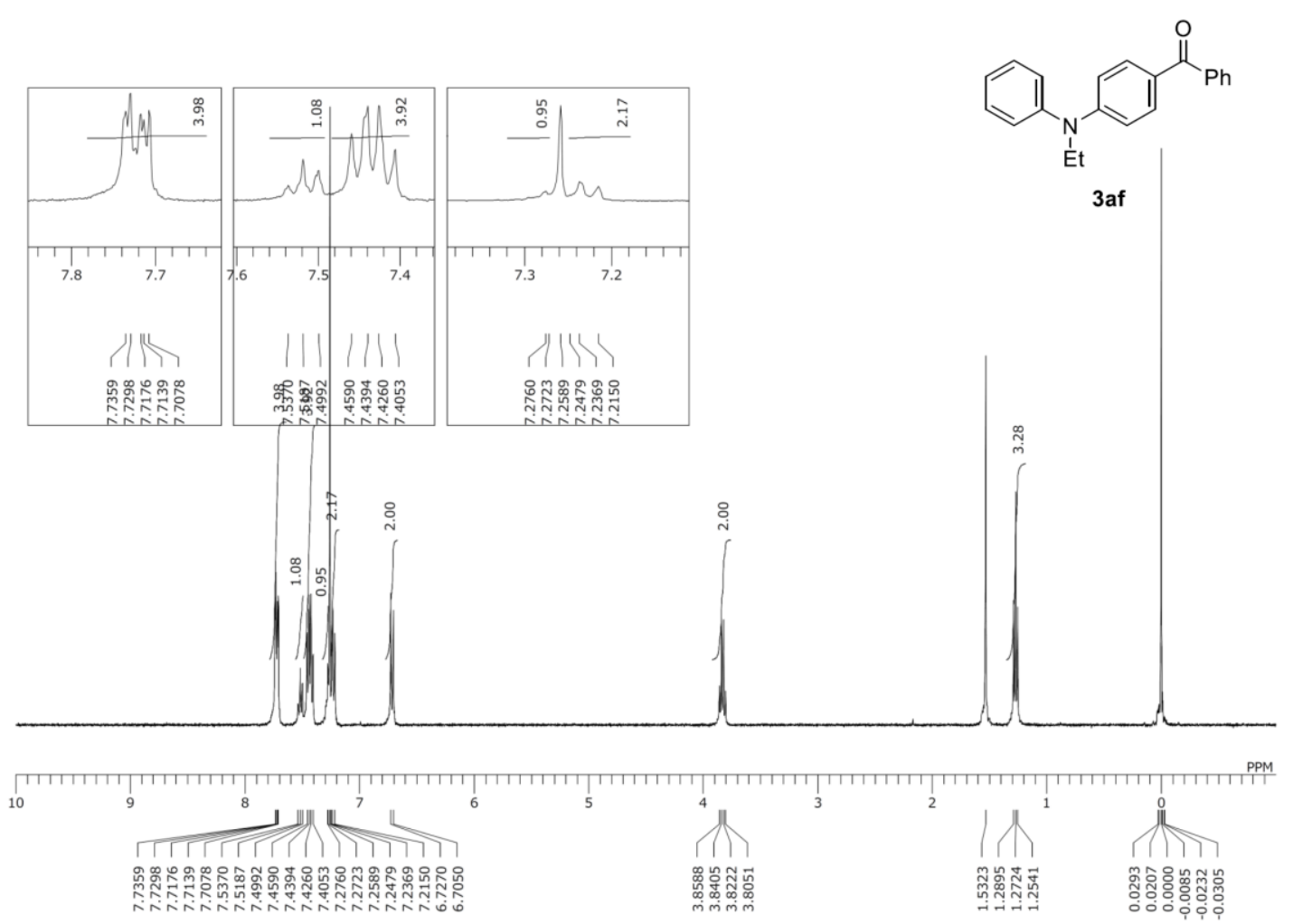

${ }^{13} \mathrm{C}$ NMR spectra of $\mathbf{3 a f}$
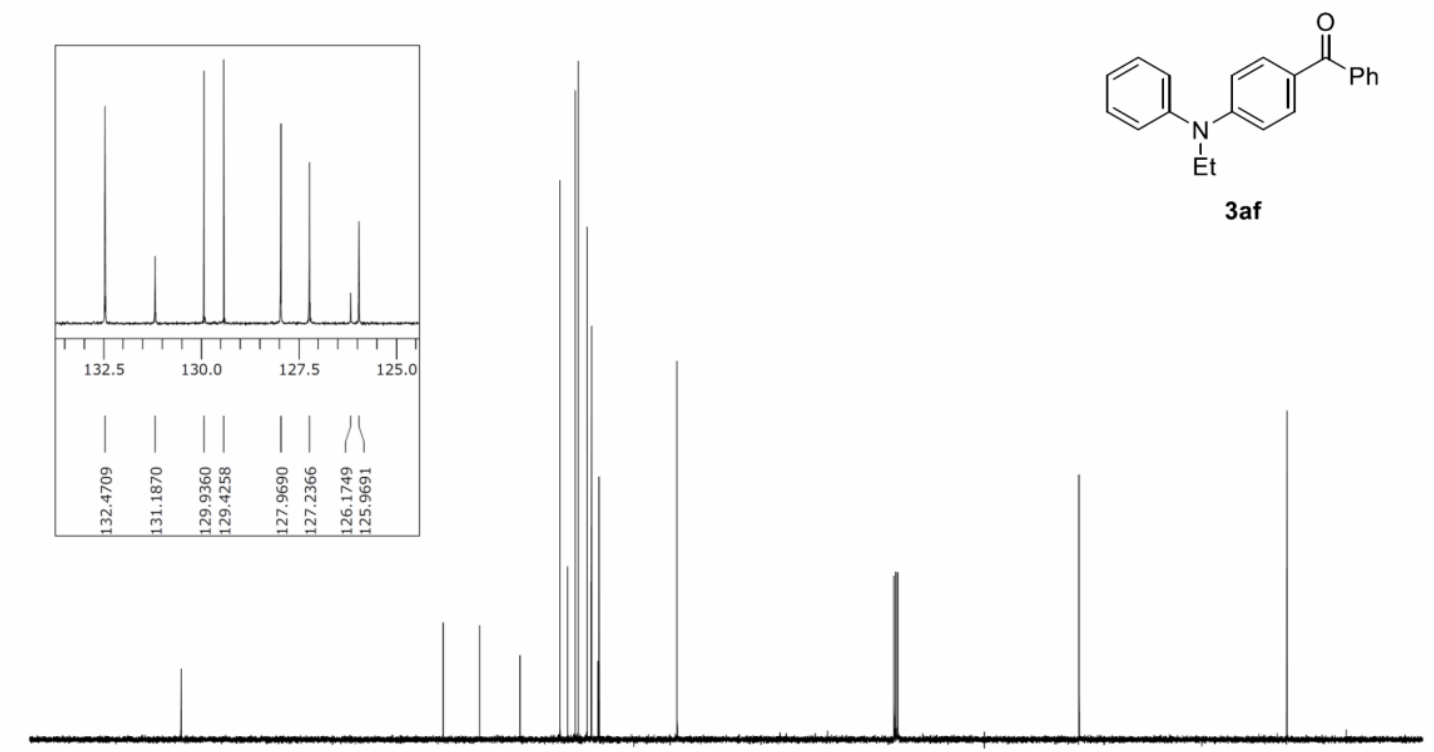

PPM

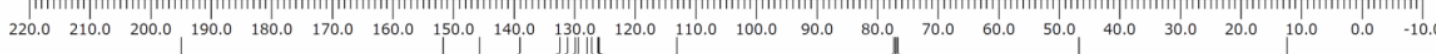

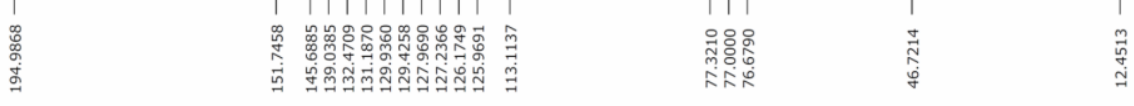



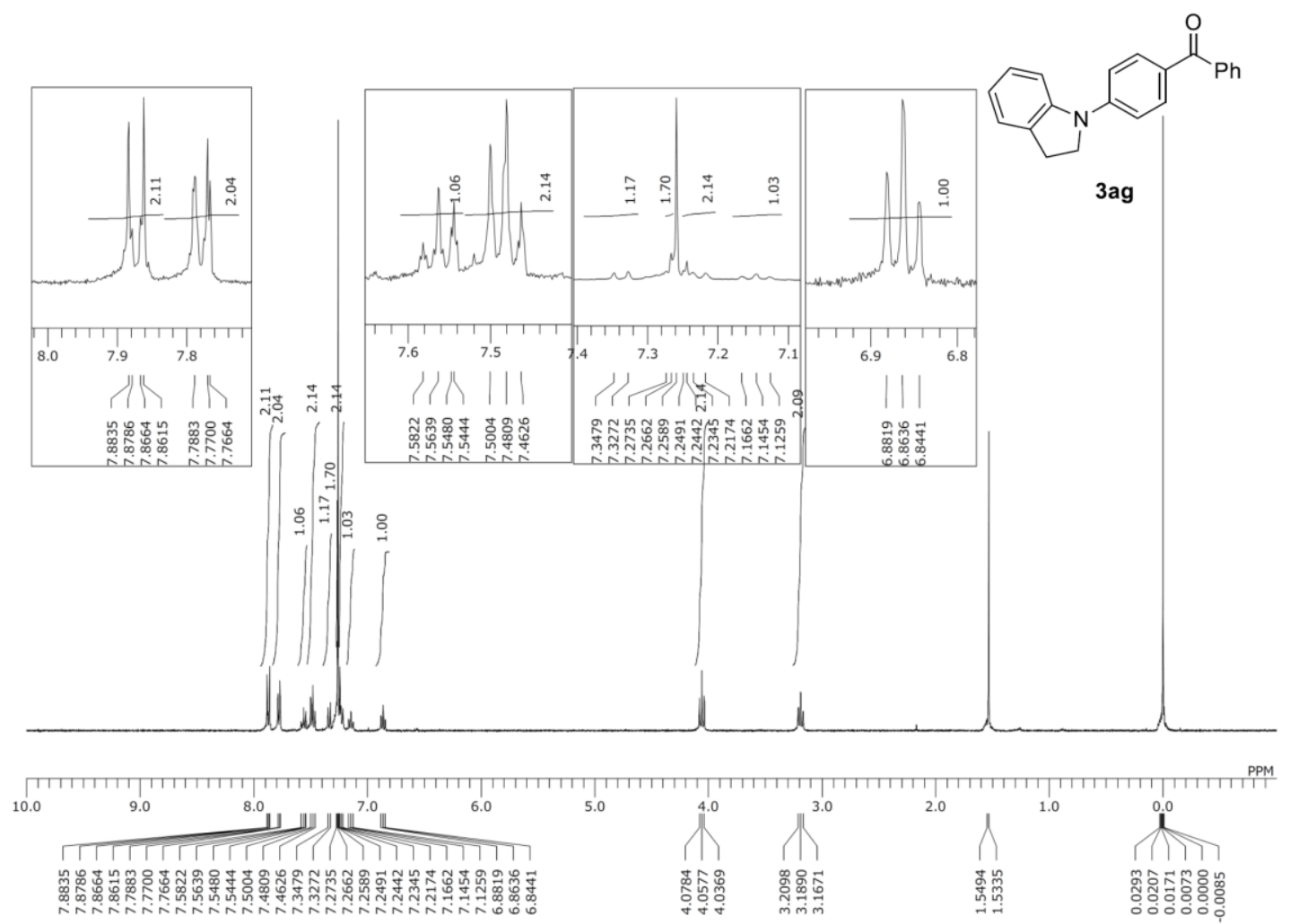

${ }^{13} \mathrm{C}$ NMR spectra of $\mathbf{3 a g}$
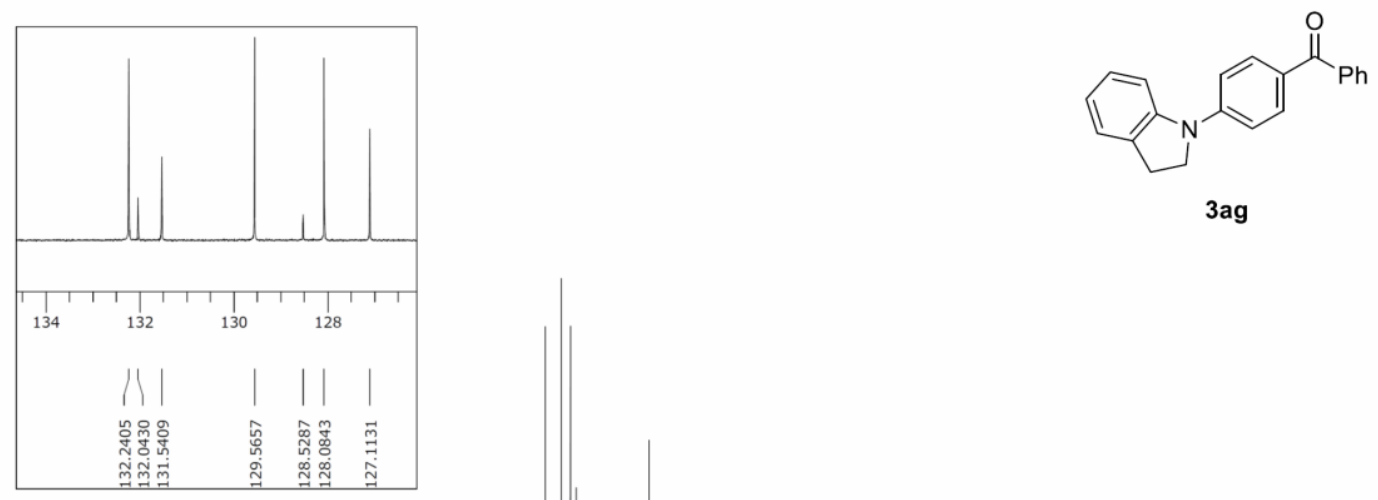

3ag

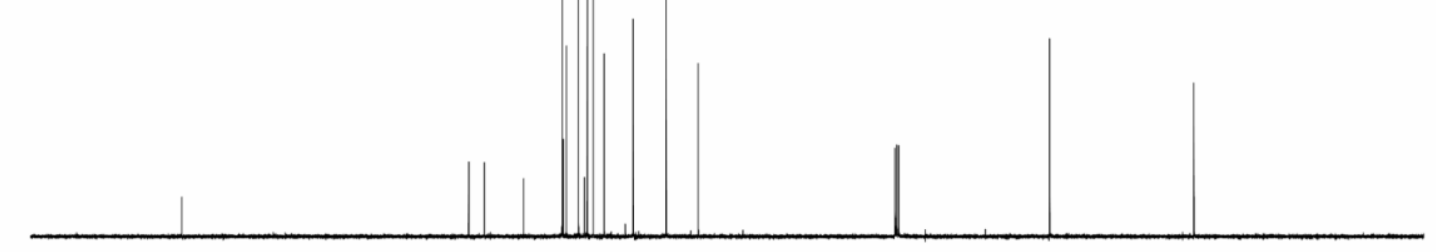

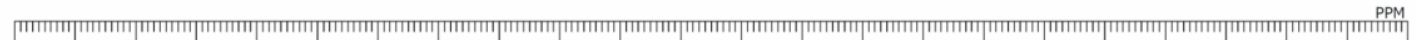

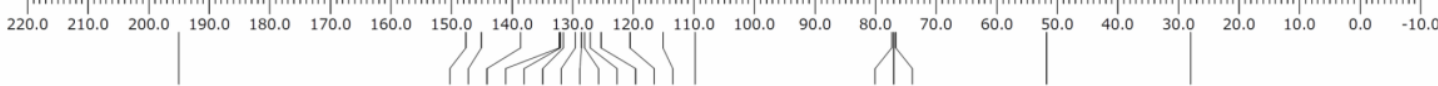

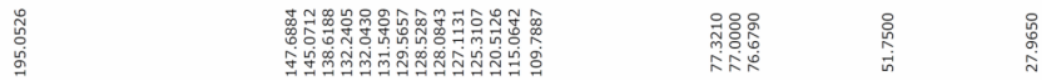




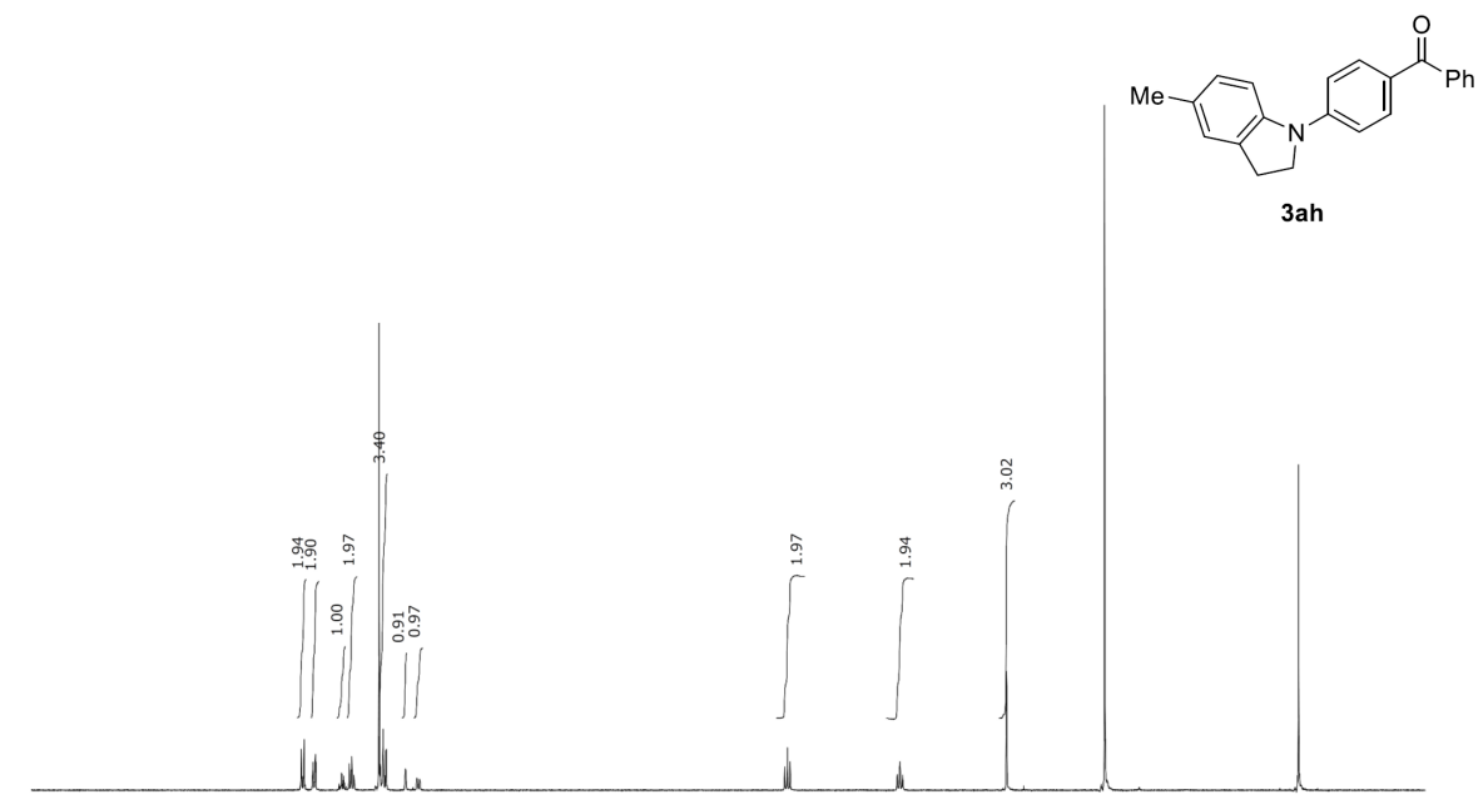

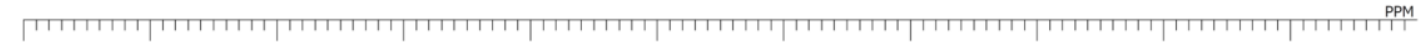
10.0

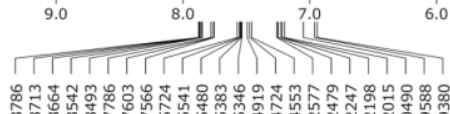

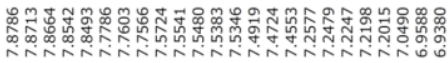

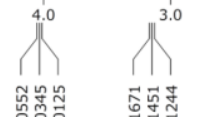

2.0

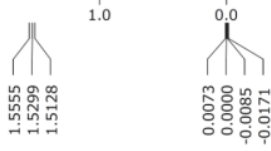

${ }^{13} \mathrm{C}$ NMR spectra of $\mathbf{3 a h}$
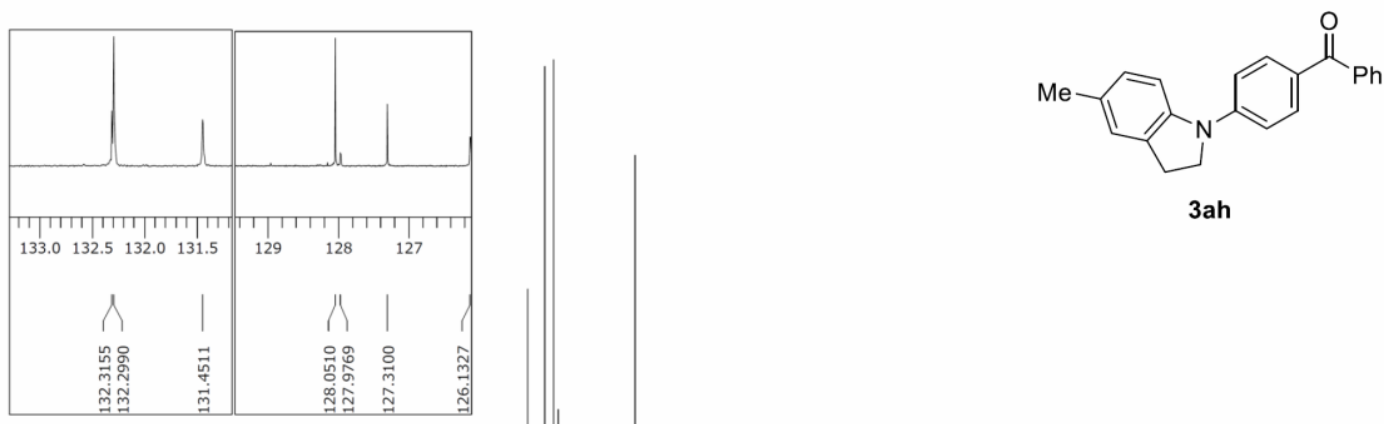

3ah

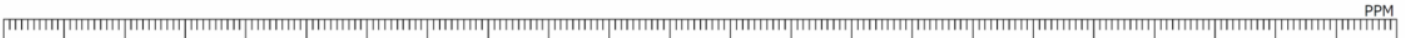

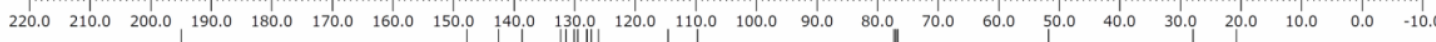

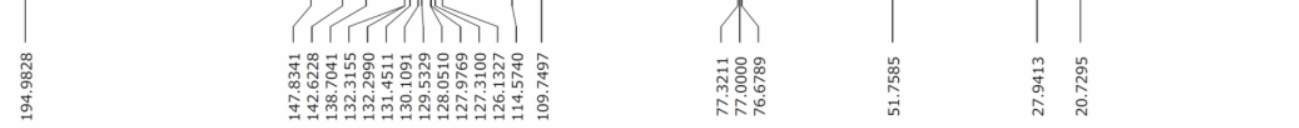



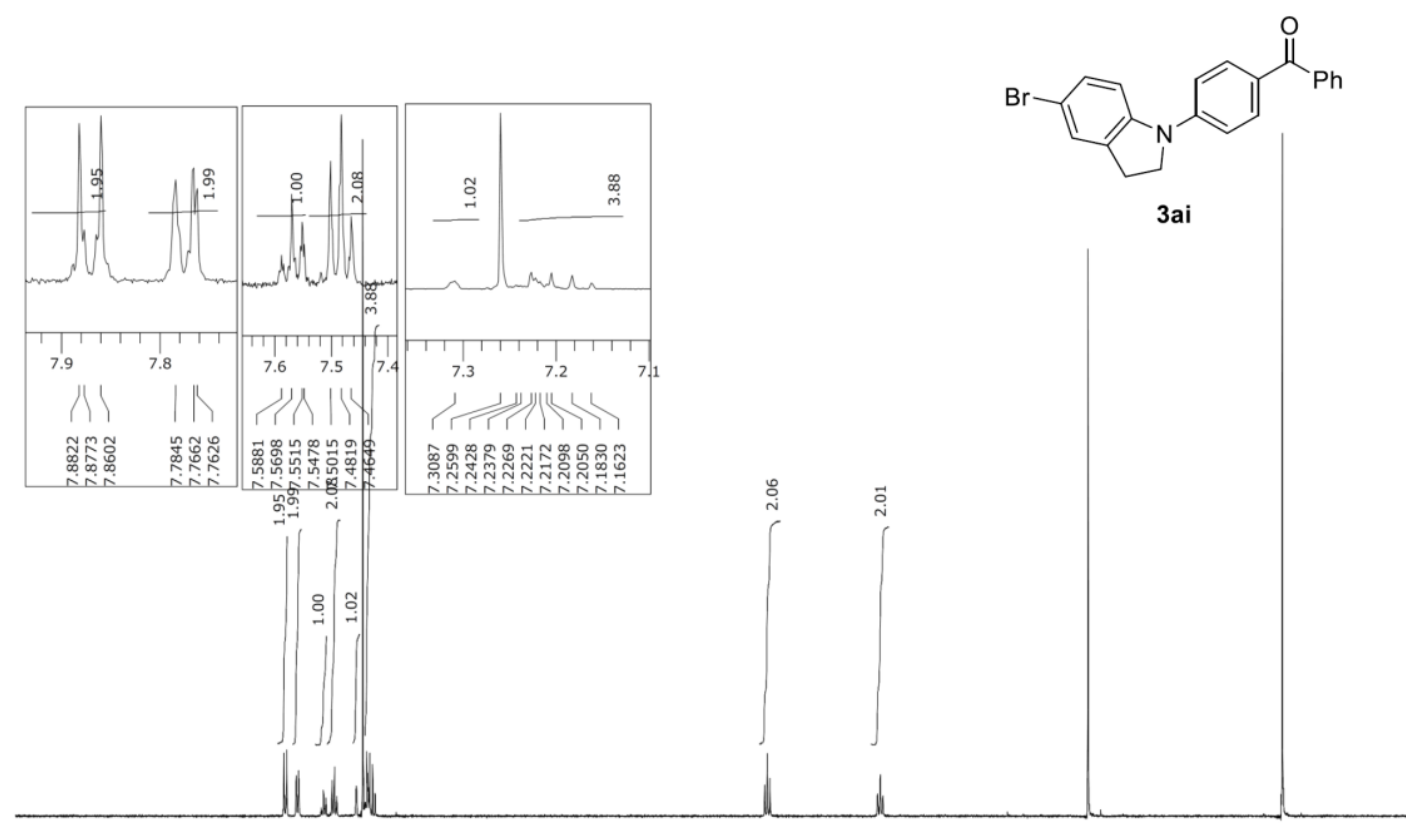

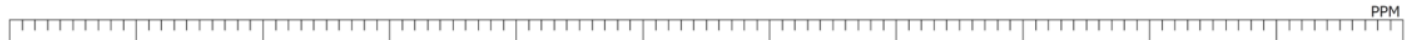
10.0

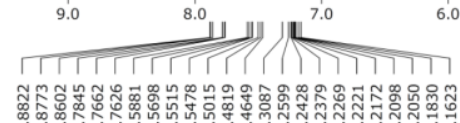

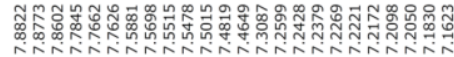

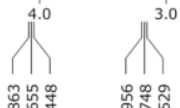

2.0

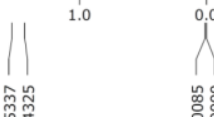

${ }^{13} \mathrm{C}$ NMR spectra of $\mathbf{3 a i}$

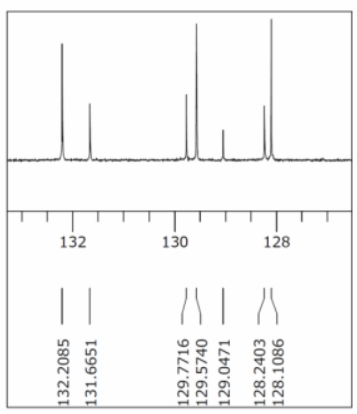

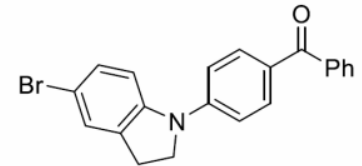

3ai

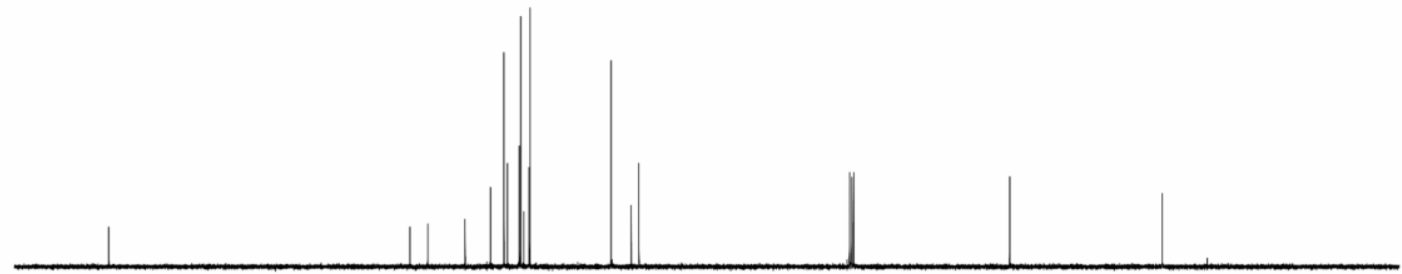

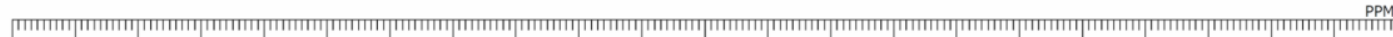
$\begin{array}{llllllllllllllllllllll}210.0 & 200.0 & 190.0 & 180.0 & 170.0 & 160.0 & 150.0 & 140.0 & 130.0 & 120.0 & 110.0 & 100.0 & 90.0 & 80.0 & 70.0 & 60.0 & 50.0 & 40.0 & 30.0 & 20.0 & 10.0 & 0.0\end{array}$

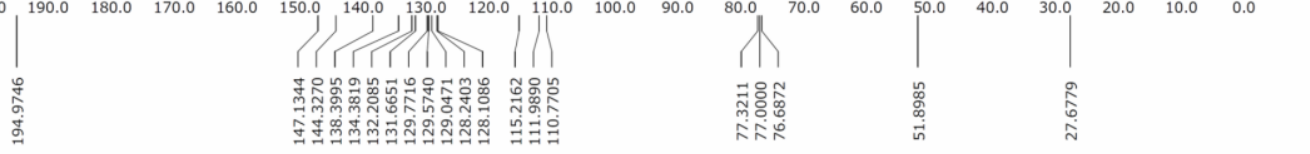



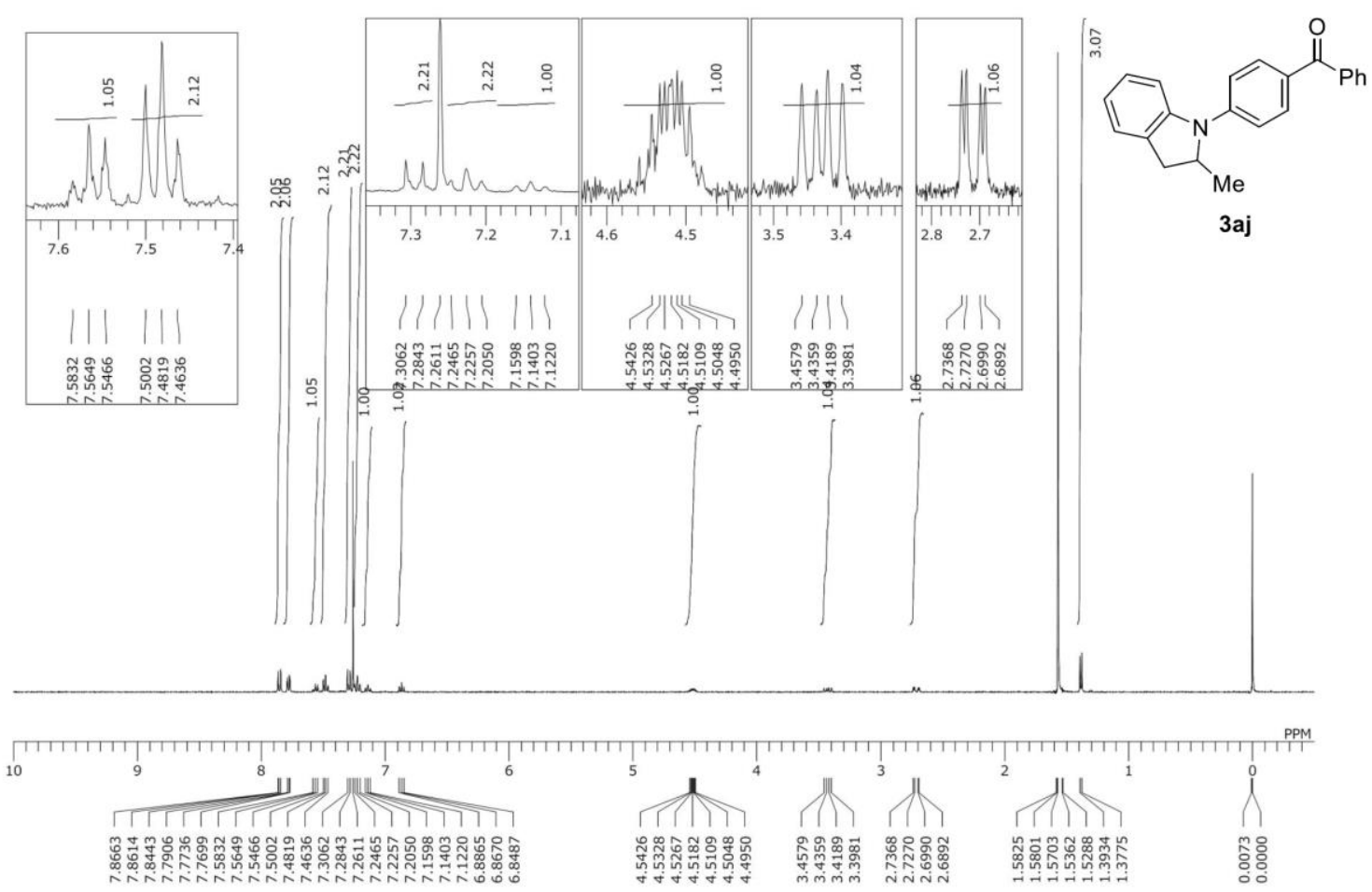

${ }^{13} \mathrm{C}$ NMR spectra of $\mathbf{3 a j}$

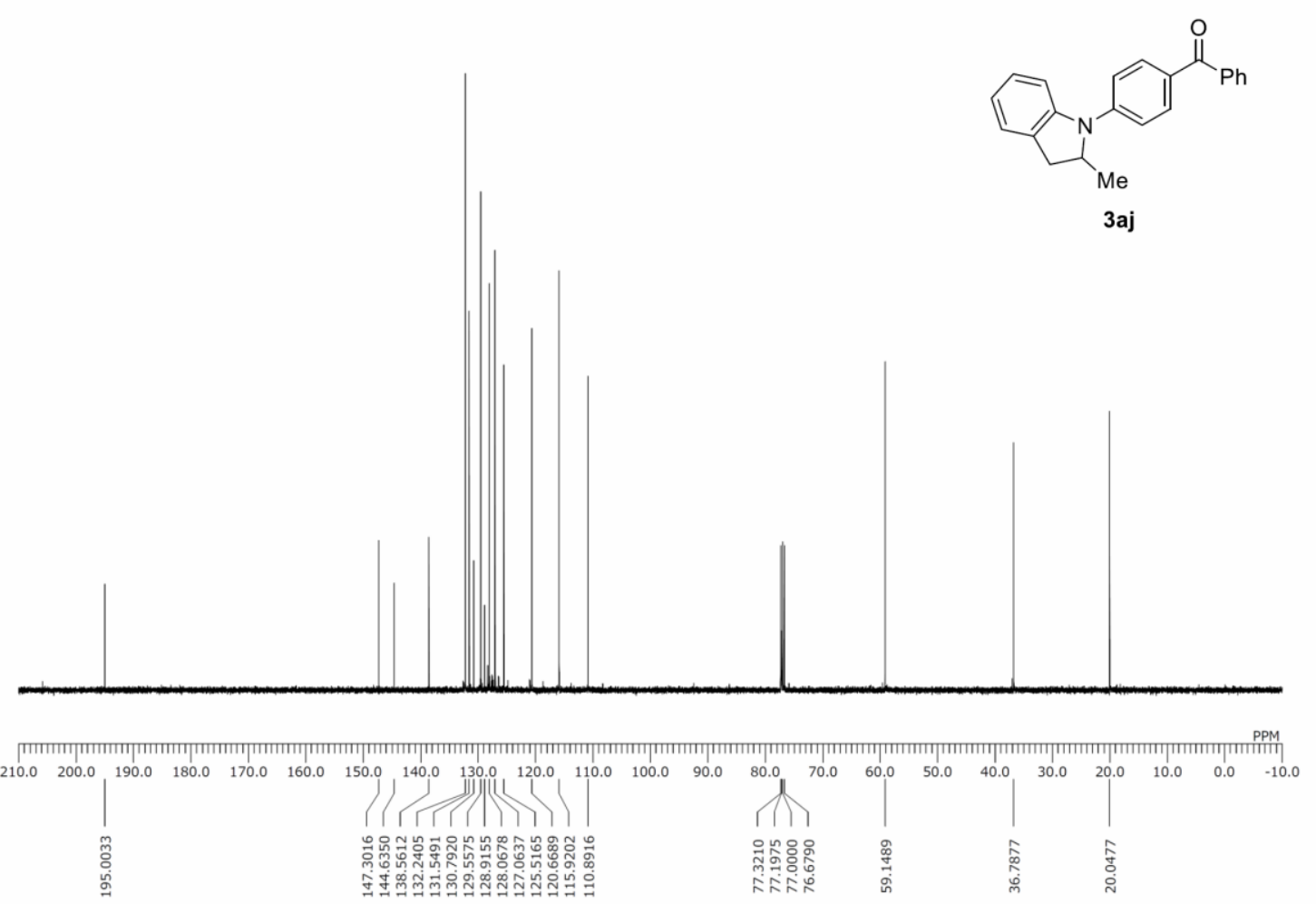




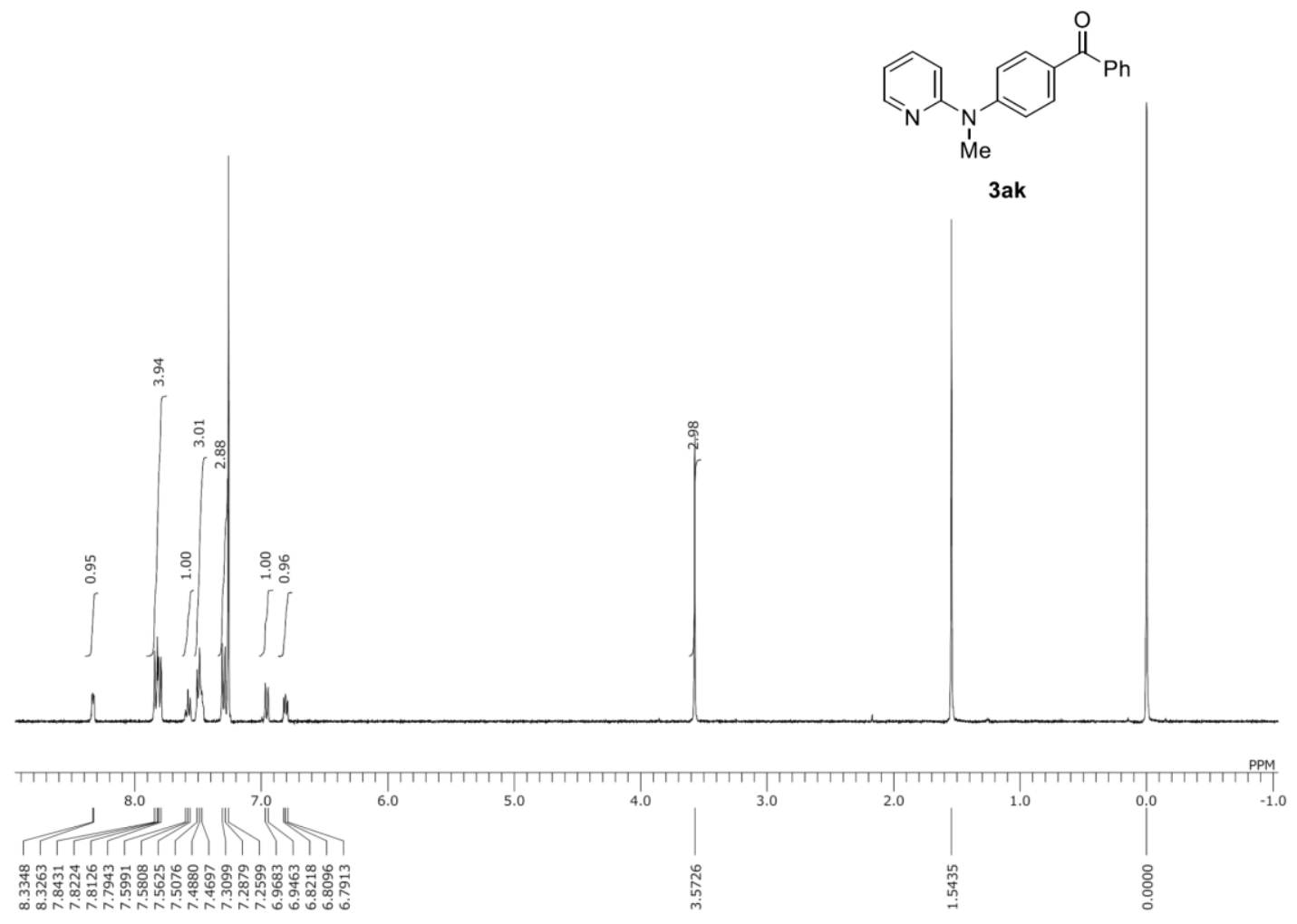

${ }^{13} \mathrm{C}$ NMR spectra of $\mathbf{3 a k}$
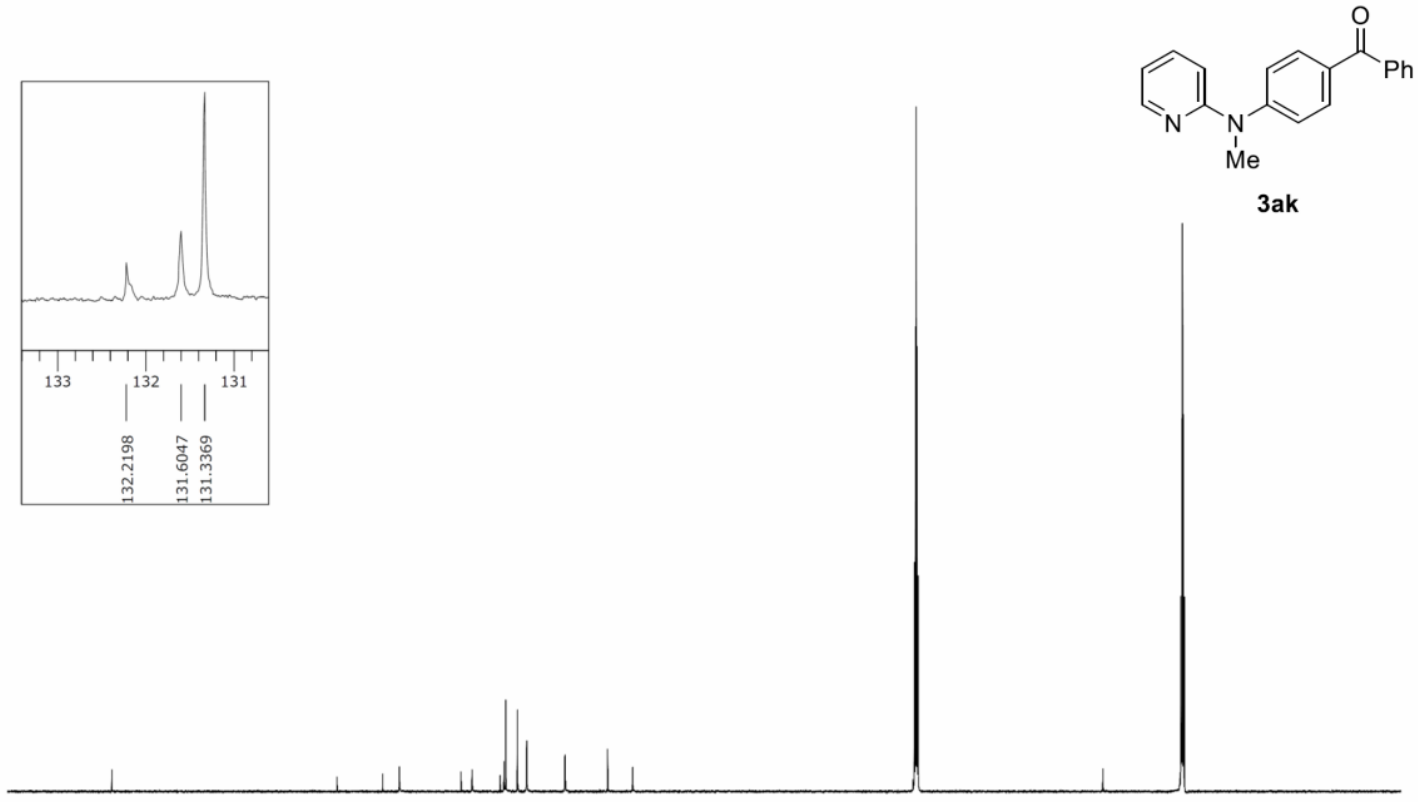

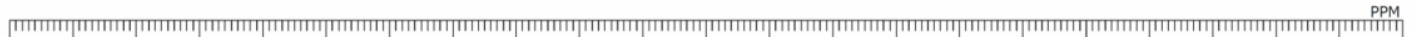

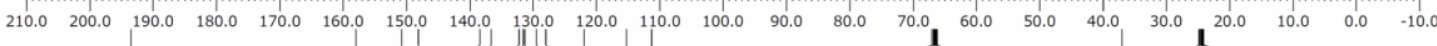

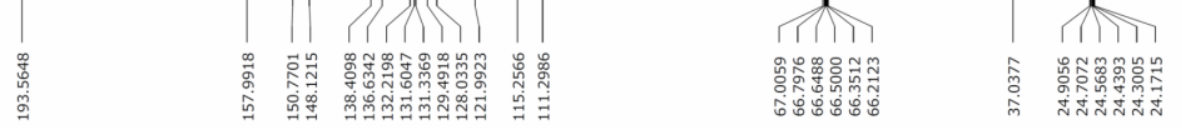



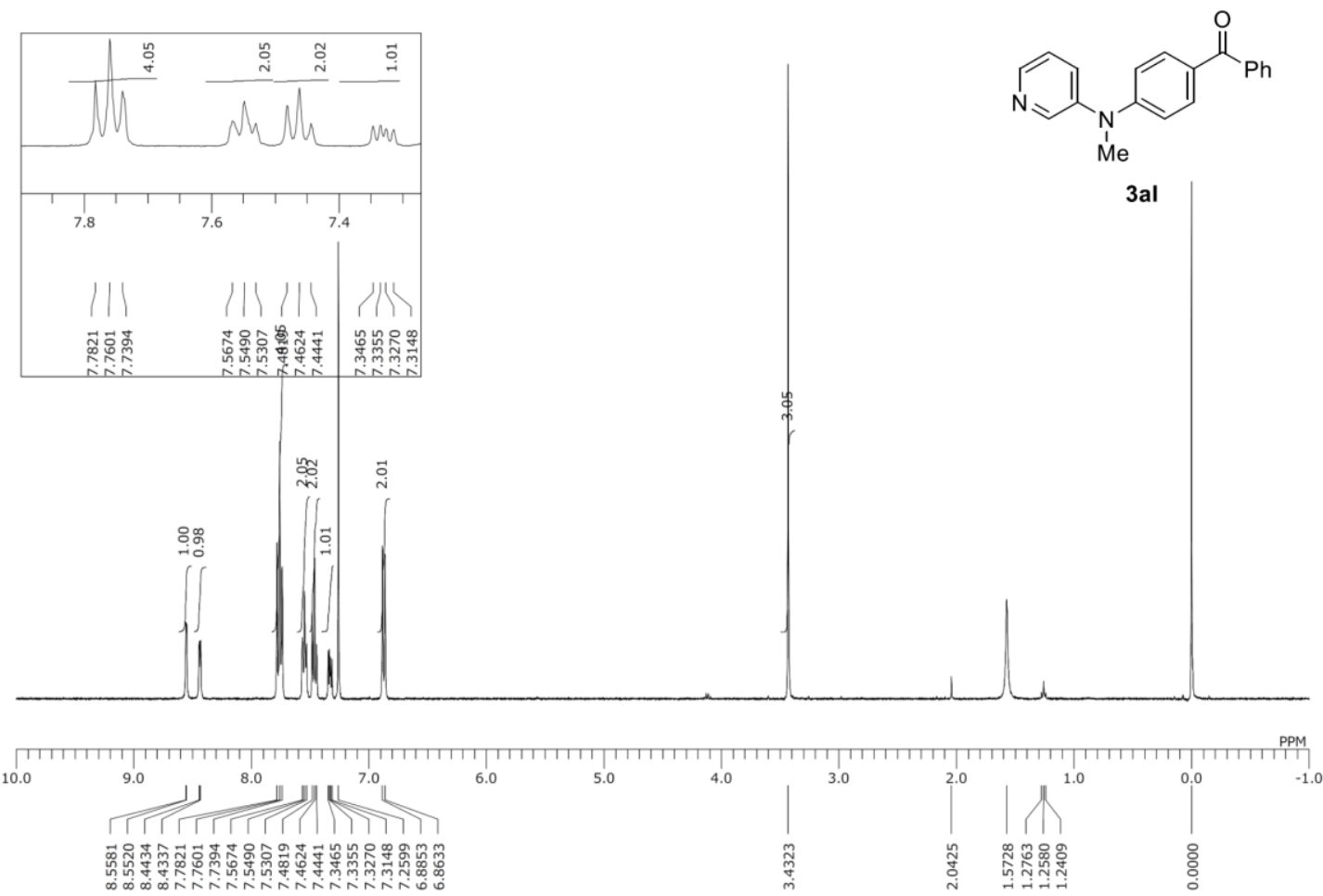

${ }^{13} \mathrm{C}$ NMR spectra of $\mathbf{3 a l}$

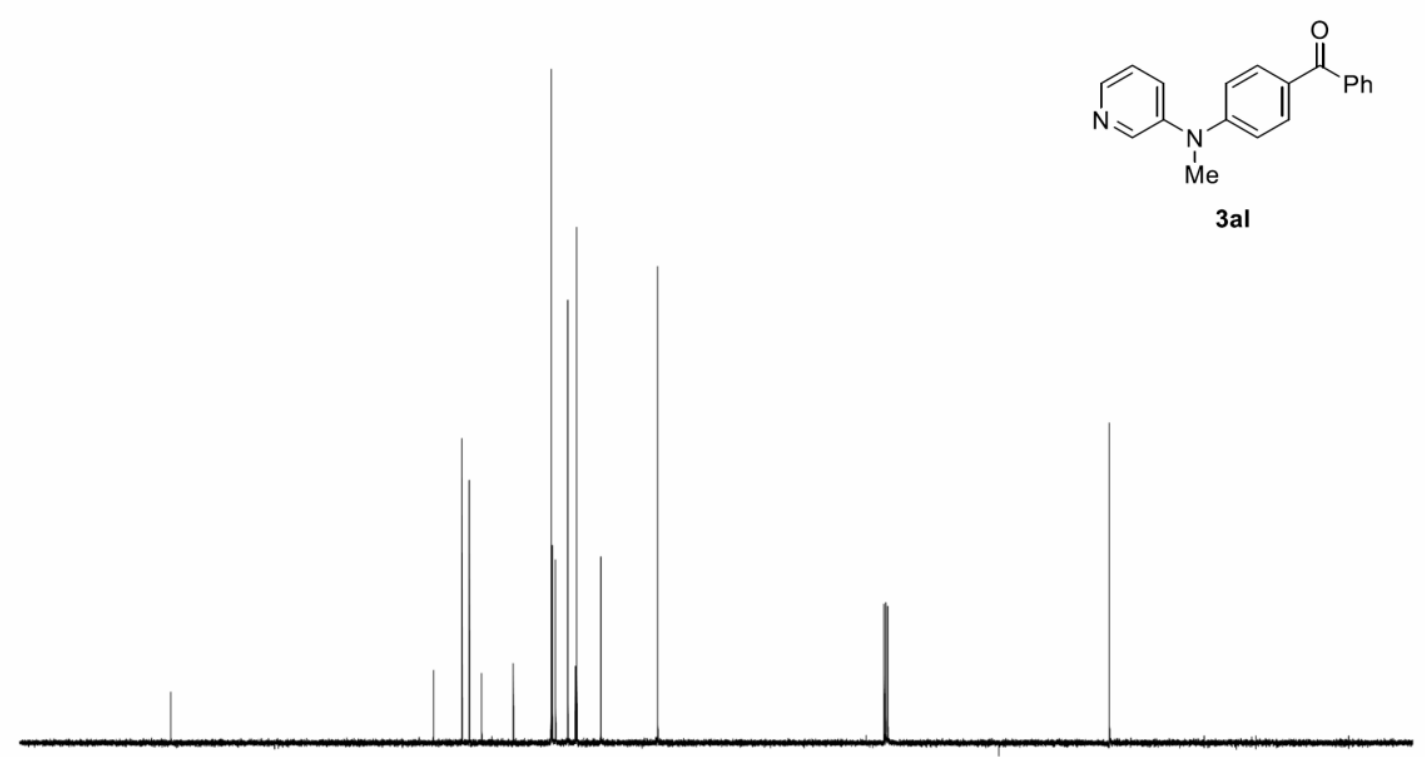

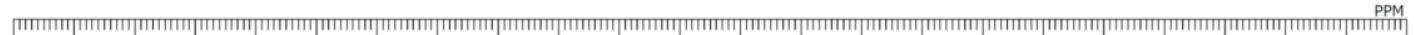

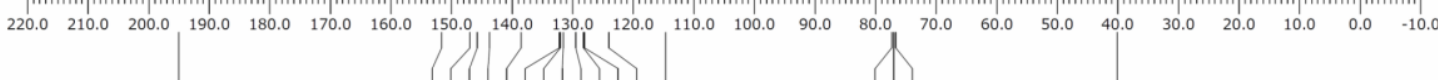

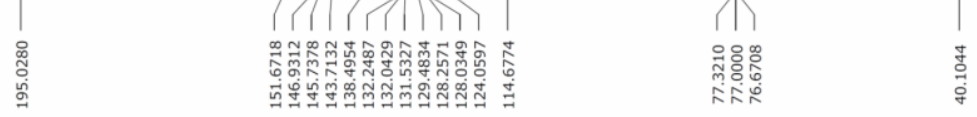


${ }^{1} \mathrm{H}$ NMR spectra of $\mathbf{3 a m}$

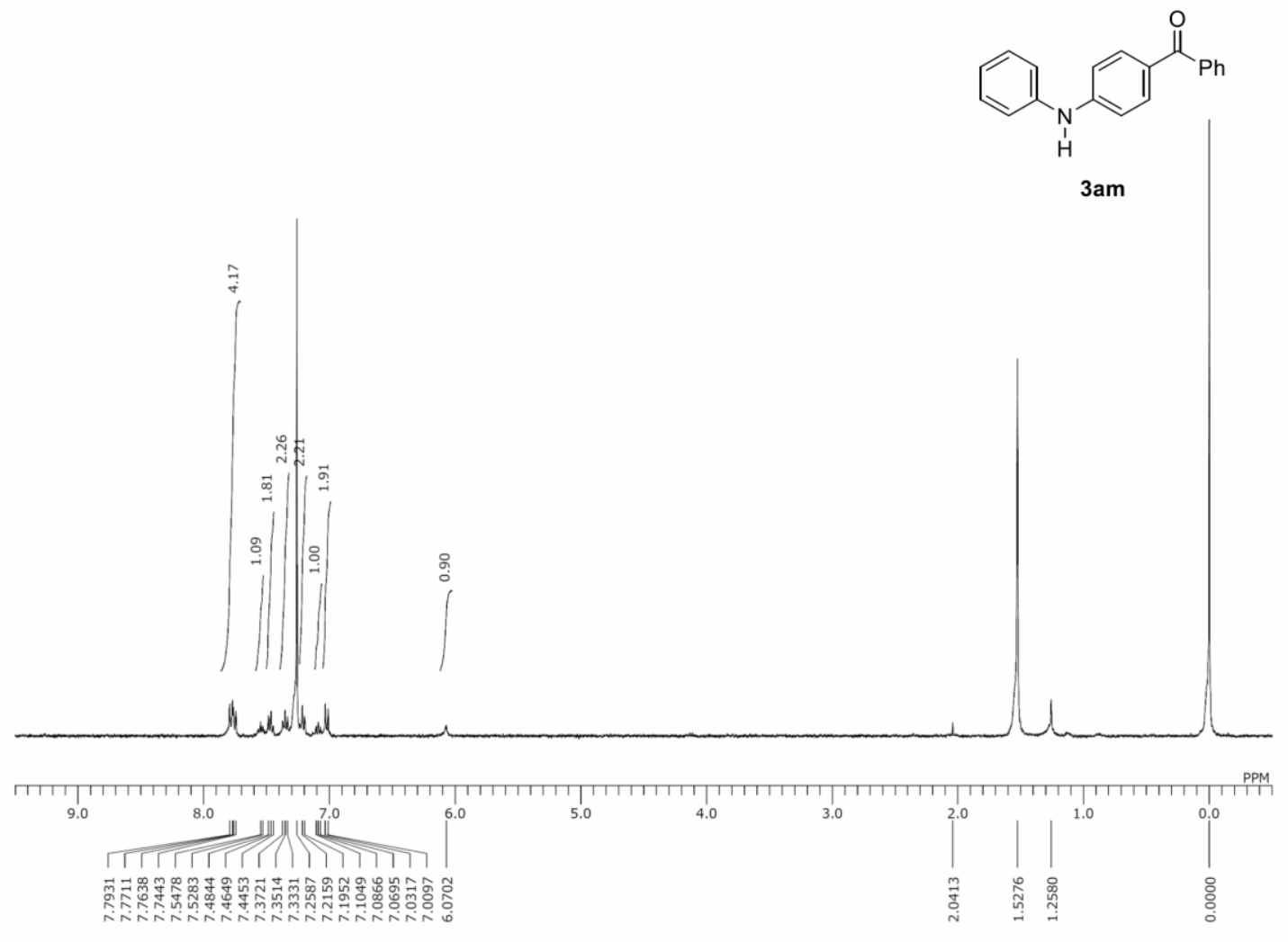

${ }^{13} \mathrm{C}$ NMR spectra of $\mathbf{3 a m}$

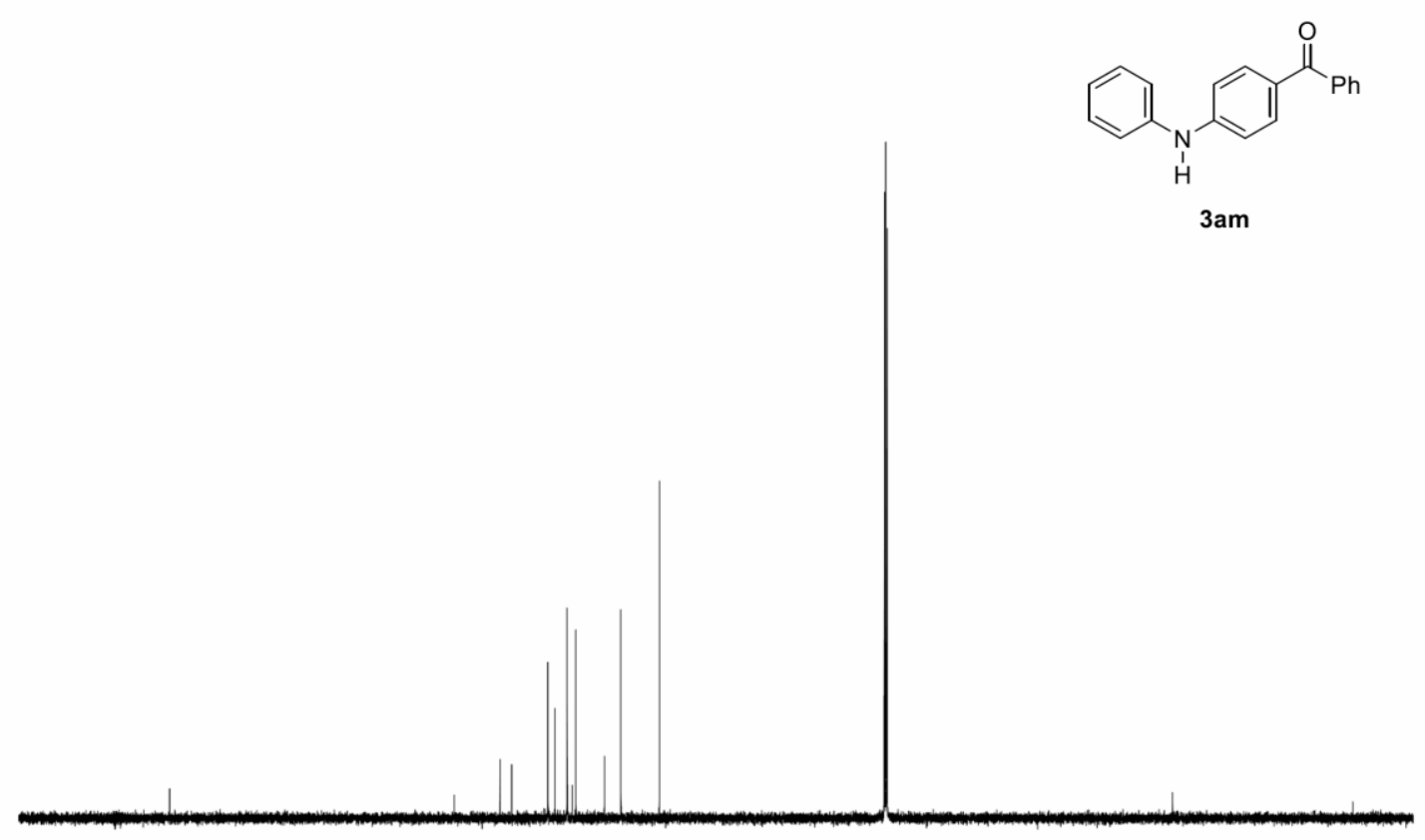

[

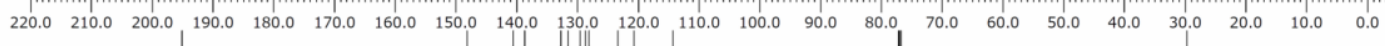

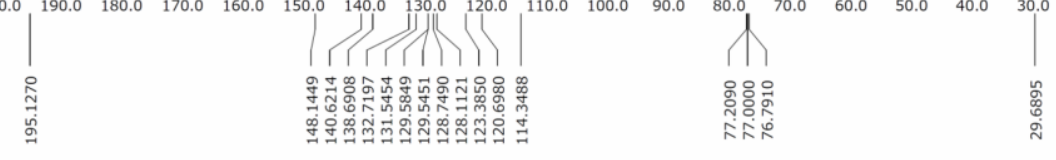


${ }^{1} \mathrm{H}$ NMR spectra of $\mathbf{3 a n}$

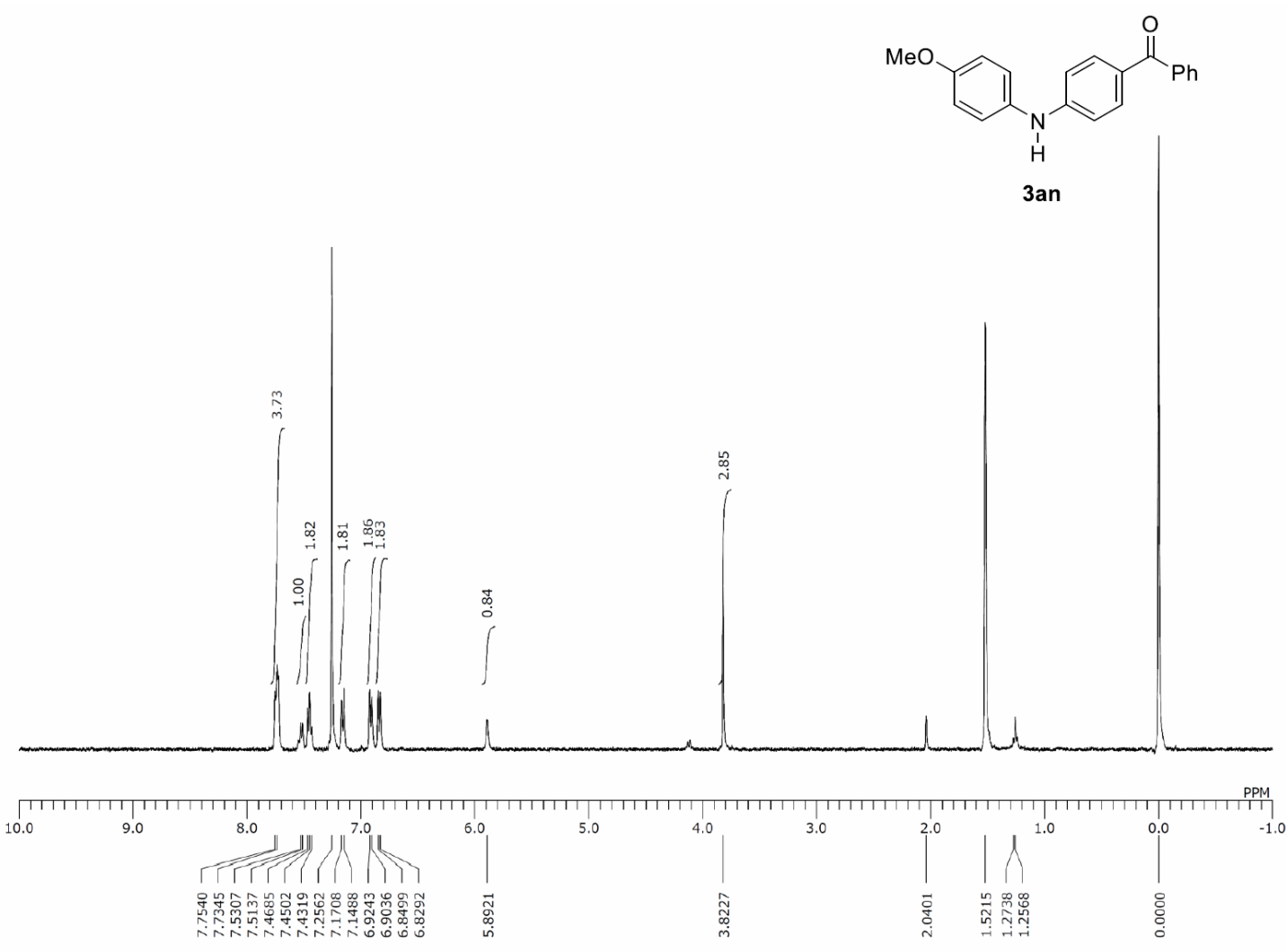

${ }^{13} \mathrm{C}$ NMR spectra of 3an

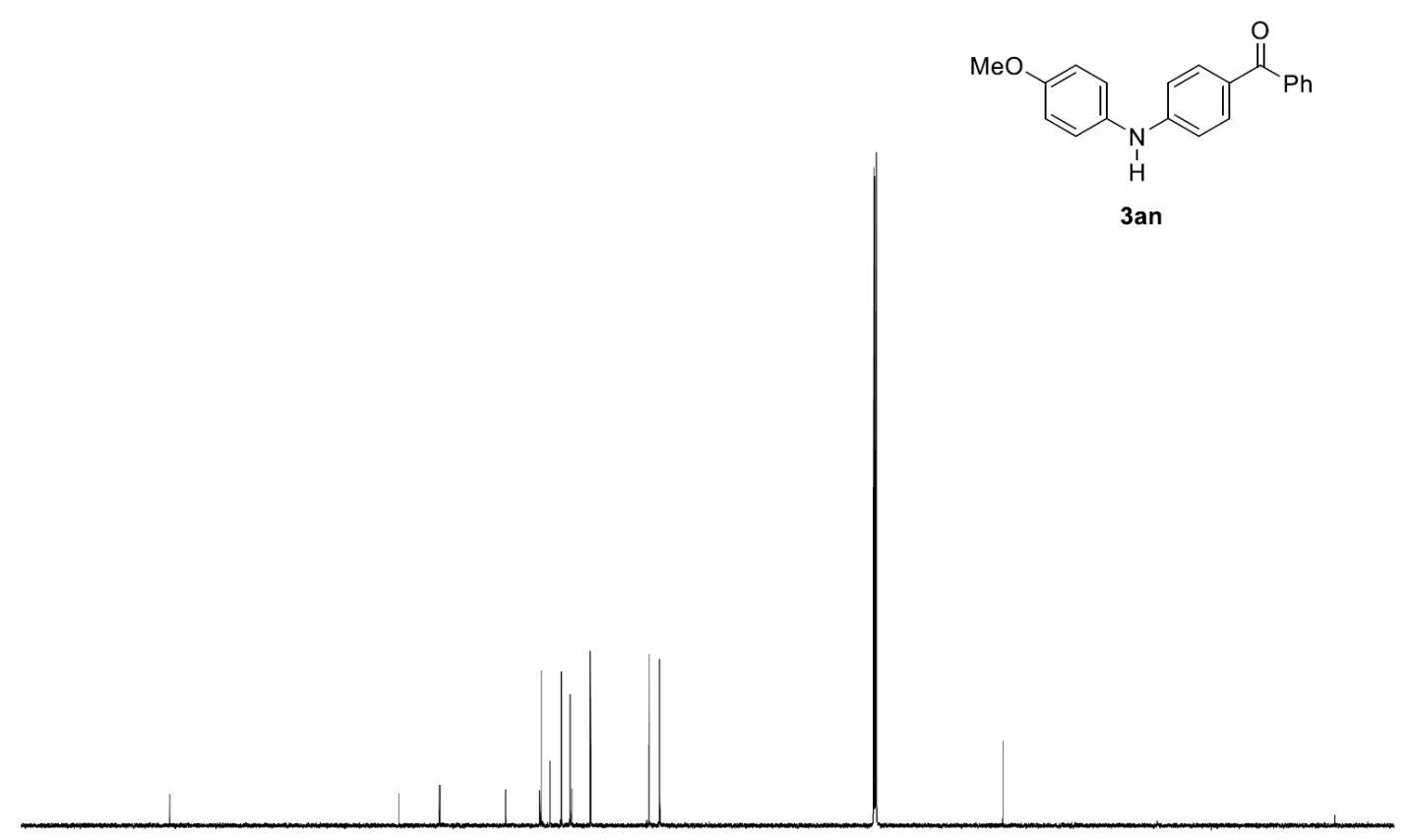

(

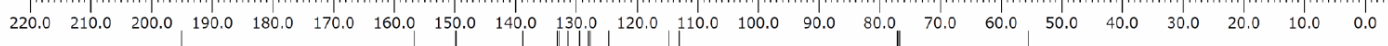

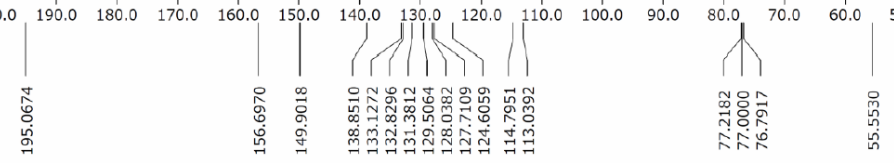


${ }^{1} \mathrm{H}$ NMR spectra of $\mathbf{3} \mathbf{b a}$

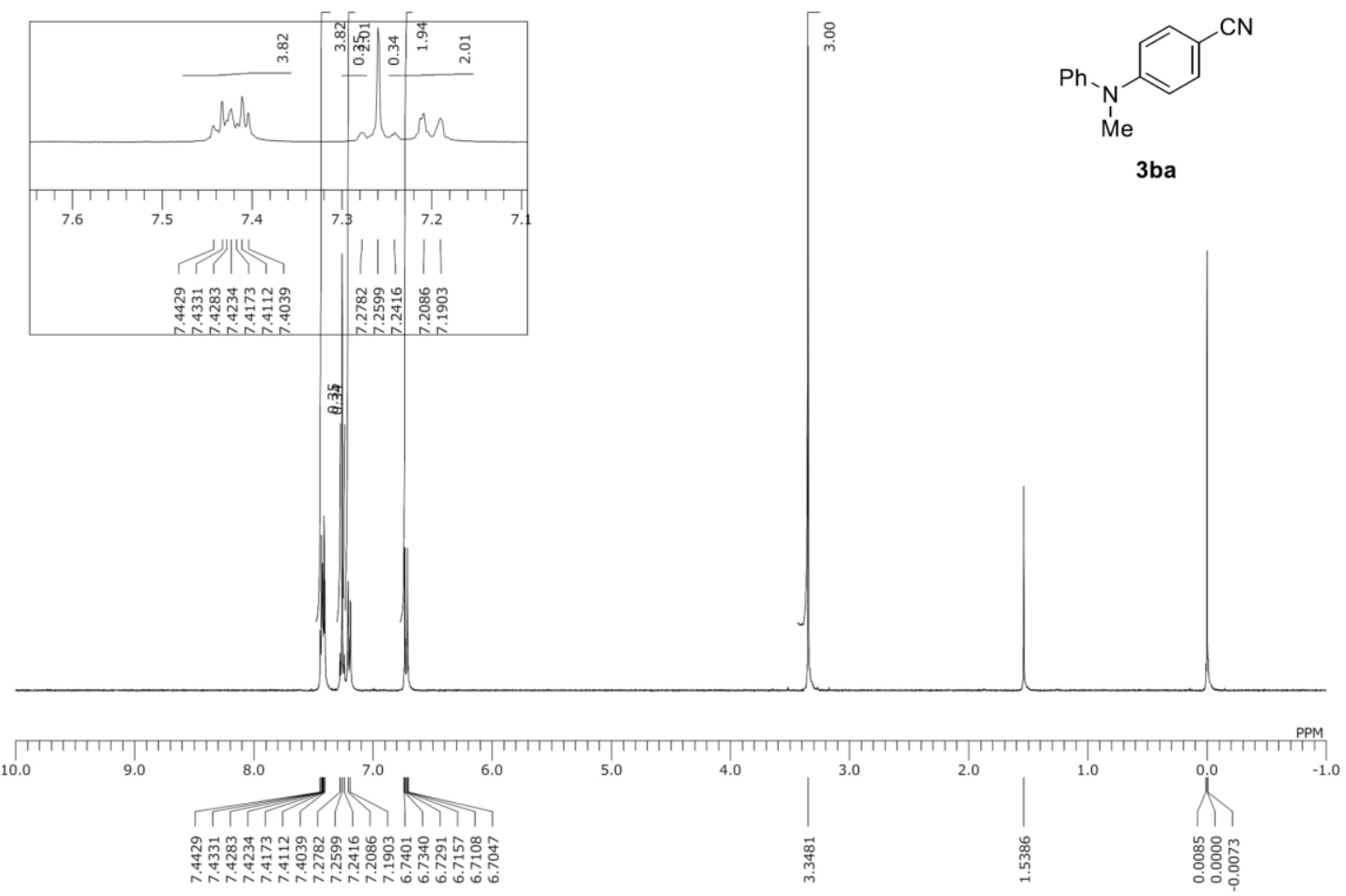

${ }^{13} \mathrm{C}$ NMR spectra of $\mathbf{3 b a}$

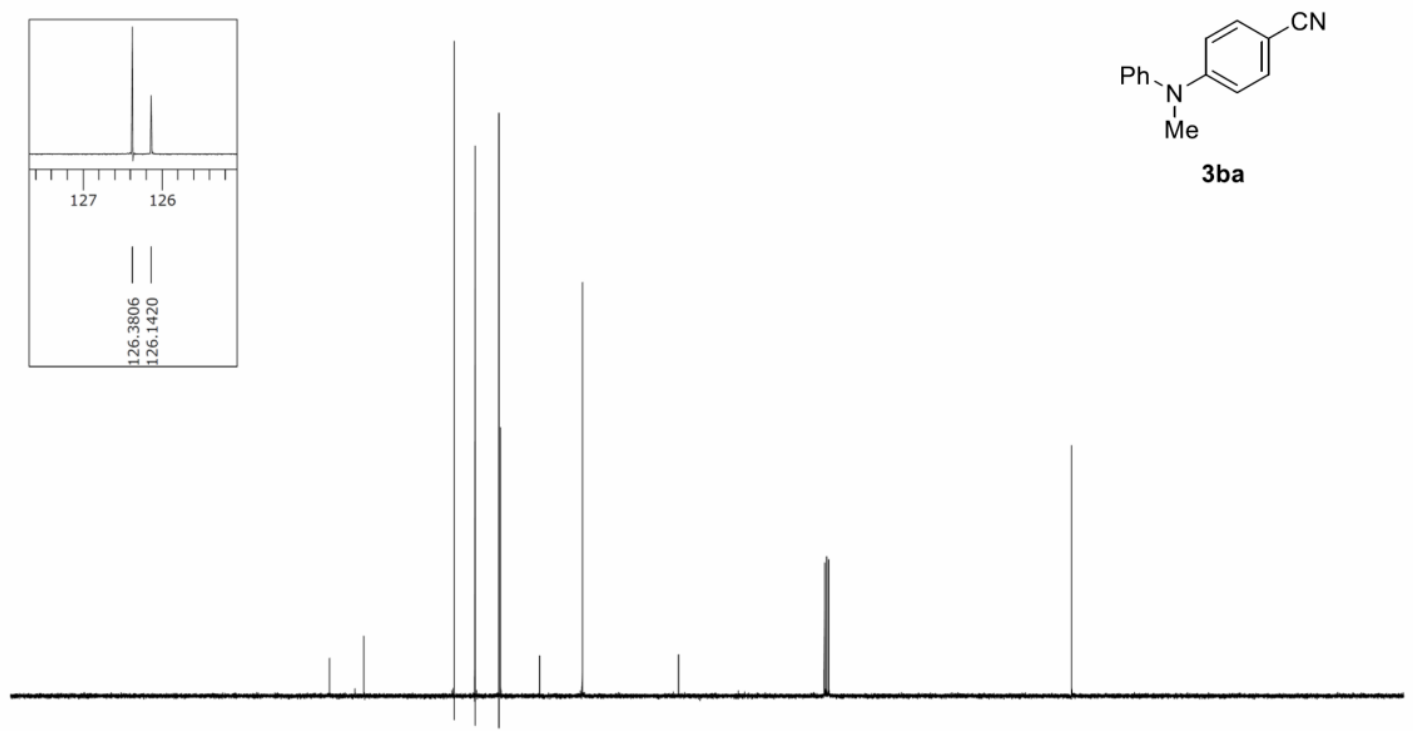

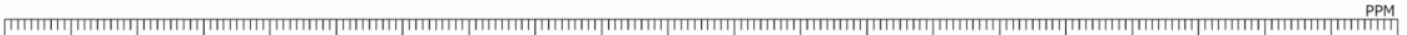

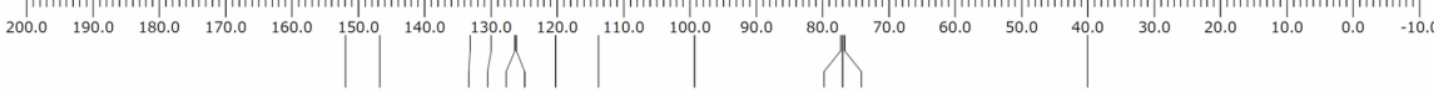

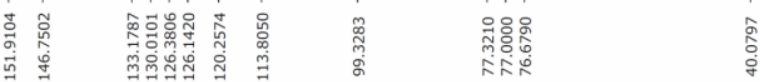



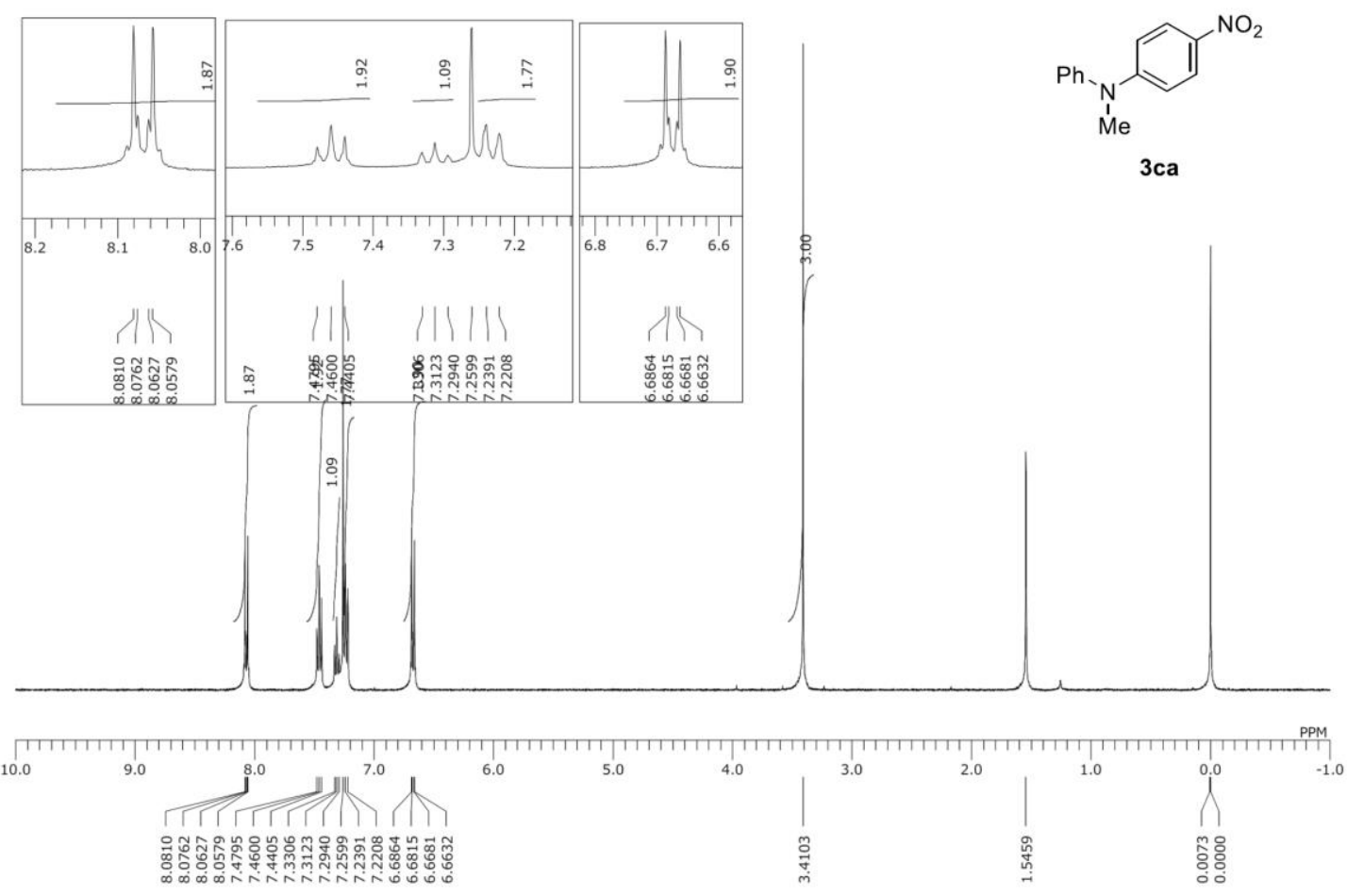

${ }^{13} \mathrm{C}$ NMR spectra of $\mathbf{3 c a}$
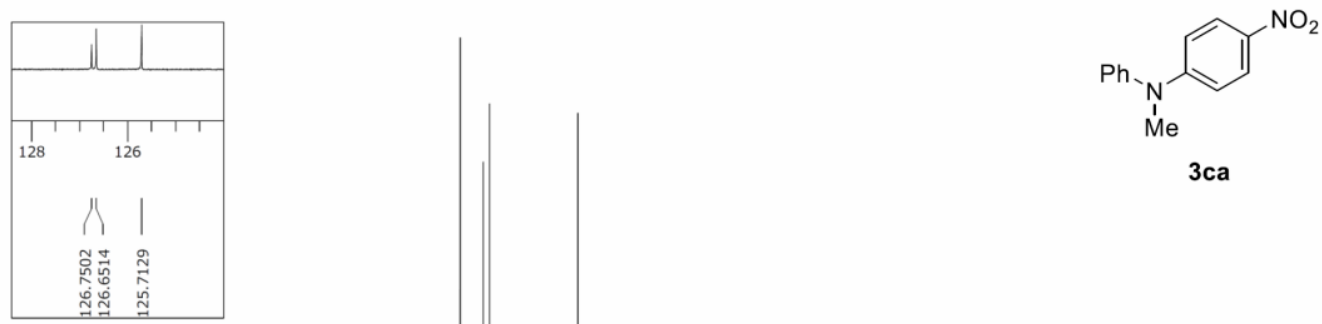

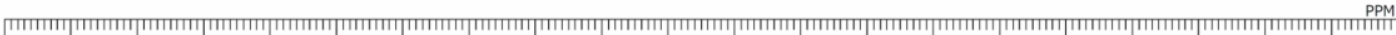

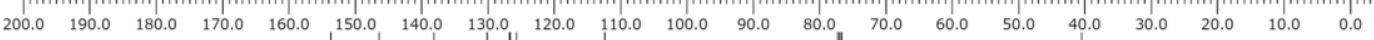

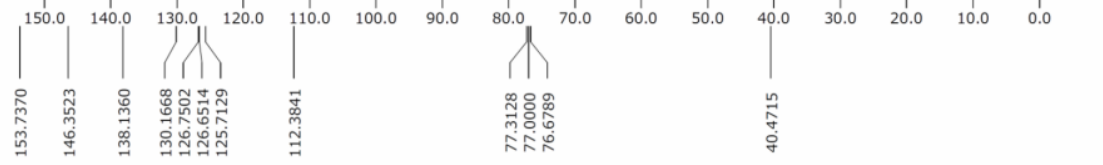



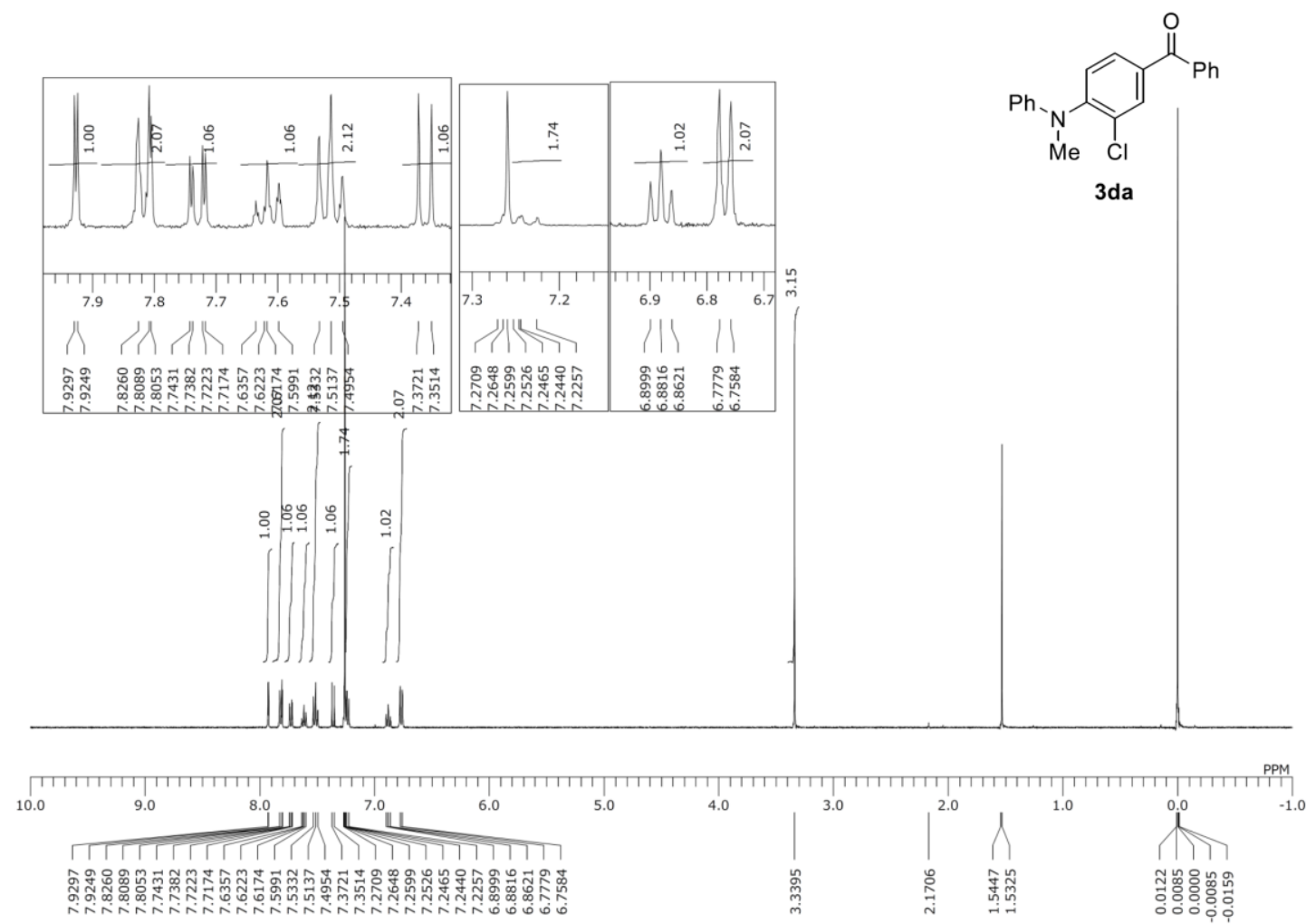

${ }^{13} \mathrm{C}$ NMR spectra of $\mathbf{3 d a}$

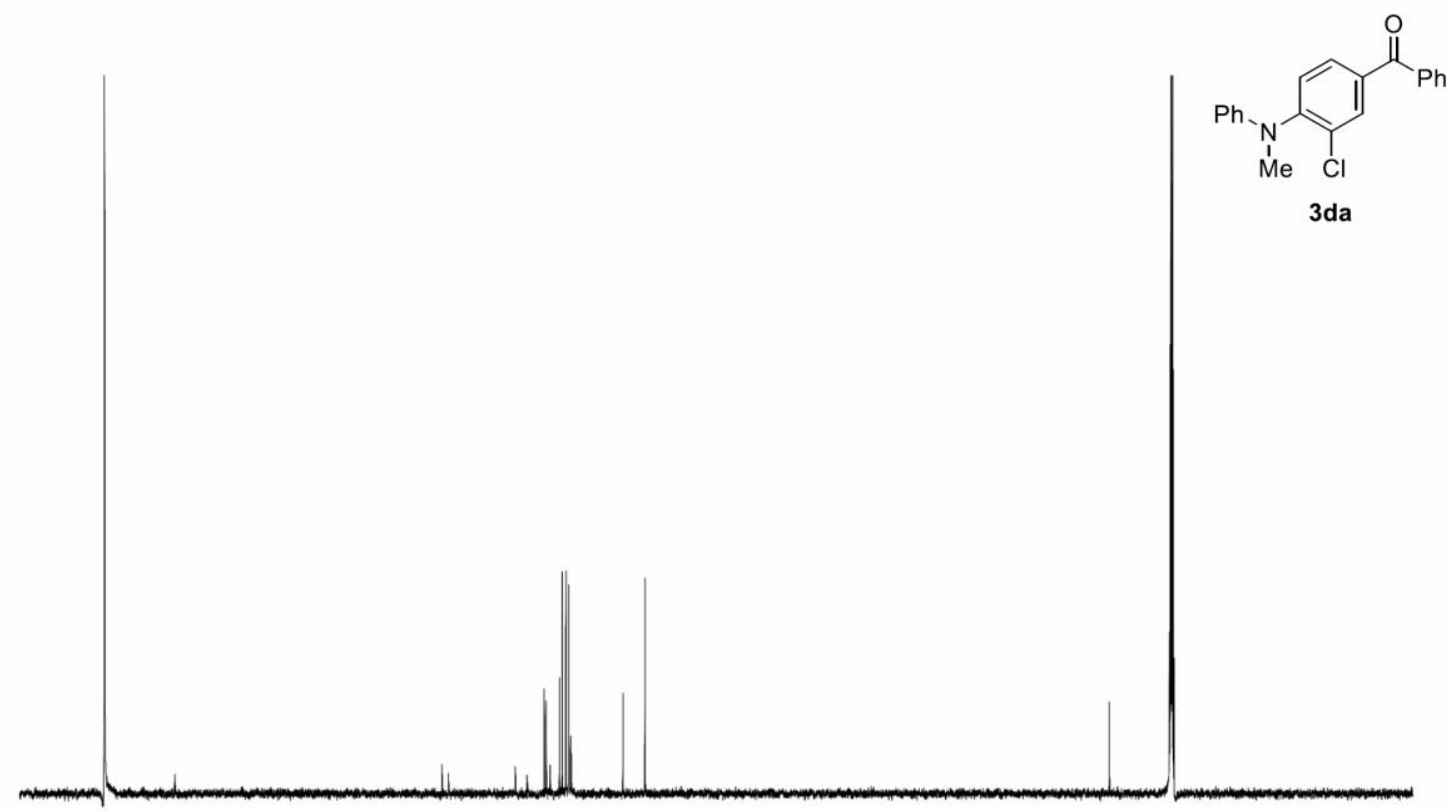

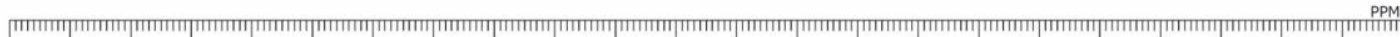
\begin{tabular}{l|llllllllllllllllllllll}
220.0 & 210.0 & 200.0 & 190.0 & 180.0 & 170.0 & 160.0 & 150.0 & 140.0 & 130.0 & 120.0 & 110.0 & 100.0 & 90.0 & 80.0 & 70.0 & 60.0 & 50.0 & 40.0 & 30.0 & 20.0 & 10.0 & 0.0
\end{tabular}

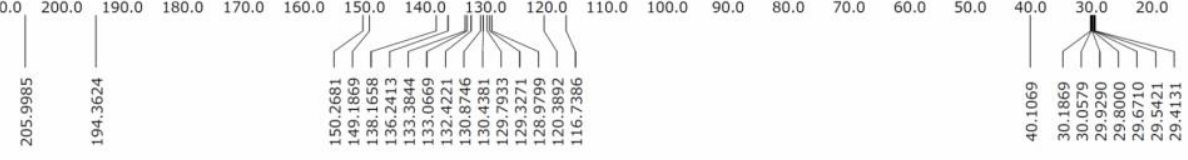


${ }^{1} \mathrm{H}$ NMR spectra of $\mathbf{3 e g}$
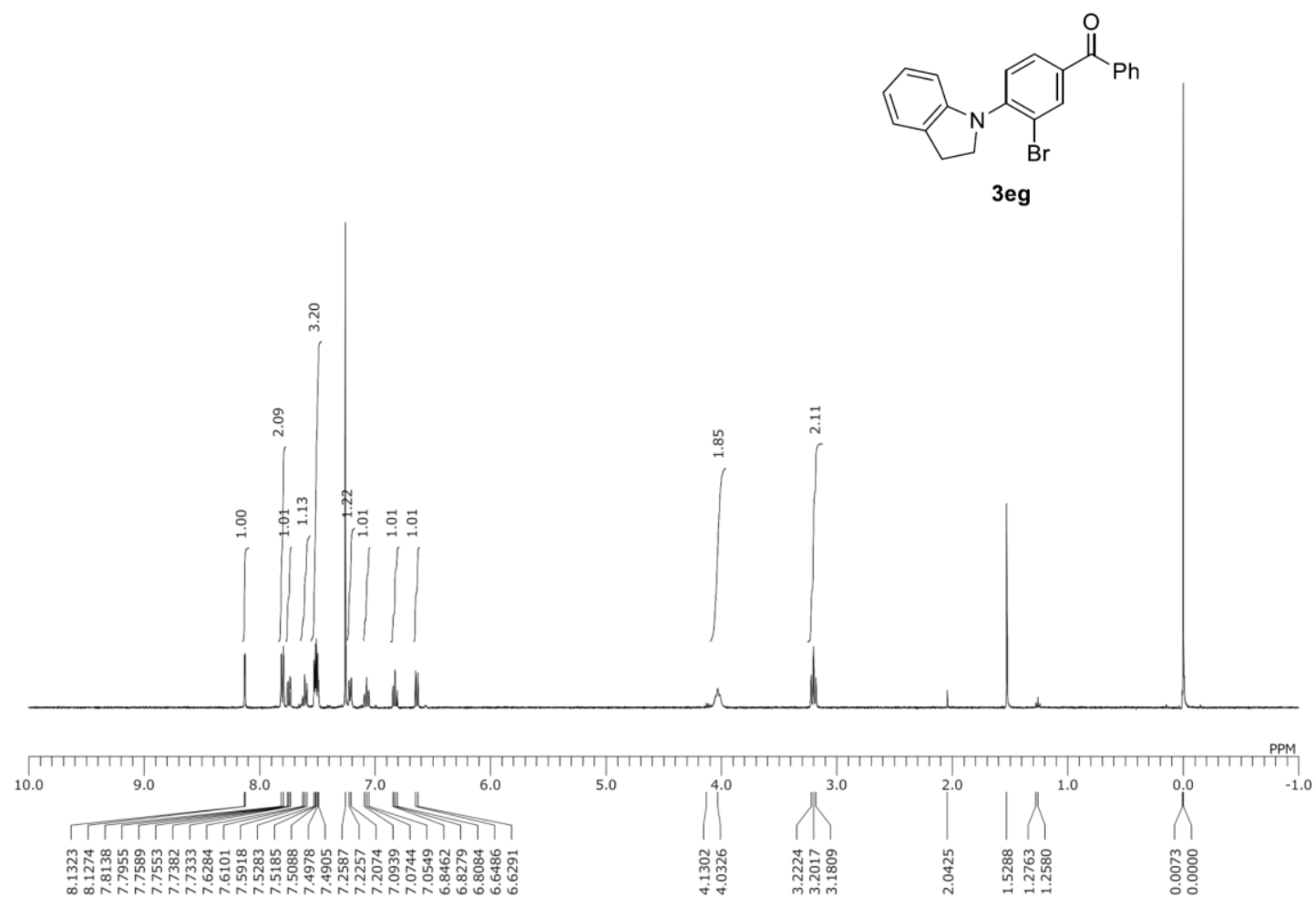

${ }^{13} \mathrm{C}$ NMR spectra of $\mathbf{3 e g}$
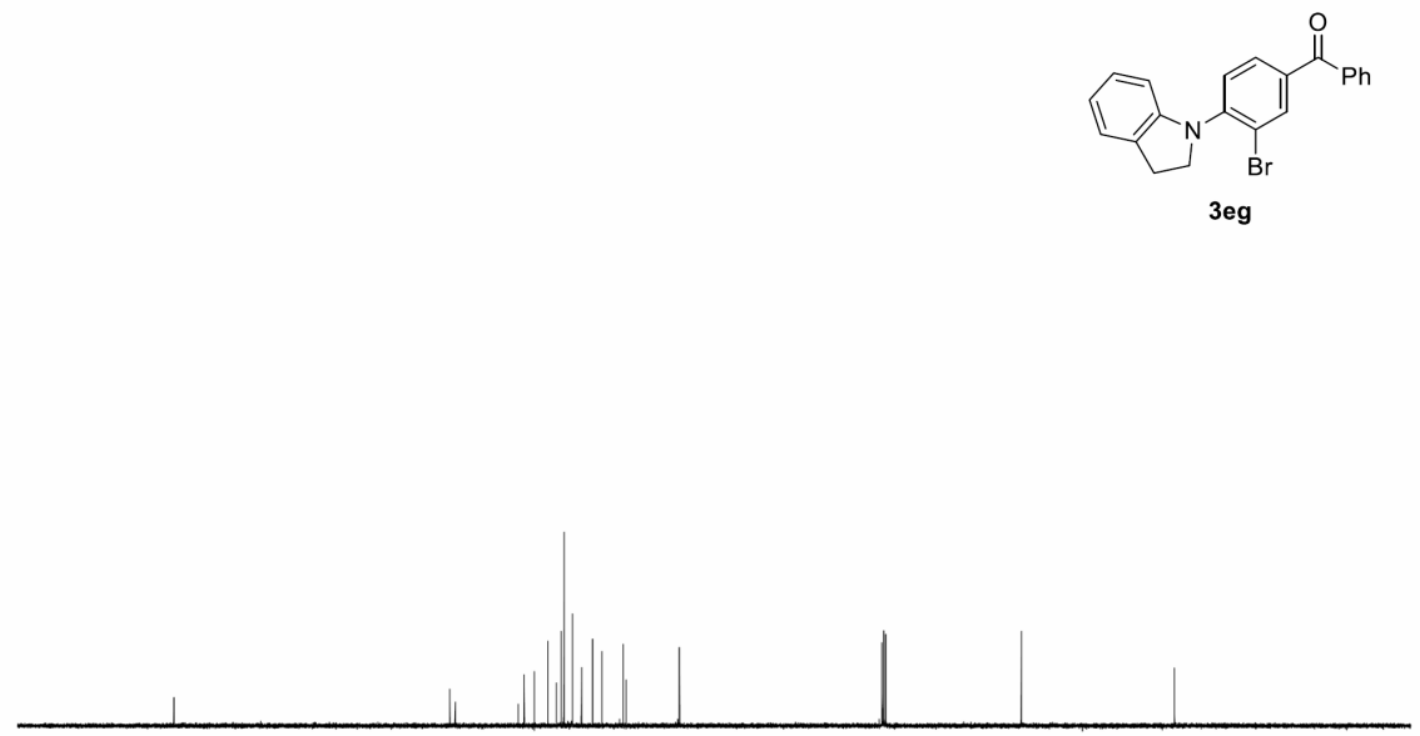

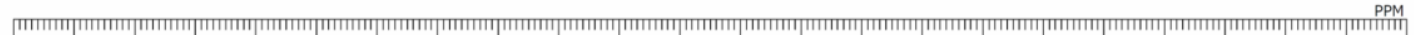

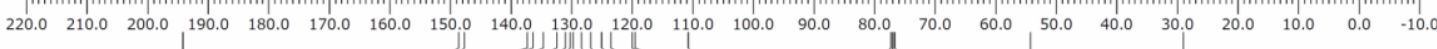

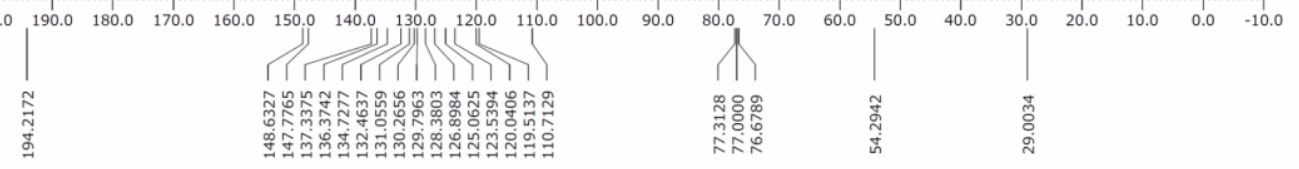


${ }^{1} \mathrm{H}$ NMR spectra of $\mathbf{3} \mathbf{g}$

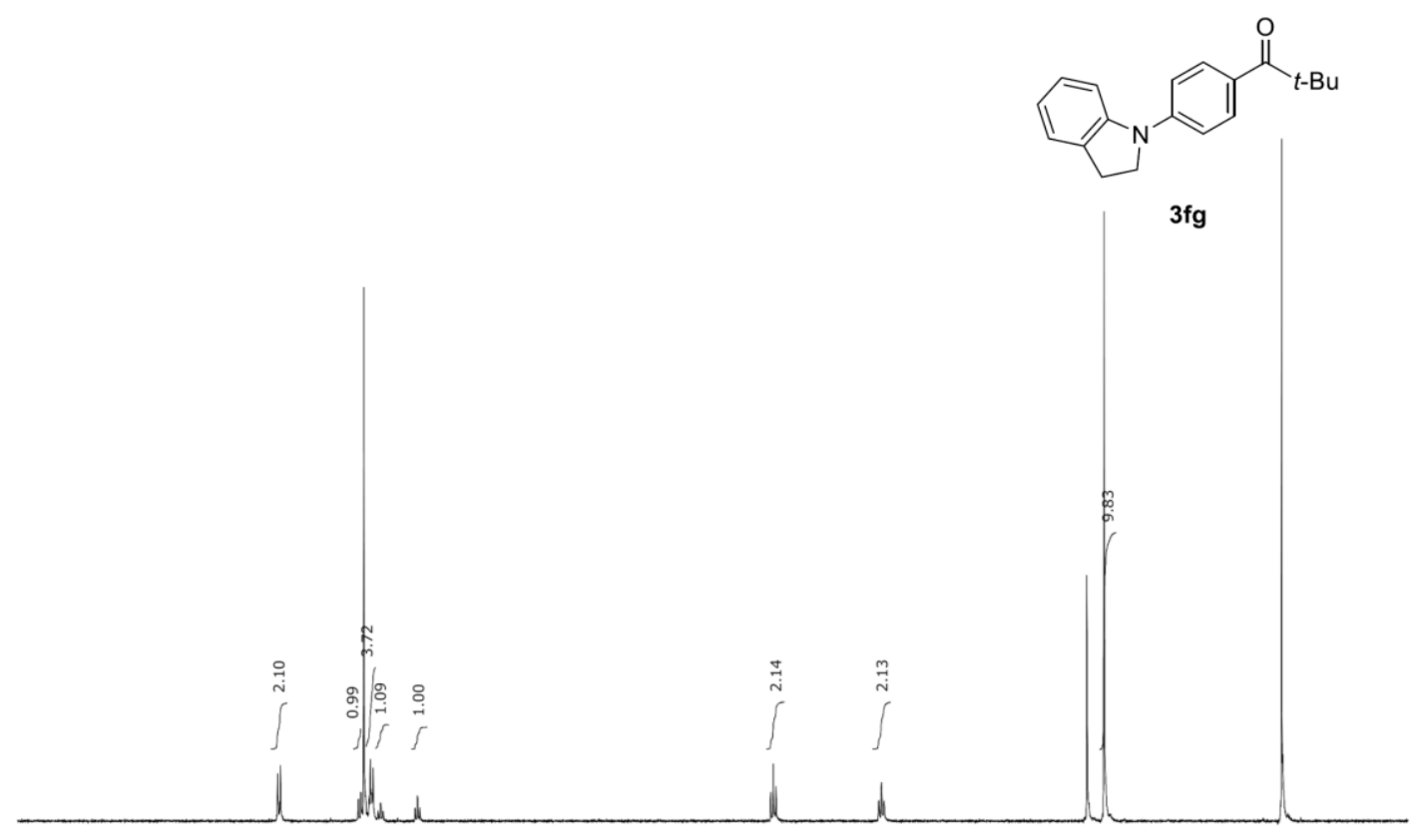

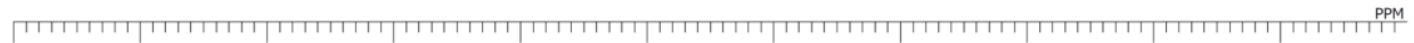

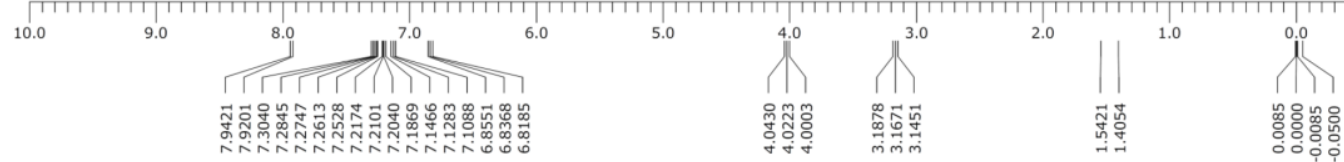

${ }^{13} \mathrm{C}$ NMR spectra of $\mathbf{3 f g}$

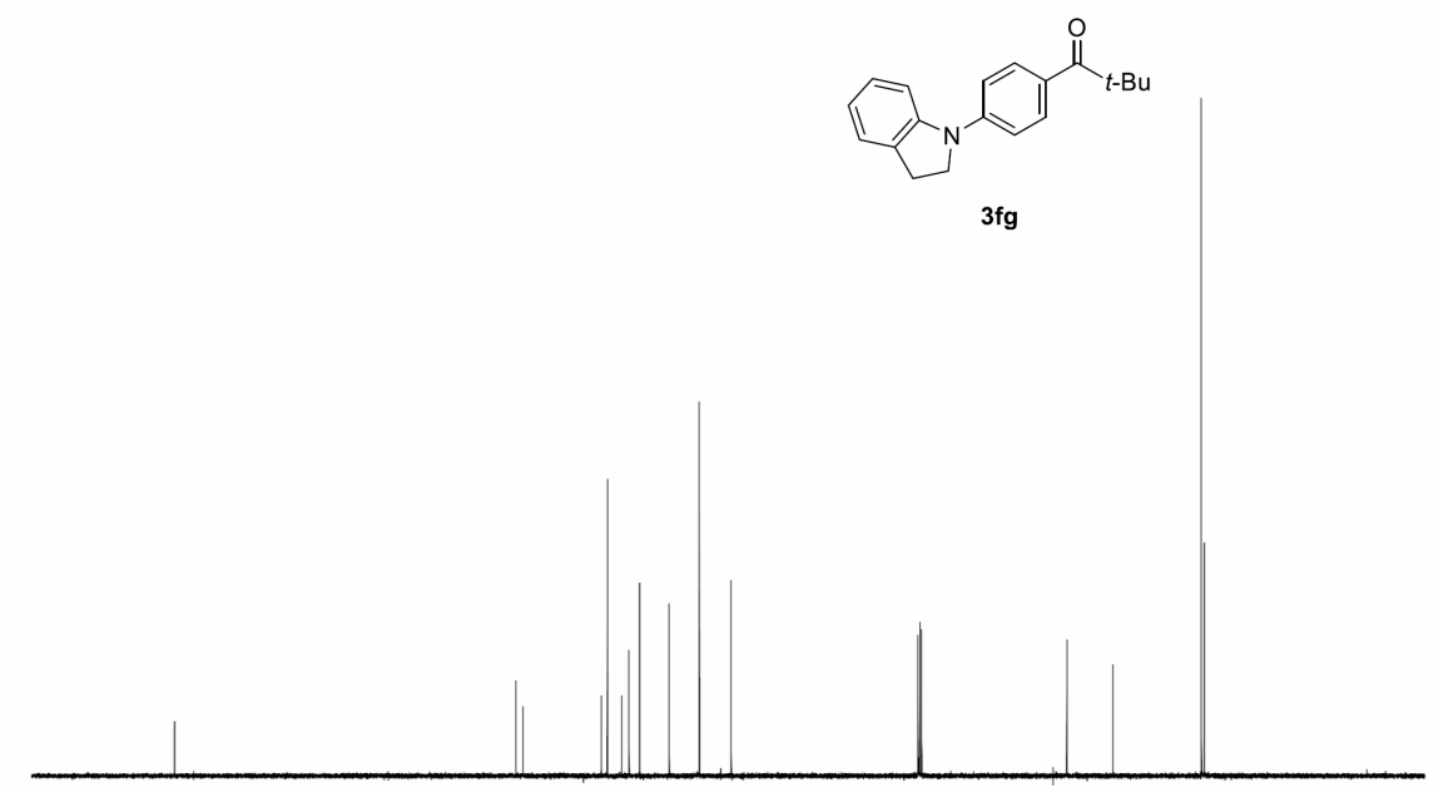

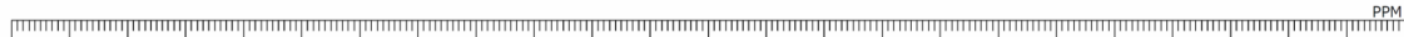

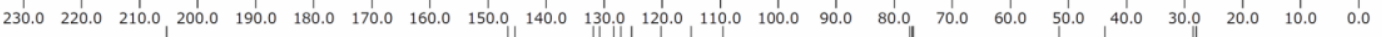

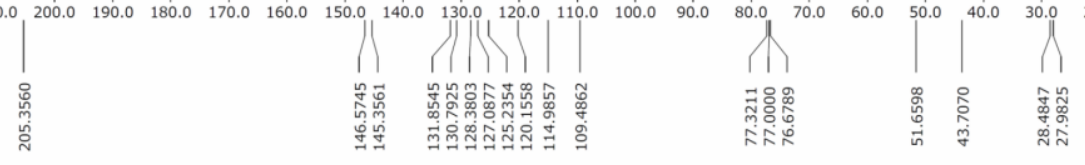


${ }^{1} \mathrm{H}$ NMR spectra of $\mathbf{3 g g}$
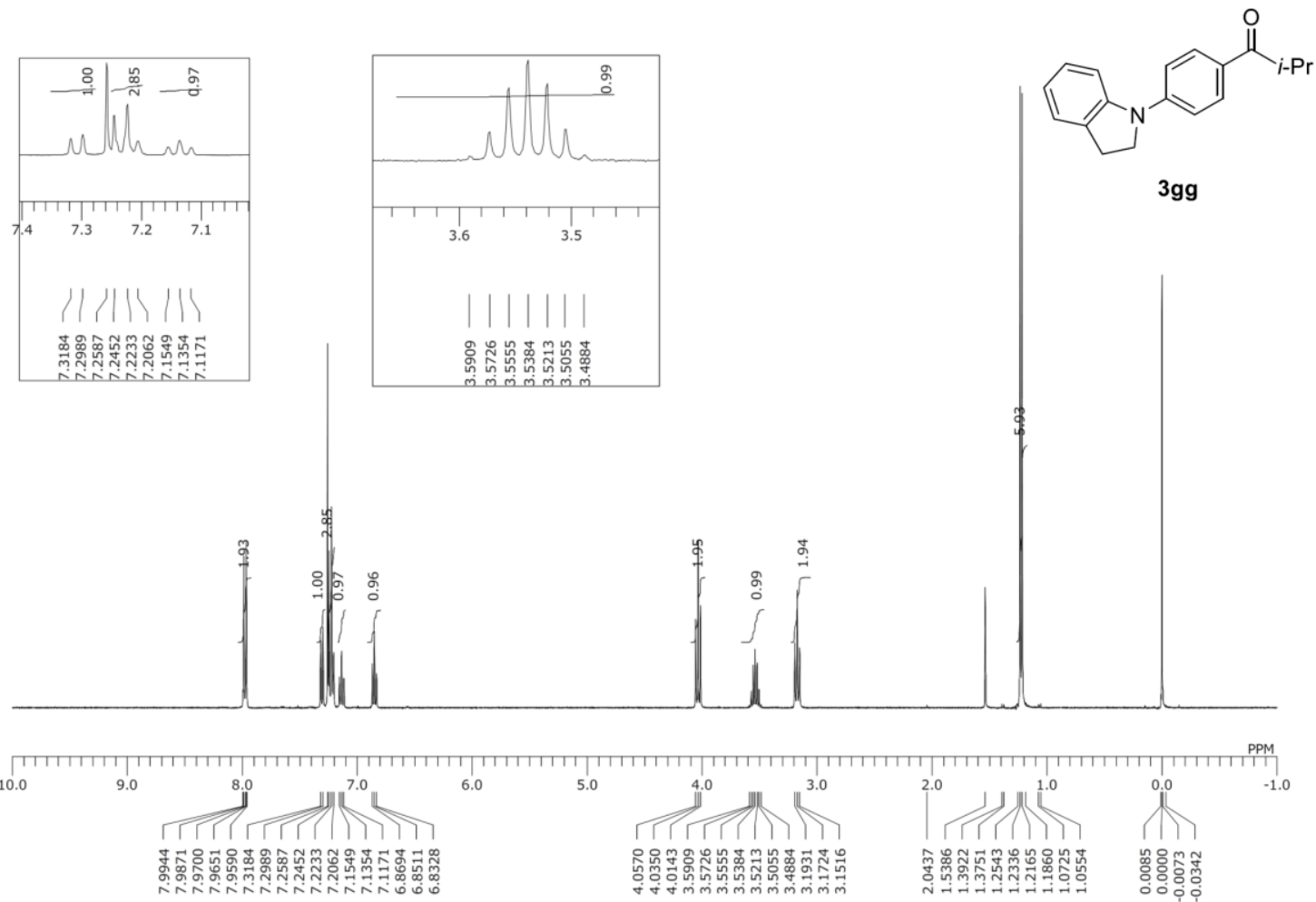

${ }^{13} \mathrm{C}$ NMR spectra of $\mathbf{3 g g}$

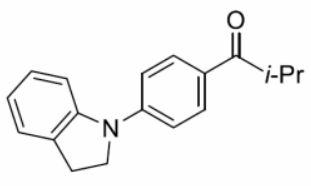

3gg

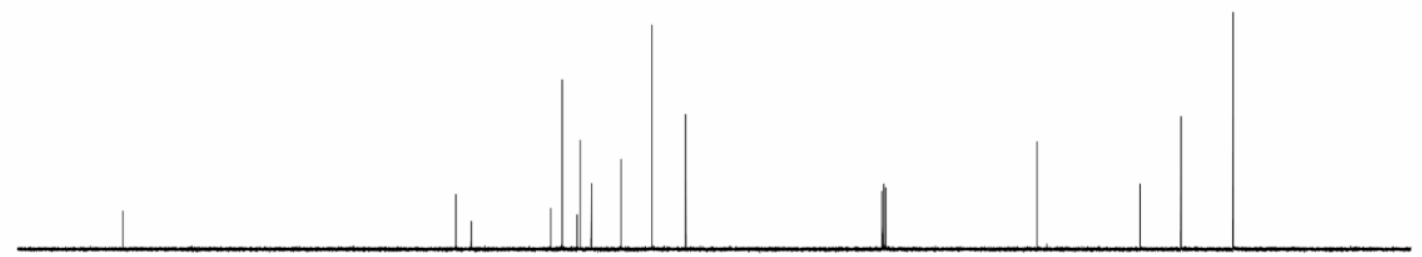

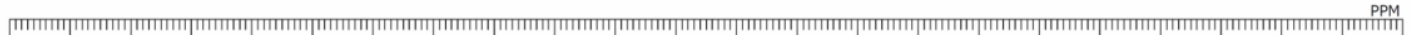
$\begin{array}{llllllllllllllllllllllll}220.0 & 210.0 & 200.0 & 190.0 & 180.0 & 170.0 & 160.0 & 150.0 & 140.0 & 130.0 & 120.0 & 110.0 & 100.0 & 90.0 & 80.0 & 70.0 & 60.0 & 50.0 & 40.0 & 30.0 & 20.0 & 10.0 & 0.0 & -10.0\end{array}$

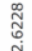

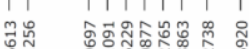

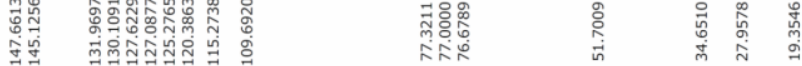


${ }^{1} \mathrm{H}$ NMR spectra of $\mathbf{3 h a}$

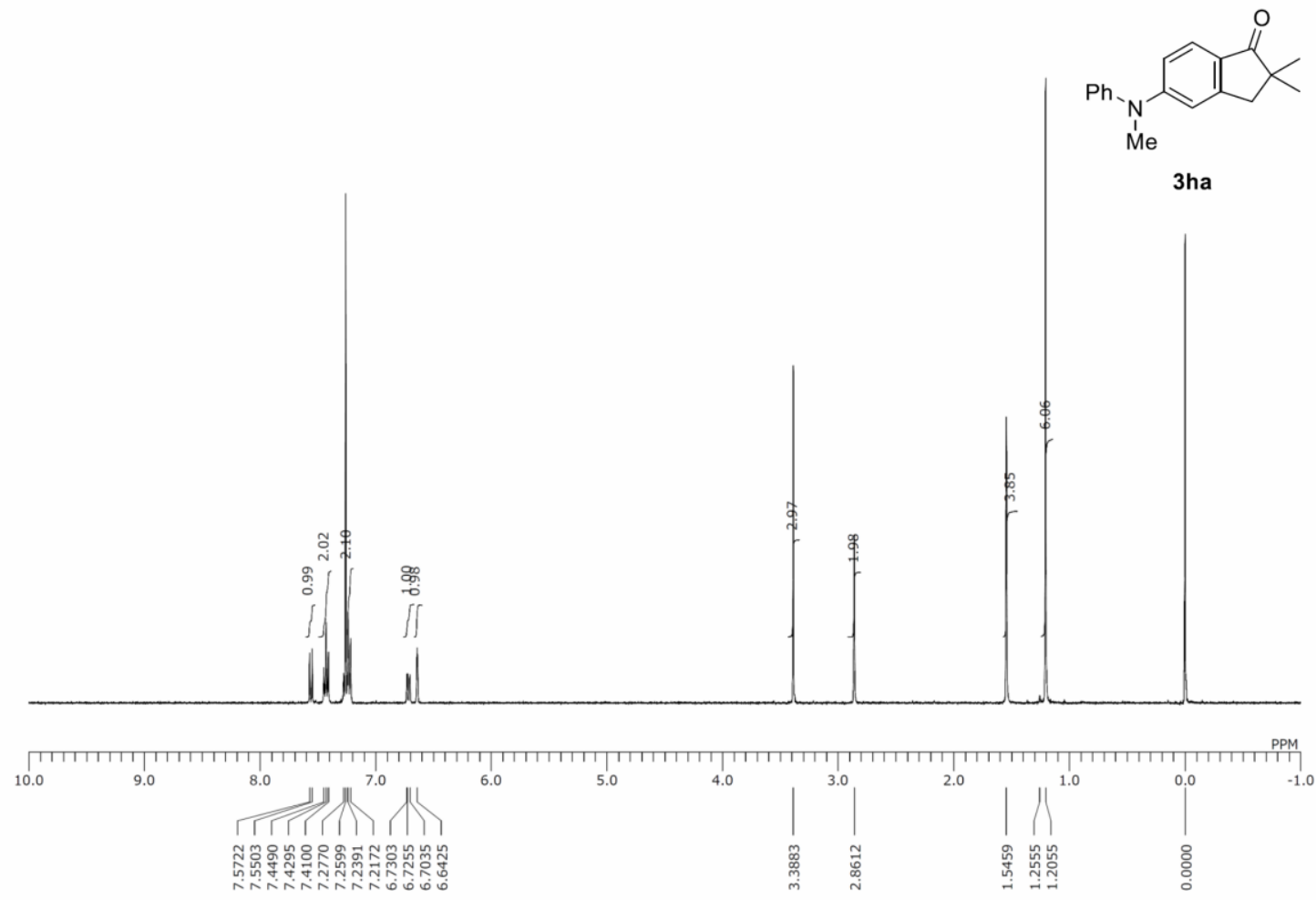

${ }^{13} \mathrm{C}$ NMR spectra of $\mathbf{3 h a}$

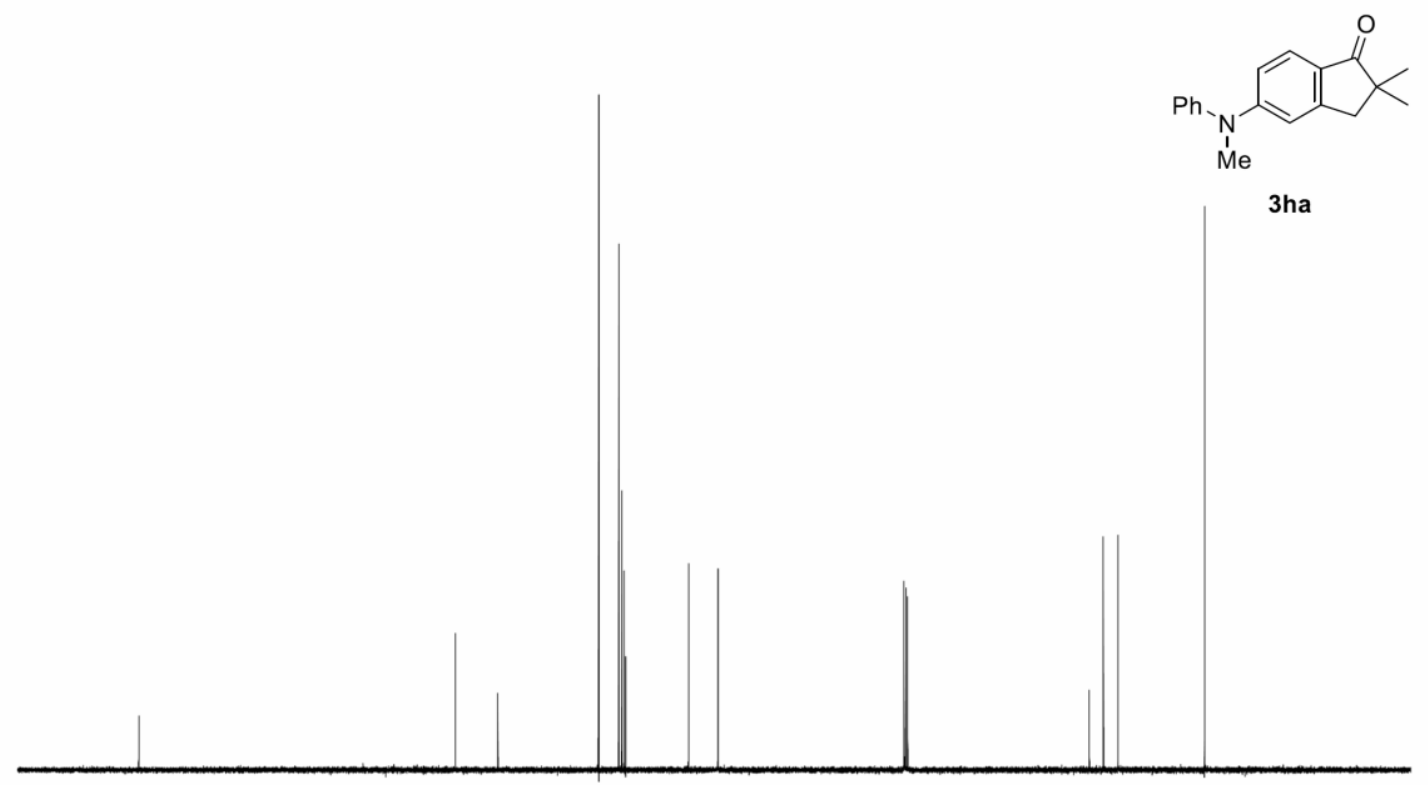

[ $\begin{array}{llllllllllllllllllllllllllll}230.0 & 220.0 & 210.0 & 200.0 & 190.0 & 180.0 & 170.0 & 160.0 & 150.0 & 140.0 & 130.0 & 120.0 & 110.0 & 100.0 & 90.0 & 80.0 & 70.0 & 60.0 & 50.0 & 40.0 & 30.0 & 20.0 & 10.0 & 0.0\end{array}$

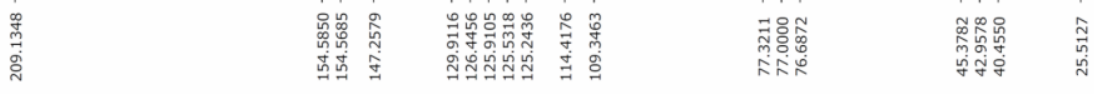


${ }^{1} \mathrm{H}$ NMR spectra of $\mathbf{3 j a}$

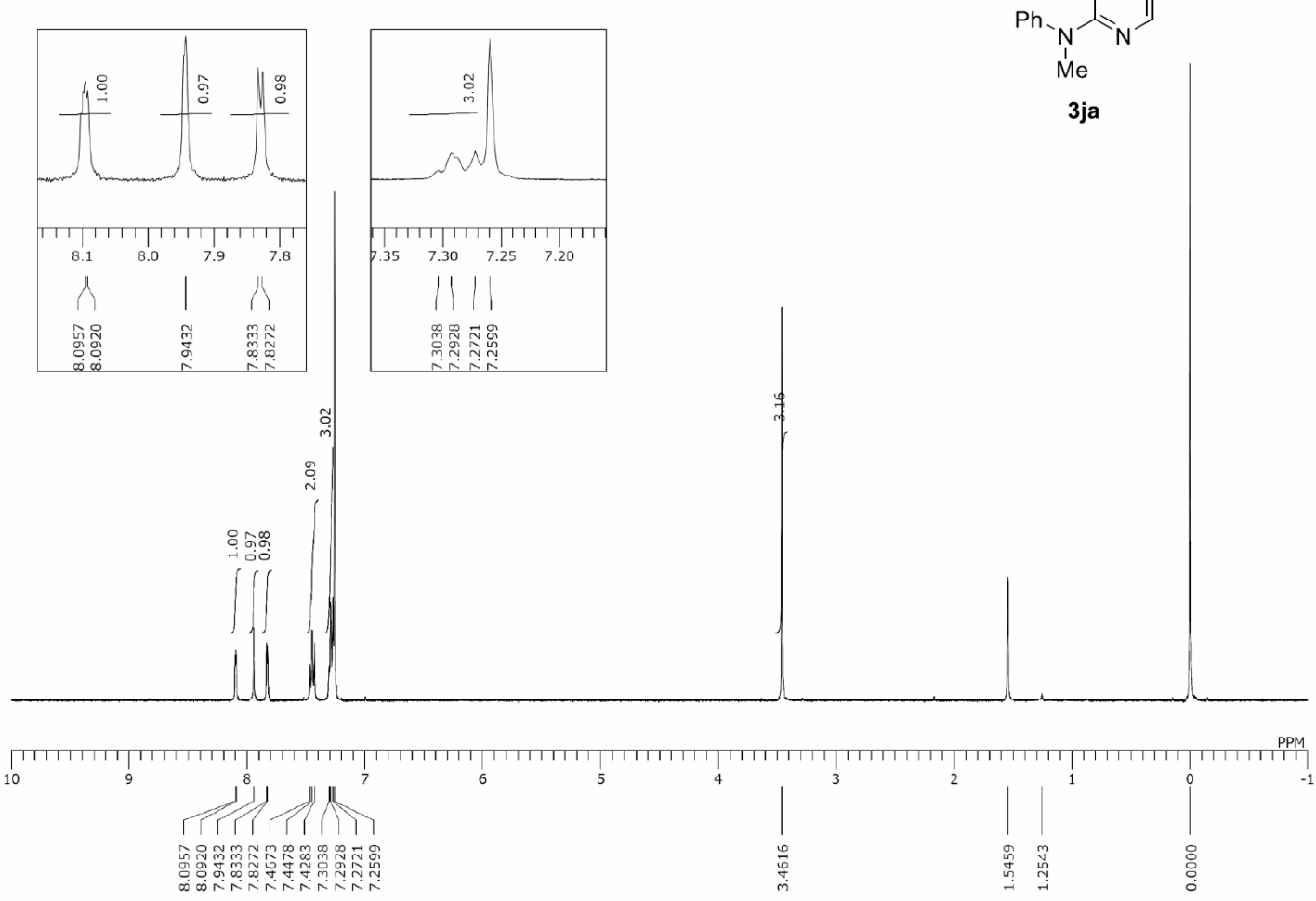

${ }^{13} \mathrm{C}$ NMR spectra of $\mathbf{3 j a}$
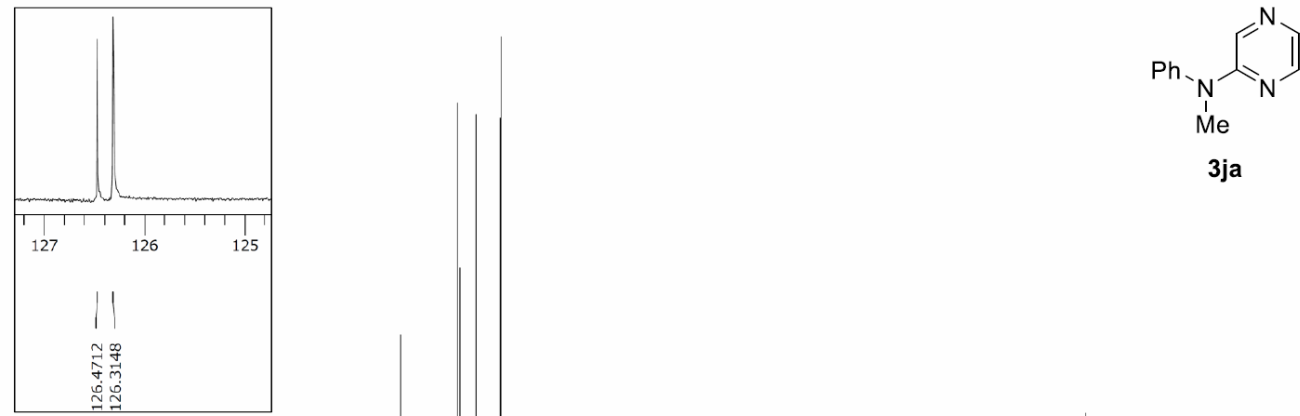

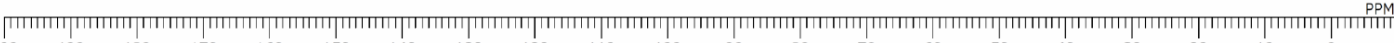
$\begin{array}{lllll}200 & 190 & 180 & 170 & 160\end{array}$

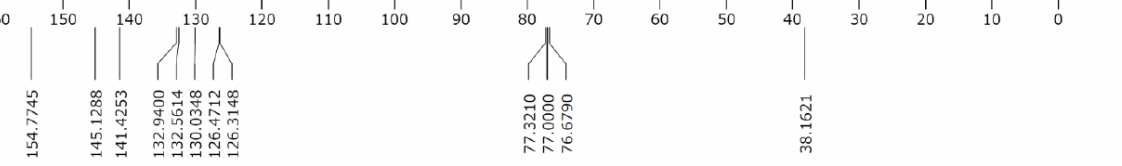



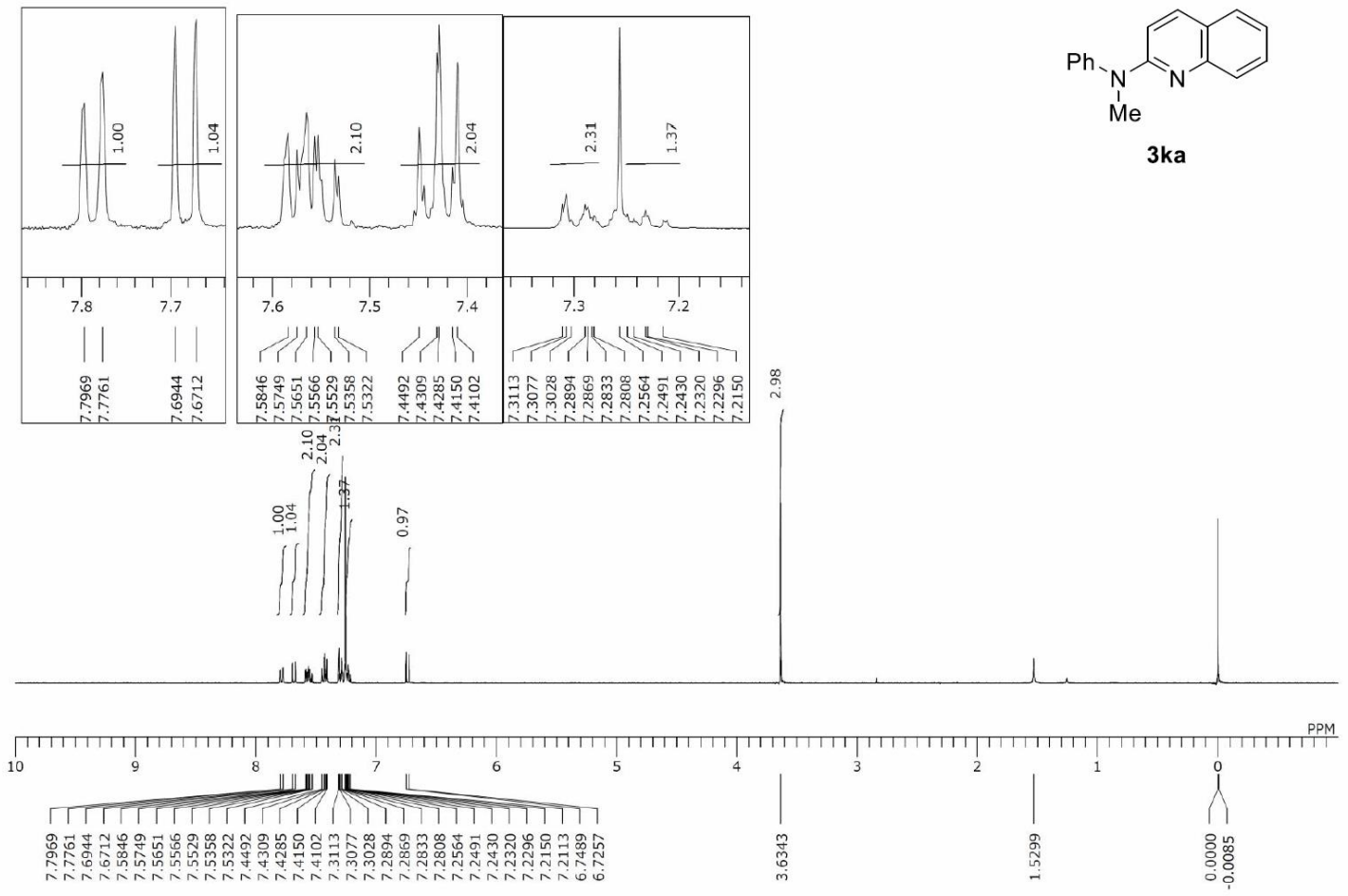

${ }^{13} \mathrm{C}$ NMR spectra of $\mathbf{3 k a}$
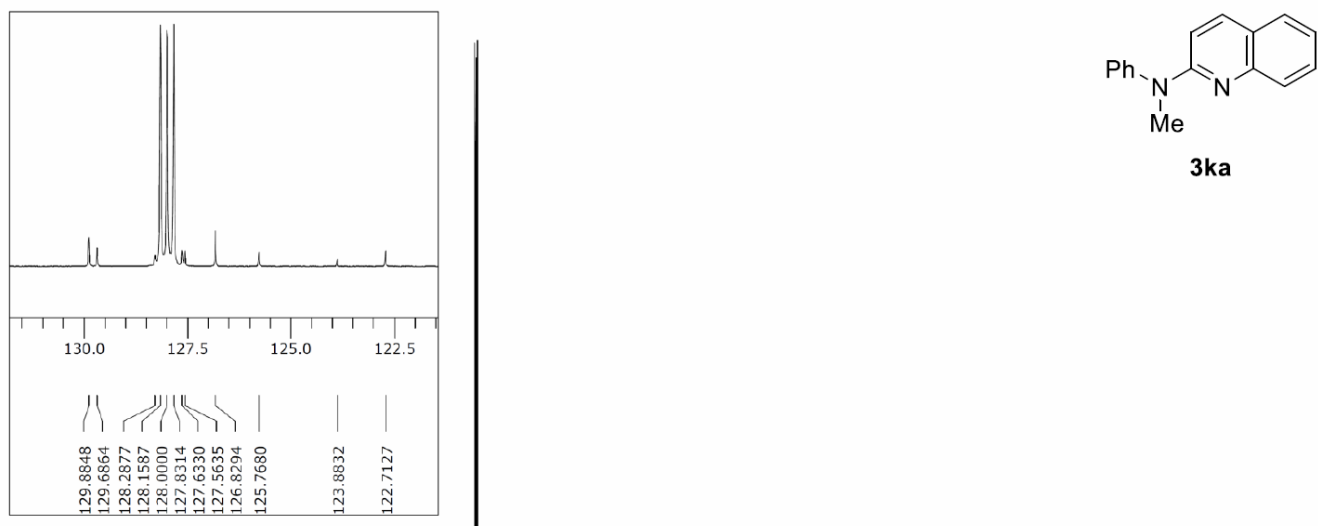

3ka

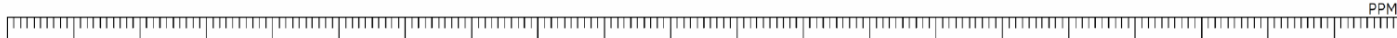
$\begin{array}{lllllllllllllllllllll}200.0 & 190.0 & 180.0 & 170.0 & 160.0 & 150.0 & 140.0 & 130.0 & 120.0 & 110.0 & 100.0 & 90.0 & 80.0 & 70.0 & 60.0 & 50.0 & 40.0 & 30.0 & 20.0 & 10.0 & 0.0\end{array}$

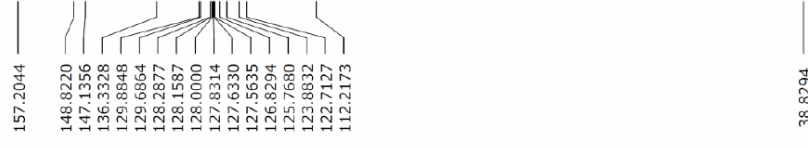


${ }^{1} \mathrm{H}$ NMR spectra of $\mathbf{3 l a}$

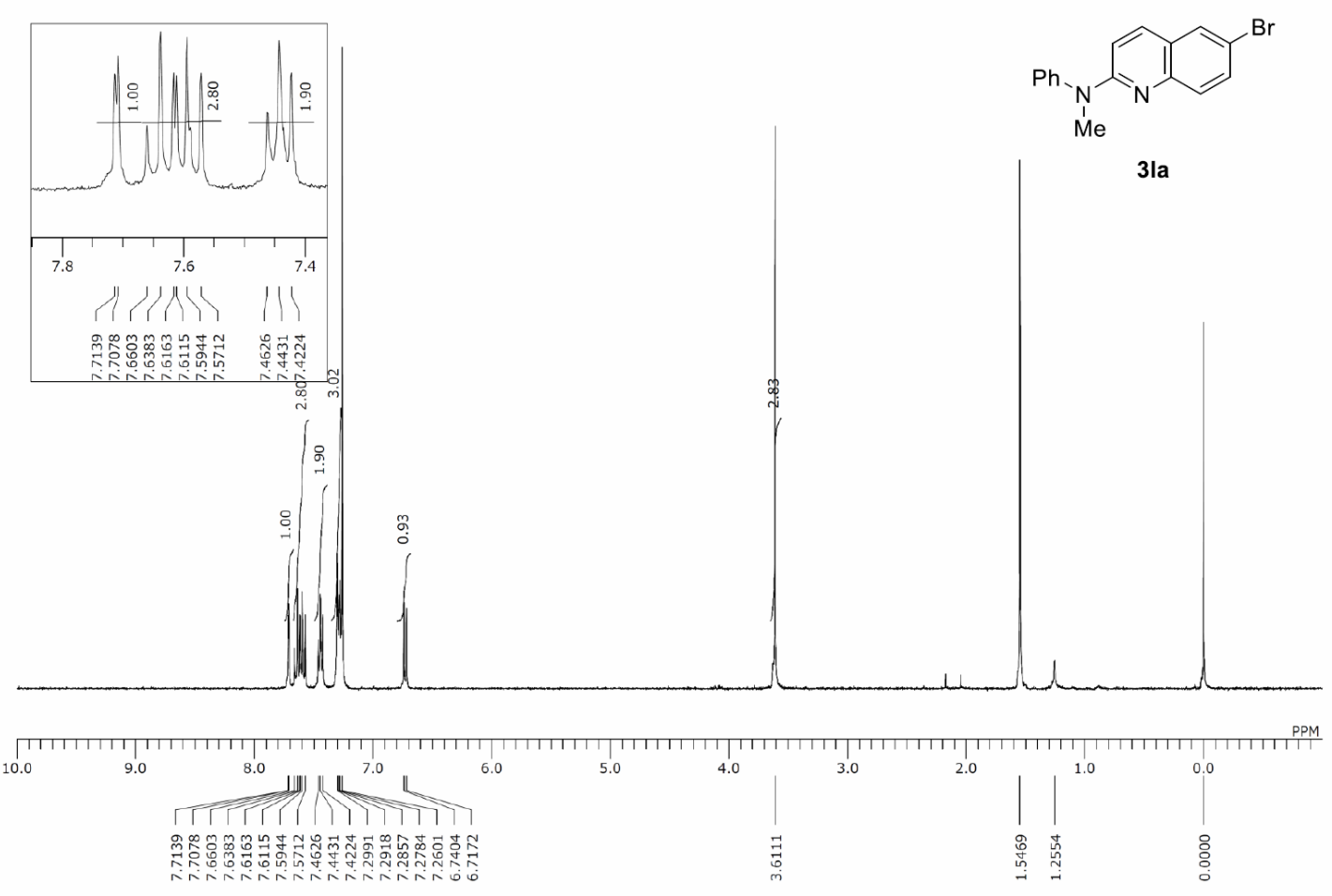

${ }^{13} \mathrm{C}$ NMR spectra of 3la

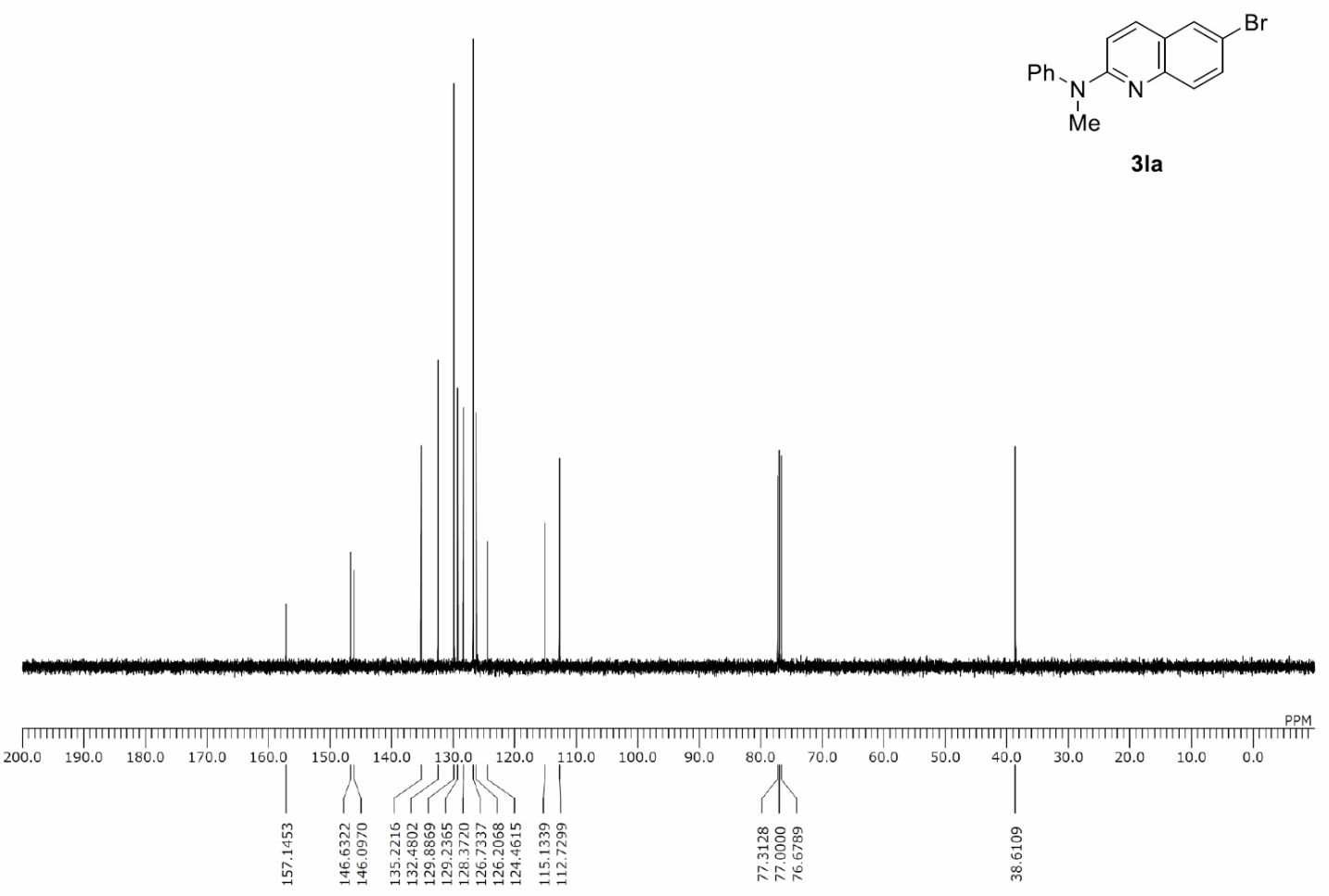



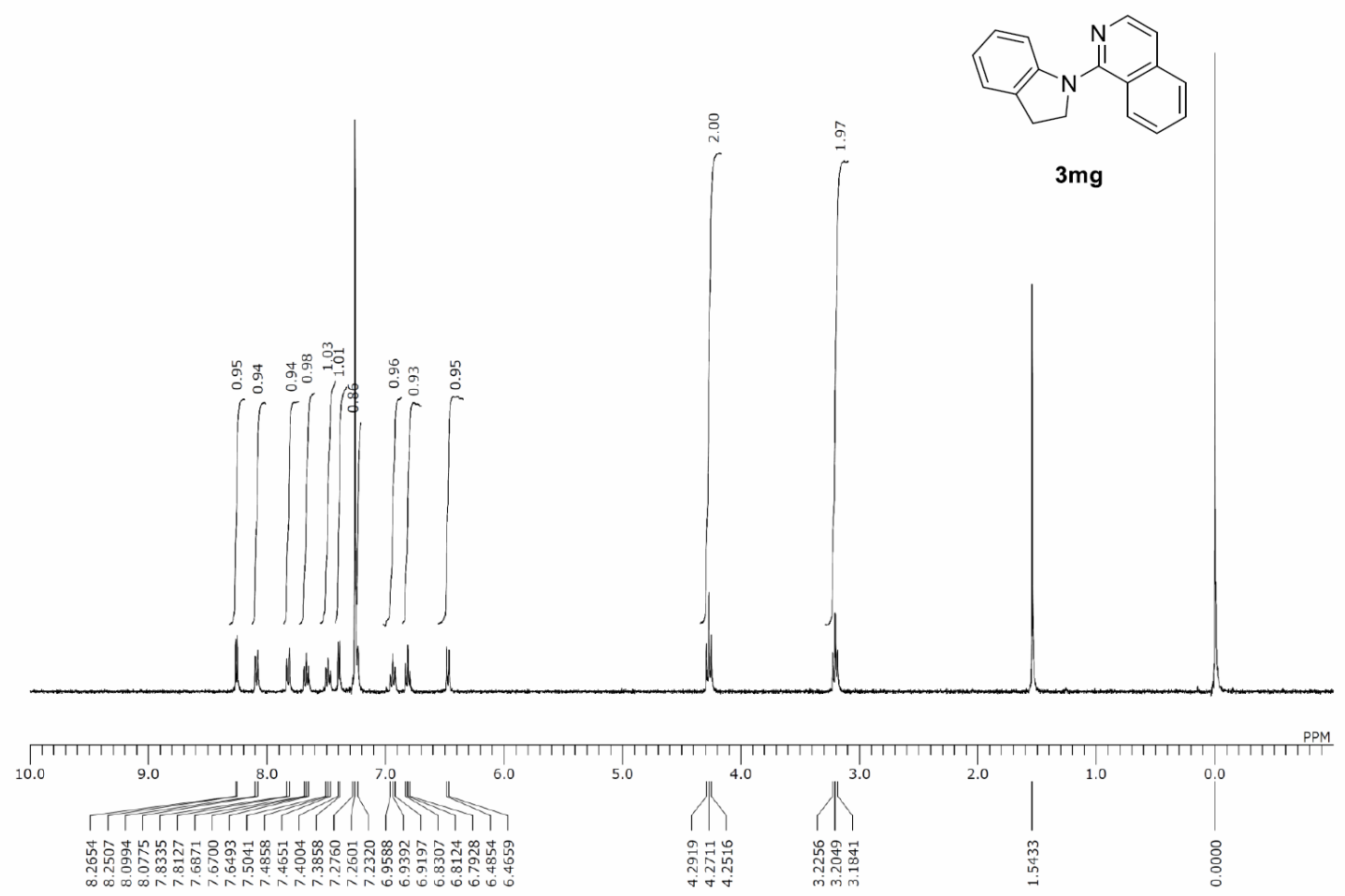

${ }^{13} \mathrm{C}$ NMR spectra of $\mathbf{3 m g}$
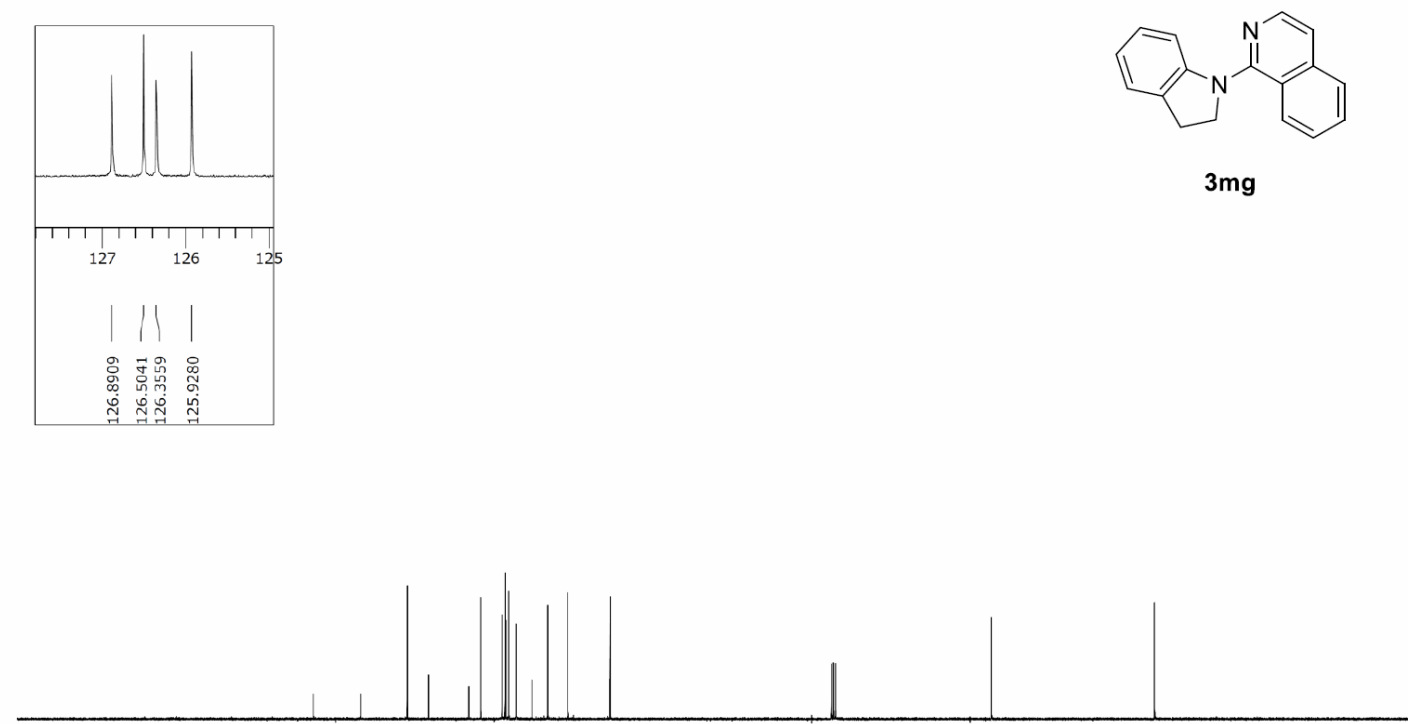

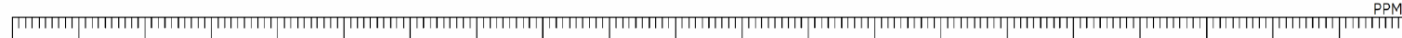
$\begin{array}{llllllllllllllllllllll}200.0 & 190.0 & 180.0 & 170.0 & 160.0 & 150.0 & 140.0 & 130.0 & 120.0 & 110.0 & 100.0 & 90.0 & 80.0 & 70.0 & 60.0 & 50.0 & 40.0 & 30.0 & 20.0 & 10.0 & 0.0 & \end{array}$

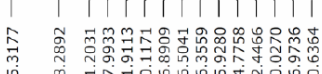

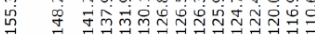

1

웅을

충 
${ }^{1} \mathrm{H}$ NMR spectra of $\mathbf{3 n a}$
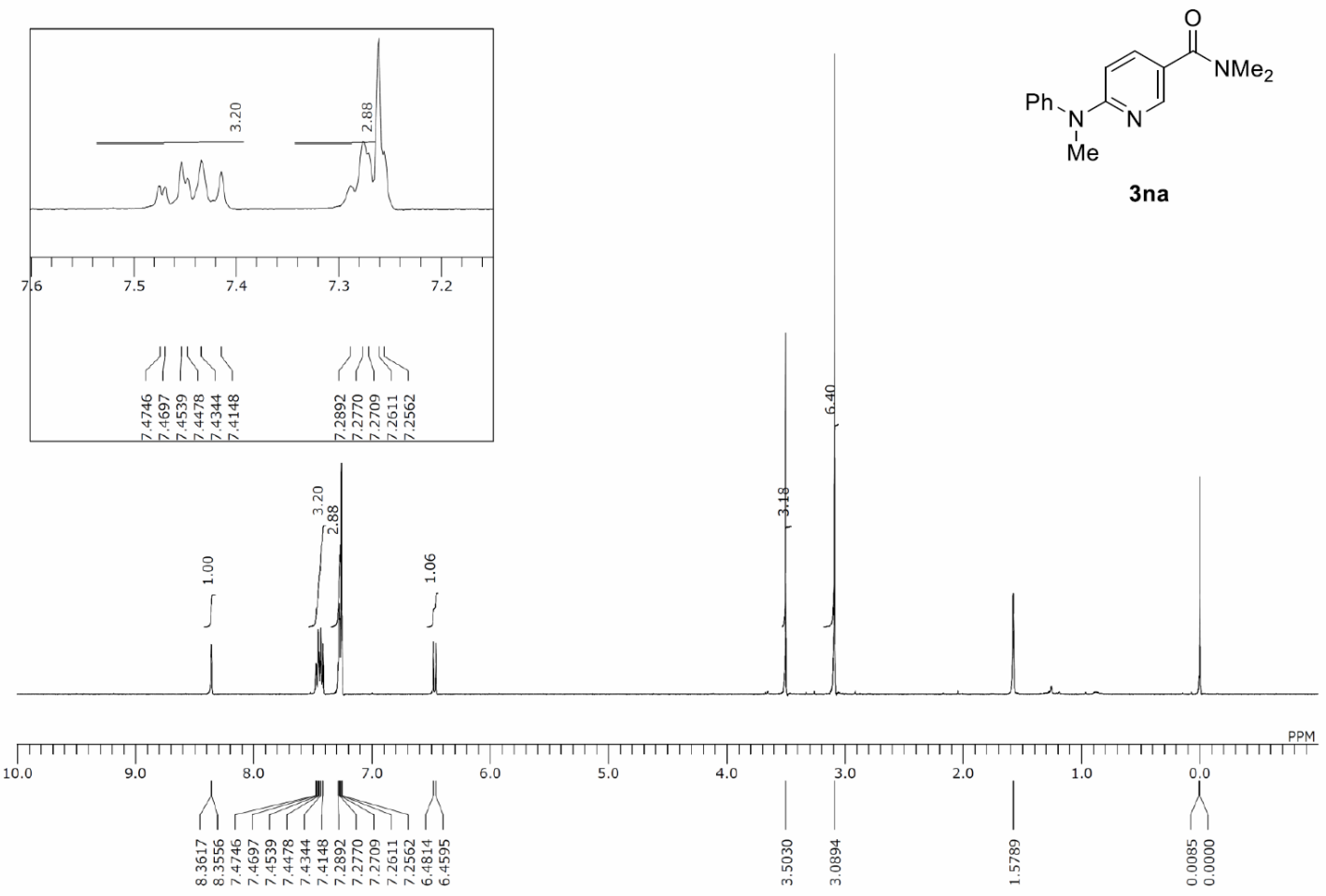

${ }^{13} \mathrm{C}$ NMR spectra of 3na

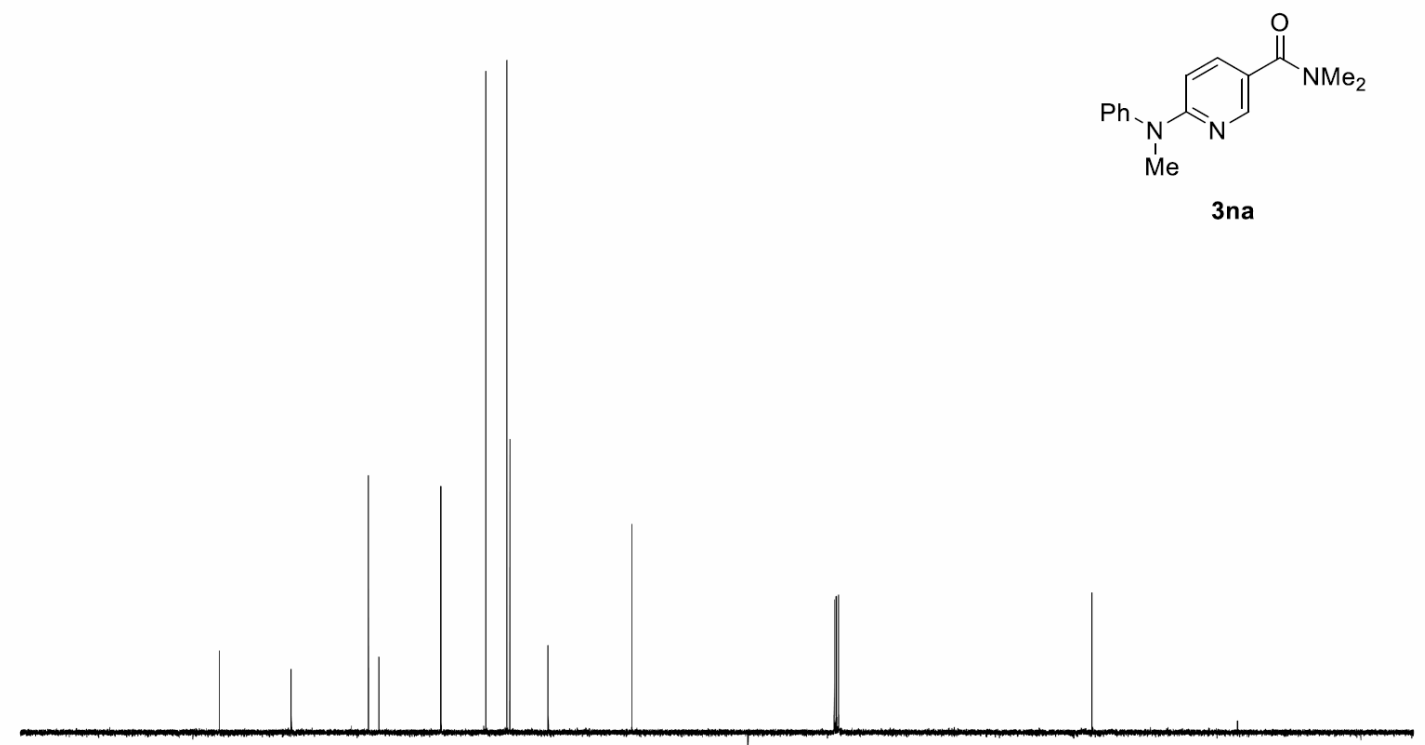

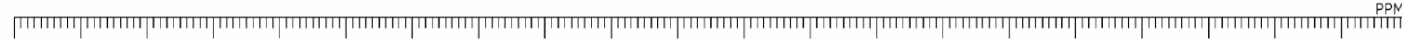

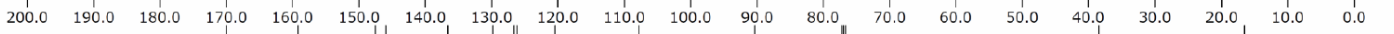

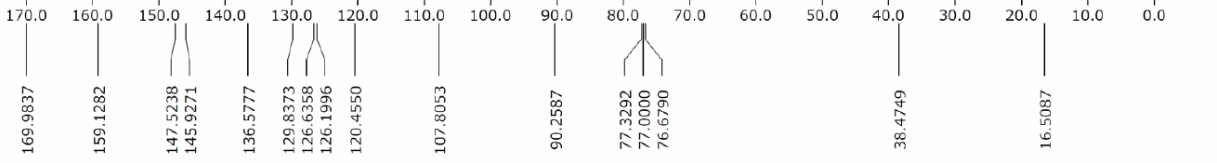




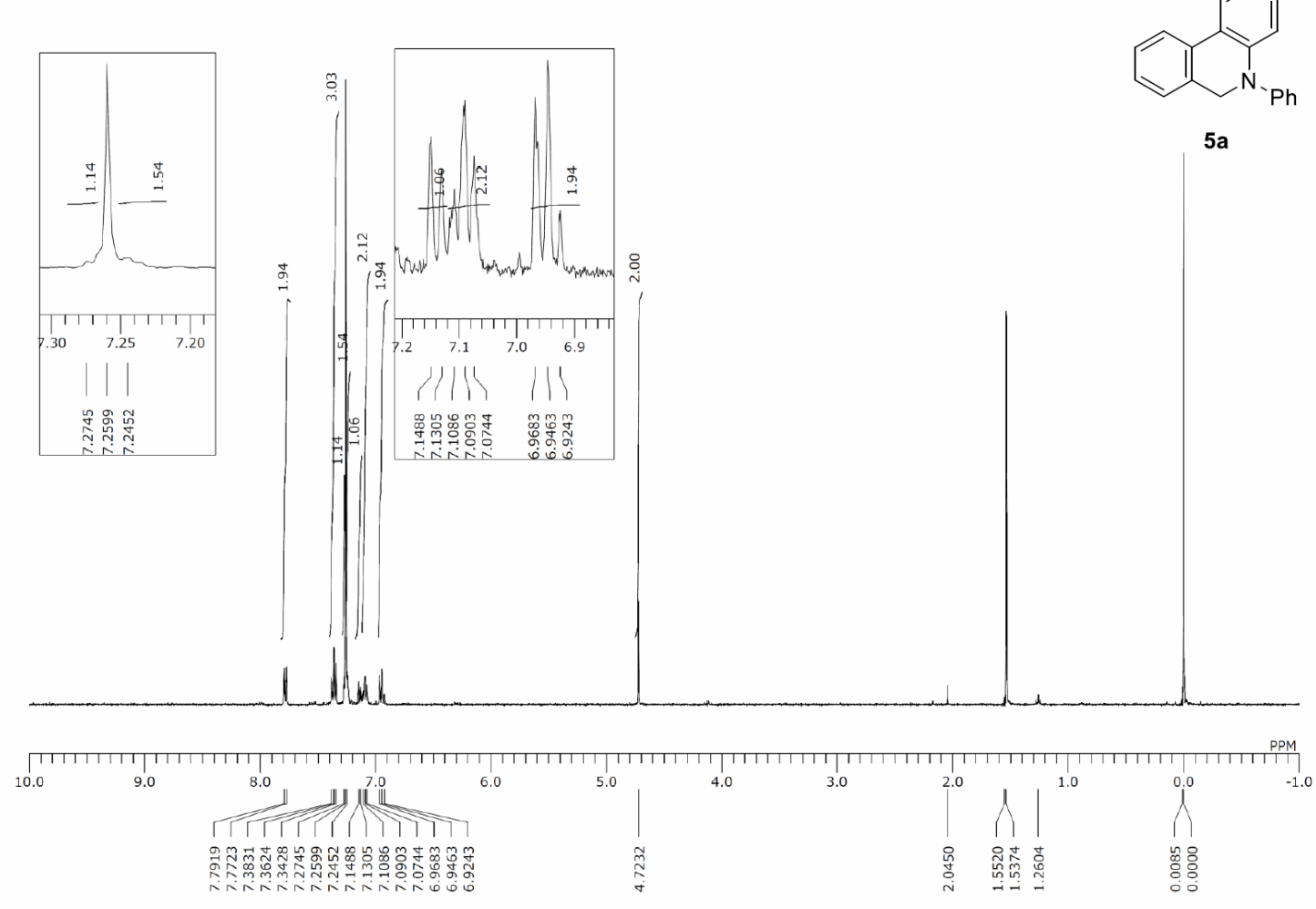

${ }^{13} \mathrm{C}$ NMR spectra of 5a

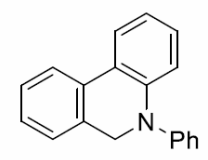

$5 a$

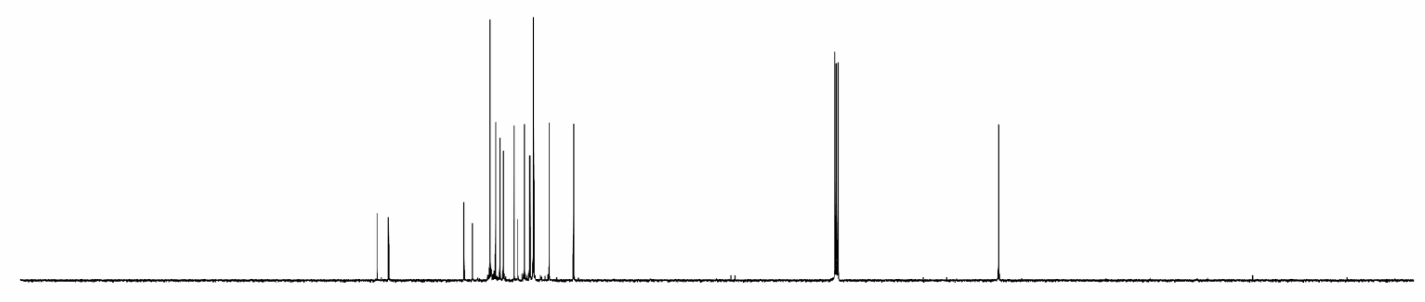

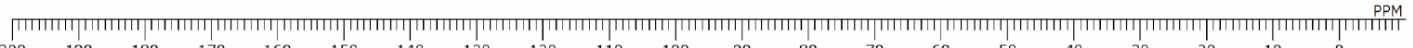
$\begin{array}{llllllllllllllllll}200 & 190 & 180 & 170 & 160 & 150 & 140 & 130 & 130 & 30 & 20 & 10 & 0\end{array}$

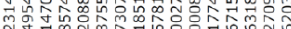

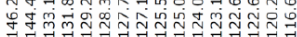

용응

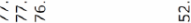



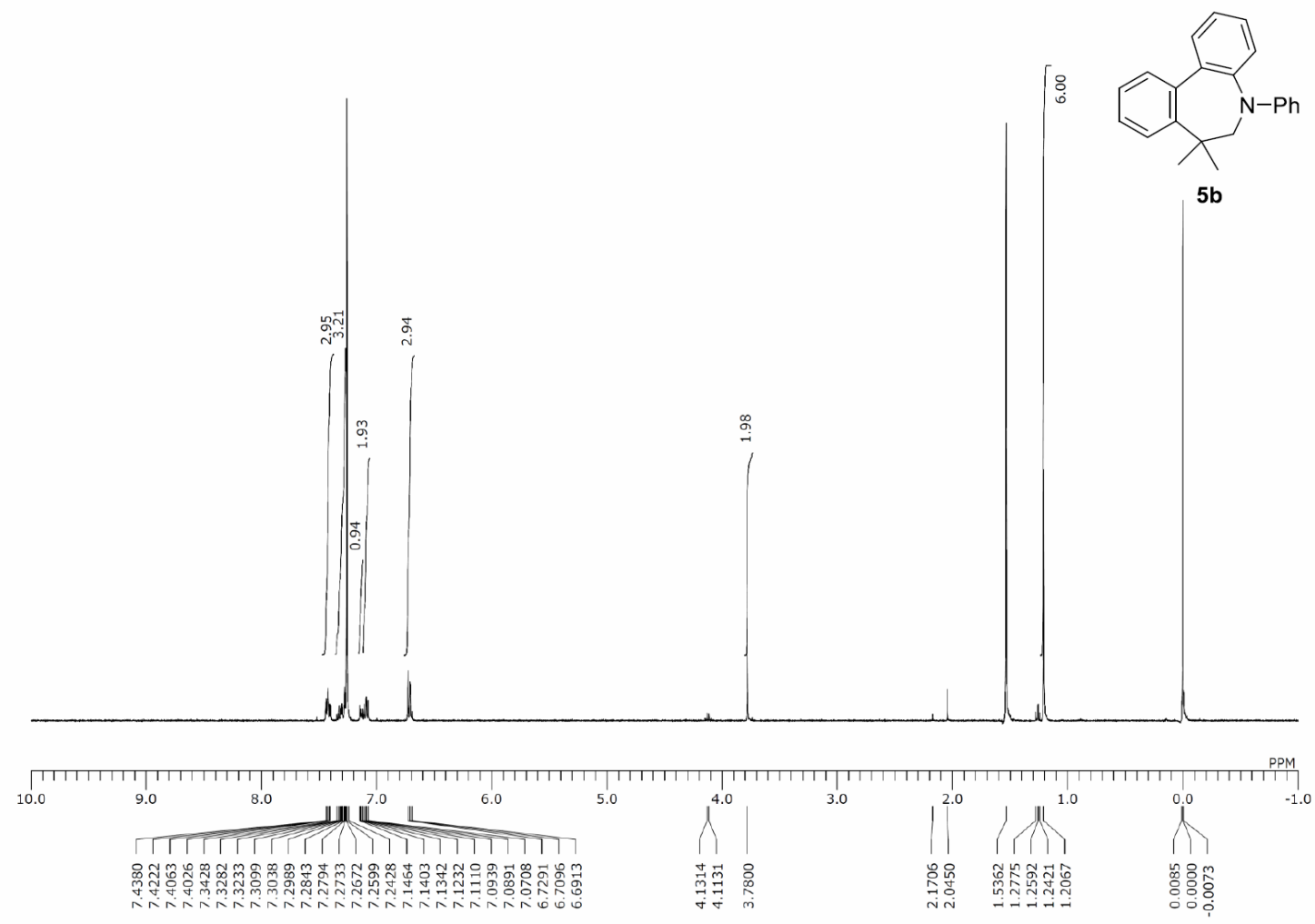

${ }^{13} \mathrm{C}$ NMR spectra of $\mathbf{5 b}$
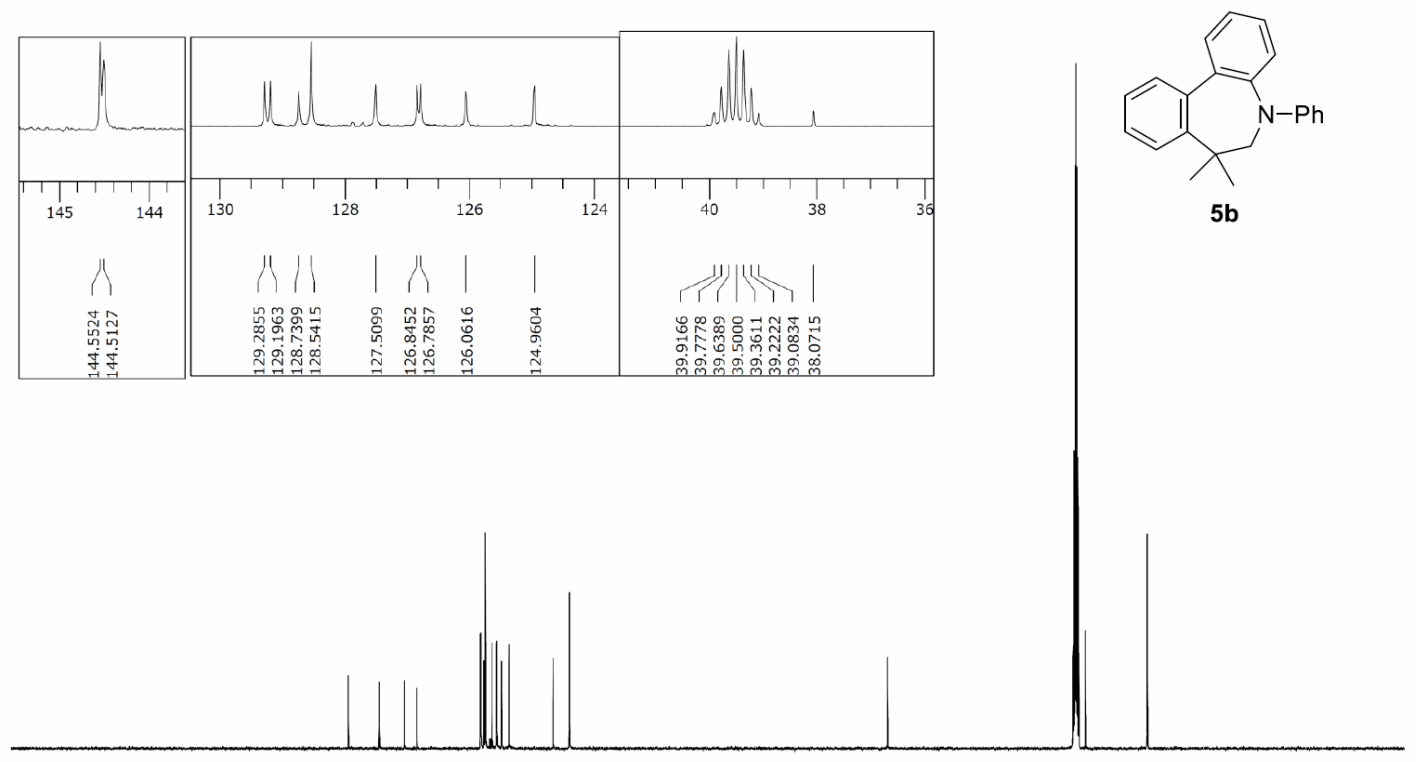

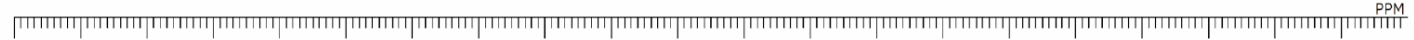

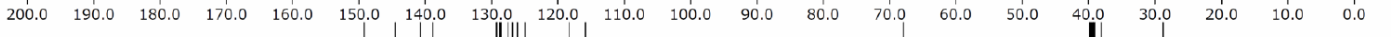

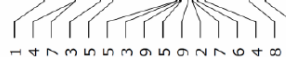

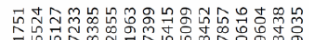

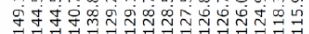

$\begin{array}{ll}7 & 0 \\ 0 & 0\end{array}$ 